\title{
A Unified Poisson-Cahn Methodology for Defect Segregation Near Grain Boundaries in Oxygen-Conducting Solid Electrolytes
}

Xiaorui Tong

Follow this and additional works at: https://researchrepository.wvu.edu/etd

\section{Recommended Citation}

Tong, Xiaorui, "A Unified Poisson-Cahn Methodology for Defect Segregation Near Grain Boundaries in Oxygen-Conducting Solid Electrolytes" (2018). Graduate Theses, Dissertations, and Problem Reports. 7267.

https://researchrepository.wvu.edu/etd/7267

This Dissertation is protected by copyright and/or related rights. It has been brought to you by the The Research Repository @ WVU with permission from the rights-holder(s). You are free to use this Dissertation in any way that is permitted by the copyright and related rights legislation that applies to your use. For other uses you must obtain permission from the rights-holder(s) directly, unless additional rights are indicated by a Creative Commons license in the record and/ or on the work itself. This Dissertation has been accepted for inclusion in WVU Graduate Theses, Dissertations, and Problem Reports collection by an authorized administrator of The Research Repository @ WVU.

For more information, please contact researchrepository@mail.wvu.edu. 


\title{
A Unified Poisson-Cahn Methodology for Defect Segregation Near Grain Boundaries in Oxygen-Conducting Solid Electrolytes
}

\author{
Xiaorui Tong \\ Dissertation submitted \\ to the Benjamin M. Statler College of Engineering and Mineral Resources \\ at West Virginia University
}

in partial fulfillment of the requirements for the degree of

Doctor of Philosophy

in

Material Science and Engineering

David S. Mebane, Ph.D., Chair

Edward M. Sabolsky, Ph.D.

John A. Christian, Ph.D.

Harry Abernathy, Ph.D.

Fernando V. Lima, Ph.D.

Department of Mechanical and Aerospace Engineering

Morgantown, West Virginia

2018

Keywords: Oxygen Ion Conductor, Grain Boundary, Space Charge, Dopant Segregation Copyright 2018 Xiaorui Tong 


\section{ABSTRACT \\ A Unified Poisson-Cahn Methodology for Defect Segregation Near Grain Boundaries in Oxygen-Conducting Solid Electrolytes}

\section{Xiaorui Tong}

In the Poisson-Cahn theory, the incorporation of defect interactions and the gradient effects has made it possible to model interfaces in solid solutions of high concentrations. This study aimed at building a unified Poisson-Cahn methodology capable of conforming to physically reasonable parameter spaces and incorporating potentially complex concentrationdependences of model parameters to enable broader future applications in various material systems.

The first development established a kinetic model to predict defect segregation during thermal annealing of these systems. The complete process of dopant segregation from the initial flat distribution all the way to the equilibrium was firstly revealed, and the amount of equilibration time for dopant ions was obtained at different temperatures. Secondly, the Poisson-Cahn theory was applied to a two-step restricted equilibrium model in estimating the conductivity behavior of gadolinium-doped-ceria over a broad range of dopant concentrations. The consistency between the model predicted conductivity data and experimental measurement has demonstrated the effectiveness of this scheme.

In order to specify parameter ranges with higher confidence and to gauge potential parameter dependences on defect concentrations, it is necessary to calibrate model parameters with direct grain boundary measurements. We fit the atom probe data of Nd-doped ceria with $10 \%$ and $30 \%$ dopant respectively to a restricted Poisson-Cahn equilibrium model with added polaron species but found the values for the gradient energy coefficients weren't in agreement with what are reported in literature. This led to the formulation and implementation of a data-driven approach.

Within the framework of a unified Poisson-Cahn methodology, a series of models are then designed through systematically incorporating different interaction energies and gra- 
dient energy effects in the form of discrepancy functions. Bayesian calibration is employed for each model using grain boundary compositions of Ca-doped ceria with $2 \%, 5 \%$ and $10 \%$ dopant concentrations. The development of this model framework has shown how the model capability improves by including key model parameters and revealed the concentration dependences of model parameters through quantitatively evaluating the calibration results. An optimal model structure was obtained with the guidance of the Bayes factor. 


\section{ACKNOWLEDGEMENTS}

First and foremost, I would like to express my sincere appreciation and gratitude to my research advisor - Dr. David Mebane, for his full support during my Ph.D study at WVU. He introduced me into a new research area and guided me towards the goal of becoming a computational material scientist with the utmost encouragement, intellectual guidance and inspirational resources. This work is impossible without him and I couldn't imagine having a more supportive advisor.

My sincere thanks and appreciation also goes to the rest of my committee members. Thanks for serving on my Ph.D committee and bearing with me on the logistics. Thanks to all your constructive suggestions and inspirational questions. They have greatly helped me improve my research and become more professional.

I would like to thank Dr. Roger De Souza at RWTH Aachen University for his kind guidance, constructive suggestions and fruitful collaborations on publications. I would also like thank his student Alex Zurhelle, for the summer working together to improve the capability of the Poisson-Cahn model. Thank Dr. David Diercks at Colorado School of Mines for providing the Atom Probe data. Thank Dr. William Bowman at MIT and Dr. Peter Crozier at Arizona State University for the EELS data. These have been extremely helpful for me in model development.

I also want to thank all my lab colleagues at the Energy Systems and Materials Simulation Group. Thank Kuijun Li, for sharing his study experiences on Bayesian calibration with discrepancy functions. Thank Keenan Kocan and Alejandro Mejia for extra help with proofreading my proposal. Thank all of them including Guiseppe Brunello, Brian Logston and Ahmed Nirjhar for the intellectual discussions, knowledge sharing, group meeting talks and all the good times spent together.

Thank all my friends who have graduated and who are continuing their study here at WVU. With all of you here, life is so much more fun. Thanks for sharing all the experiences, for being willing to listen, and for enriching life in Morgantown. 
Last but not the least, I couldn't express enough gratitude to my mother Hongmei, who have provided unconditional support to make things possible. She made sacrifices to help us take care of our baby during the busiest time of my study so that I can finish developing the unified Poisson-Cahn model. Thanks for showing me the greatest love of a mum with actions. I would also love to thank my grandparents for their endless love.

I also want to thank my husband Peng. He is the reason that brought me here. This college town has witnessed our marriage and the birth of our baby Emma. Life with them is blessed with happiness.

Thank God for all the arrangements and for guiding me to grow. 


\section{TABLE OF CONTENTS}

Page

1. INTRODUCTION . . . . . . . . . . . . . . . . . . . . . 1

2. LITERATURE BACKGROUND . . . . . . . . . . . . . . . . . . 5

2.1 Experimental Findings on Grain Boundaries in Solid Electrolyte....... . 5

2.1.1 Microstructure and Composition ................ 5

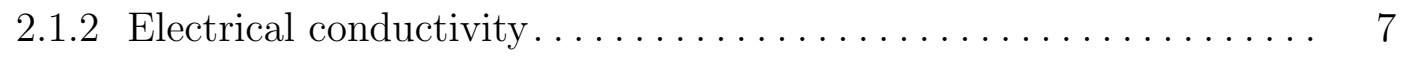

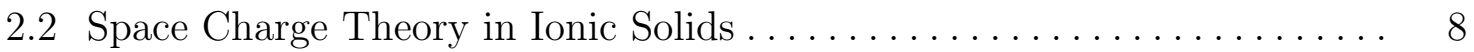

2.2 .1 Gouy-Chapman............................. 10

2.2 .2 Mott-Schottky ......................... 11

2.3 Gap between Experimental Observations and Space Charge Theories... . 13

2.4 Variational Phase Field Models . . . . . . . . . . . . . . . . . . 15

2.5 Nakayama-Martin Oxygen ion conductivity model . . . . . . . . . . 17

2.6 Bayesian Framework for Model Parameterization . . . . . . . . . . . . . . 19

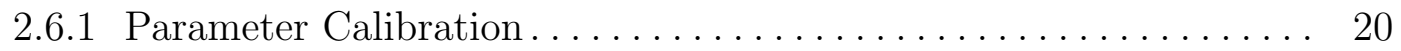

2.6 .2 Gaussian Processes.......................... 22

2.6 .3 The BSS-ANOVA Framework $\ldots \ldots \ldots \ldots \ldots \ldots \ldots \ldots \ldots \ldots$

3. A GENERALIZED SPACE CHARGE THEORY: POISSON-CAHN . 26

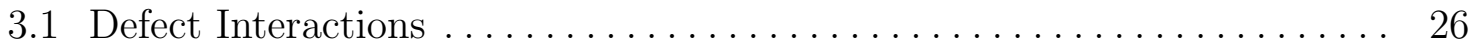

3.2 Gradient Energy Contribution $\ldots \ldots \ldots \ldots \ldots \ldots \ldots \ldots \ldots \ldots \ldots \ldots \ldots \ldots \ldots \ldots \ldots$

3.3 Derivation of the Poisson-Cahn Model . . . . . . . . . . . . . . 31 
4. KINETIC MODELING FOR DOPANT SEGREGATION . . . . . . 35

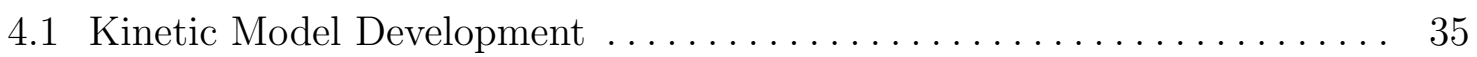

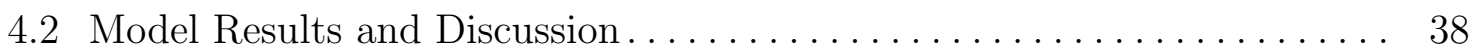

5. POISSON-CAHN MODEL PARAMETERIZATION . . . . . . . . 45

5.1 Modeling Grain Boundary Resistance of GDC . . . . . . . . . . . . 45

5.2 Modeling Grain Boundary Composition in NDC . . . . . . . . . . . . . 48

6. DEVELOPING A UNIFIED POISSON CAHN FRAMEWORK WITH BSS-ANOVA METHOD . . . . . . . . . . . . . . . . . 60

6.1 Derivation of a Unified Poisson-Cahn Framework using BSS-ANOVA Dis-

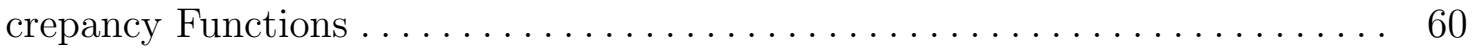

6.2 Systematic Model Building with Bayesian Calibration. . . . . . . . . . . 69

6.3 Model Comparison Using the Bayes Factor $\ldots \ldots \ldots \ldots \ldots \ldots \ldots \ldots \ldots$

7. CONCLUSIONS .................... 90

REFERENCES. . . . . . . . . . . . . . . . . . . . . . . . . 94 


\section{LIST OF TABLES}

Table 4.1 Model parameter values $\ldots \ldots \ldots \ldots \ldots \ldots \ldots \ldots \ldots \ldots \ldots \ldots \ldots$

Table 5.1 Fitted parameter values from particle swarm optimization . . . . . . . 46

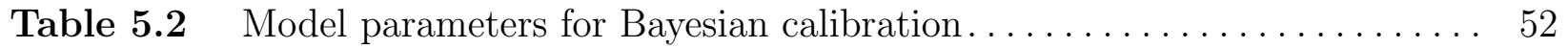

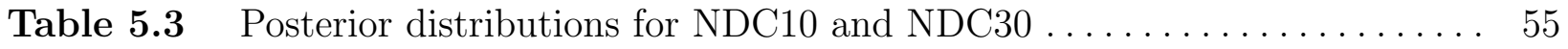

Table 6.1 Design of Model structures through Systematic Building. . . . . . . . . 70

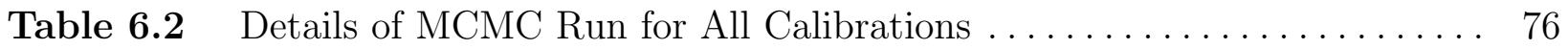

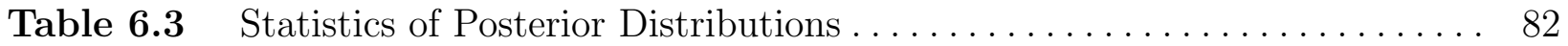




\section{LIST OF FIGURES}

Figure 2.1 Example set of basis functions from $[1] \ldots \ldots \ldots \ldots \ldots \ldots \ldots \ldots \ldots \ldots$

Figure 4.1 Dopant profile as a function of distance from the interface at $1300{ }^{\circ} \mathrm{C}$ and $20 \%$ dopant cation site fraction, during the initial $10^{-7} \mathrm{~s}$ of

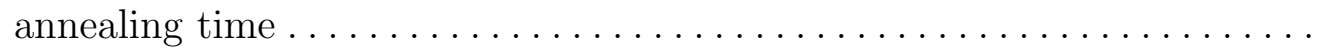

Figure 4.2 Dopant profile as a function of distance from the interface at $1300{ }^{\circ} \mathrm{C}$ and $20 \%$ dopant cation site fraction, during the initial $10^{-7} \mathrm{~s}$ of annealing time (zoomed in to show the second segregation zone)...... 40

Figure 4.3 Dopant profile as a function of distance from the interface at $1300{ }^{\circ} \mathrm{C}$ and $20 \%$ dopant cation site fraction, after the second segregation zone begins to disappear ............................... 40

Figure 4.4 Dopant profile as a function of distance from the interface at $1300{ }^{\circ} \mathrm{C}$ and $20 \%$ dopant cation site fraction, between $10^{-7}$ and $10^{-5} \mathrm{~s} \ldots \ldots \ldots 41$

Figure 4.5 Dopant profile as a function of distance from the interface at $1300{ }^{\circ} \mathrm{C}$ and $20 \%$ dopant cation site fraction, at $\mathrm{t}=0.001,0.0015$ and $0.017 \mathrm{~s} \ldots . .42$

Figure 4.6 Dopant profile as a function of distance from the interface at $1300{ }^{\circ} \mathrm{C}$ and $20 \%$ dopant cation site fraction, at $\mathrm{t}=0.03,0.07,0.1 \mathrm{~s}$ and at equilibrium .......................................

Figure 4.7 Vacancy site fraction as a function of distance from the interface at $1300{ }^{\circ} \mathrm{C}$ and $20 \%$ dopant cation site fraction 
Figure 4.8 Electrostatic potential as a function of distance from the interface at $1300{ }^{\circ} \mathrm{C}$ and $20 \%$ dopant cation site fraction $\ldots \ldots \ldots \ldots \ldots \ldots \ldots$

Figure 4.9 Time to equilibrium as a function of annealing temperature for GDC with $20 \%$ dopant concentration. .................. 44

Figure 5.1 Conductivity $\sigma$ of the $\mathrm{CeO}_{2}-\mathrm{Gd}_{2} \mathrm{O}_{3}$ system as a function of $n_{a}$ at

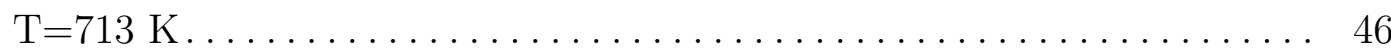

Figure 5.2 Dopant concentration profiles, $n_{a}(\mathrm{x})$, calculated from Poisson-Cahn theory(red) and from Gouy-Chapman theory(black) for six values dopant concentrations at $\mathrm{T}=1223 \mathrm{~K}$.

Figure 5.3 Extent of space-charge layer calculated from Poisson-Cahn theory. Compared with $3.5 l_{D}$. Extracted from $\varphi(x)=0.0078 \varphi_{0} \ldots \ldots \ldots \ldots .48$

Figure 5.4 Raw APT data for $10 \%$ (left) and $30 \%$ samples $\ldots \ldots \ldots \ldots \ldots \ldots$

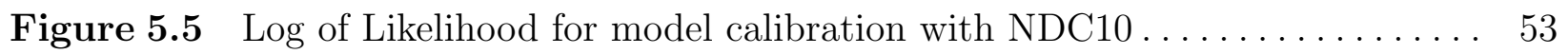

Figure 5.6 Selected parameters vs. MCMC steps for calibration of NDC10 . . . . 53

Figure 5.7 Selected parameters vs. MCMC steps for calibration of NDC30 _ . . . 54

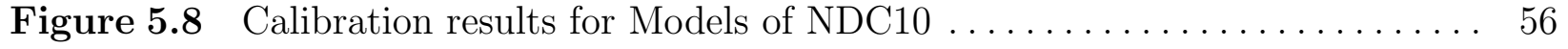

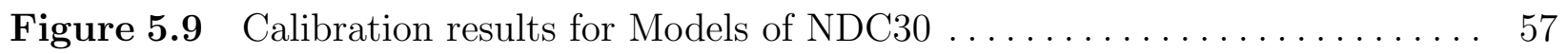

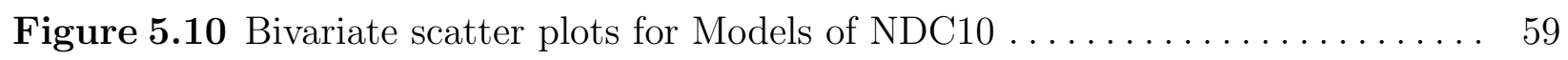

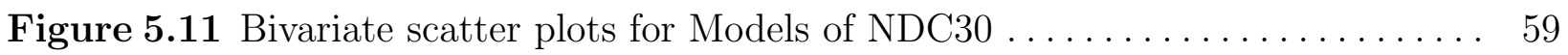

Figure 6.1 Experimental data of $\mathrm{CCO} 2[2] \ldots \ldots \ldots \ldots \ldots \ldots \ldots \ldots \ldots \ldots \ldots$ 
Figure 6.2 Experimental data of CCO5 $[2] \ldots \ldots \ldots \ldots \ldots \ldots \ldots \ldots \ldots \ldots \ldots \ldots \ldots$

Figure 6.3 Experimental data of $\mathrm{CCO} 10[2] \ldots \ldots \ldots \ldots \ldots \ldots \ldots \ldots \ldots \ldots \ldots$

Figure 6.4 Calibration Results for Baseline Model V0 .................... 77

Figure 6.5 Calibration Results for Model V7 ...................... 78

Figure 6.6 Calibration Results for Model V8 ......................... 79

Figure 6.7 Calibration Results for Model V19...................... 80

Figure 6.8 Calibration Results for Model V20...................... 81

Figure 6.9 Model Performance Quantified by the Bayes Factor ................ 84

Figure 6.10 Function Components of $f_{y}$ and $f_{v} \ldots \ldots \ldots \ldots \ldots \ldots \ldots \ldots$

Figure 6.11 Function form of $f_{y}$ and $f_{v} \ldots \ldots \ldots \ldots \ldots \ldots \ldots \ldots \ldots \ldots \ldots$

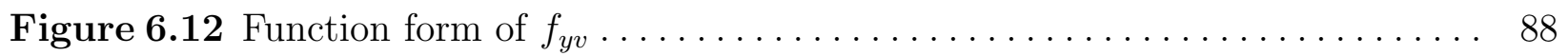

Figure 6.13 Vacancy Profiles near the GB as Evaluated with Model V19 ......... 89 


\section{CHAPTER 1}

\section{INTRODUCTION}

With the ever increasing energy demand and the limited resources of fossil fuels, efficient energy sources have long been a focused area of research. At the same time, it is now well known that global warming is continuing unabated due to effluent gas emission, mainly $\mathrm{CO}_{2}$ [3]. According to the Goddard Institute for Space Studies (GISS) analysis of global surface temperature change, the annual mean land-ocean temperature has increased from $-0.3^{\circ} \mathrm{C}$ in 1910 to near $0.6^{\circ} \mathrm{C}$ in 2010 [4] and this trend has dramatically increased during the past decades. Issues associated with energy supply and uses are related not only to global warming, but also to other environmental concerns such as air pollution, acid precipitation, ozone depletion, forest destruction, and emission of radioactive substances. Therefore, these energy and environment related challenges call for the generation of energy by clean, efficient and environmental-friendly means.

Solid Oxide Fuel Cells (SOFCs) have attracted much attention as promising candidates for future energy solutions owing to their high efficiency, low environmental impact, fuel flexibility and low cost. A SOFC is an all-solid-state energy conversion device that converts the chemical energy of a fuel gas directly to electrical energy without the need for combustion. It consists of two electrodes sandwiched around a hard ceramic electrolyte such as yttrium-stabilized-zirconia (YSZ) or gadolinium doped ceria (GDC) etc. Gaseous fuel, such as $\mathrm{H}_{2}, \mathrm{CH}_{4}$ and $\mathrm{CO}$, is fed into the anode of SOFC and oxygen from the air enters the cell through cathode. Through the fuel reaction with oxygen ions at the anode side, the concentration of oxygen is dramatically reduced. Since the cathode is exposed to air, an oxygen concentration gradient is thus created across the electrolyte, attracting oxygen ions from the cathode side to the anode side. Providing an electrical connection between 
the cathode and the anode, electrons will flow from the anode to the cathode. As long as a continuous supply of oxygen ions for the electrolyte is maintained, electrical power will be generated through oxidation of the fuel gas. The reactions at both electrodes are expressed below(taking $\mathrm{H}_{2}$ as the fuel):

$$
\begin{aligned}
& \text { Anode }: \mathrm{H}_{2}+\mathrm{O}_{\mathrm{O}}^{\mathrm{x}} \longrightarrow \mathrm{H}_{2} \mathrm{O}+2 \mathrm{e}^{-}+\mathrm{V}_{\mathrm{O}} \\
& \text { Cathode }: \mathrm{O}_{2}+4 \mathrm{e}^{-}+2 \mathrm{~V}_{\ddot{O}} \longrightarrow 2 \mathrm{O}_{\mathrm{O}}^{\mathrm{x}}
\end{aligned}
$$

where $\mathrm{V}_{\mathrm{O}} \ddot{\text { and }} \mathrm{O}_{\mathrm{O}}^{\mathrm{x}}$ represent doubly charged oxygen vacancies and oxygen ions at the normal lattice sites in the crystal structure, respectively. The solid electrolyte is the central part of a SOFC that allows the flow of oxygen ions from the cathode to the anode to maintain the overall charge balance. Its ability to conduct oxygen ions and block electrons is critical for the overall performance of the SOFC system. Oxygen ion conducting electrolytes cover a wide range of materials which basically include fluorite-, perovskite, brownmilleritestructured materials. Fluorite-structured materials, a representative system is rare-earth doped ceria, have drawn much attention during the past decades since the discovery of their high ionic conductivity under intermediate temperature $\left(500-750{ }^{\circ} \mathrm{C}\right)$. In response to the demand of reducing the operating temperature of SOFC for better thermal management and easier maintenance, intermediate temperature solid oxide fuel cell (IT-SOFC) has become a favorable choice and more research is therefore focused on oxygen conducting solid electrolytes that exhibit high ionic conductivity in this temperature regime.

Cerium oxide, when doped with acceptor cations, exhibits high ionic conductivity due to extrinsic oxygen vacancies, which are generated for charge compensation. On the other hand, the loss of oxygen to the gas phase can result in the increase of electronic conductivity, making ceria a mixed ionic-electronic conductor (MIEC). While the mixing of electronic and ionic conductivity may be desirable for certain applications (e.g. oxygen membranes, electrodes), the use of cerium oxide as solid electrolyte requires that the electronic contribution 
being negligible compared with the ionic partial conductivity.

Research evidence has shown that the existence of interfaces, e.g. grain boundaries, are key factors that affect the ionic conductivity of these materials possibly due to the formation of second phases caused by impurity silicon $[5,6]$, and dopant segregation and oxygen vacancy depletion near the grain boundary core $[7,8]$. For materials of high purity, the space charge effect at the grain boundaries becomes the dominating factor [9, 10]. Solute segregation and the formation of space charge zones near the interfaces of solid electrolytes exert a significant effect on the conductivity as well as other properties of these materials, resulted in deleterious consequences in real-world applications. Researchers have been studying the mechanisms behind the formation of space charge zones and theories have been proposed to model this phenomenon. With the advancement of microscopy techniques, experimentalists have been able to probe fine details of the interface structures and reveal information that was once unavailable. These observations have further facilitated the development of space charge theories and thus manifested flaws of certain assumptions adopted in the current space charge model, as certain experimentally observed evidence can not be predicted by the current theory.

Continuum space-charge models in Gouy-Chapman or Mott-Schottky approximations can be employed in dilute systems to estimate defect profiles near GBs [8]. For highly-doped systems the application of these models becomes problematic, as segregation layers extending several nm where the Debye length is less than an angstrom [11], cannot be predicted. It is the purpose of this Ph.D. study to model defect segregation near grain boundaries for oxygen-conducting solid electrolytes across the concentration range, searching for a uniform theory that can predict defect behaviors in both dilute and concentrated systems.

A generalized space-charge theory, based on the Cahn-Hilliard theory for the thermodynamics of solute segregation in alloys, has been developed to incorporate defect interactions and effects of gradient energy which become significant in concentrated systems $[12,13]$. This "Poisson-Cahn" approach has been previously employed in a surface model 
for mixed ionic-electronic conductors (MIEC) [13], and in predicting the ionic conductivity of the $\mathrm{CeO}_{2}-\mathrm{Gd}_{2} \mathrm{O}_{3}$ at $440{ }^{\circ} \mathrm{C}$. The main contribution of this theory is that by including defect interactions and the gradient energy effects, it can be readily applicable to model defect re-distribution near interfaces over the entire concentration range, from dilutely doped systems to solid solutions.

The main mission of this research is to build a unified methodology based on the Poisson-Cahn theory in order to model defect profiles near the interface within a consistent model parameter space for doped materials of any dopant concentration. Knowledge from these developments will establish a theoretical pathway to effectively model the interface effects in oxygen-ion conducting solid electrolytes and thus contribute to tailoring current material structures and proposing new material systems. For broader applications, this methodology can be employed to treat a variety of solid ionic systems, not limited to oxygenion conductors. 


\section{CHAPTER 2}

\section{LITERATURE BACKGROUND}

\subsection{Experimental Findings on Grain Boundaries in Solid Electrolyte}

With the rapid technical advancement in solid oxide fuel cells, oxygen-ion conducting solid electrolyte has attracted significant research interest. Interfaces, such as surfaces, grain boundaries, sensitively affects multiple macroscopic properties of these materials, including the electrical property, mechanical property and optical properties etc. Therefore, many theoretical and experimental studies have been done on the effect of interfaces in solid electrolytes, especially on the grain boundary effect. Fluorite-structured ceramics are excellent candidates for solid electrolyte due to the long-term stability and high ionic conductivity under elevated temperatures. One material system that has been widely employed is $\mathrm{CeO}_{2}-\mathrm{M}_{2} \mathrm{O}_{3}$. The total conductivity of doped ceria is sufficiently high, making it suitable for applications as oxygen-ion conducting electrolytes in intermediate temperature solid oxide fuel cells (IT-SOFC). One technological limitation that comes along is that cost-effective production result in polycrystalline materials. The resistivity of these materials are there-

fore largely affected by the extensive existence of grain boundaries. This section reviews the state-of-the-art on grain boundaries in fluorite-structured ceramics in terms of grain boundary microstructure, composition and electrical conductivity.

\subsubsection{Microstructure and Composition}

In polycrystalline samples of fluorite-structured solid electrolytes, silicon based second phases separating individual grains were observed, which largely hinders the ionic conductivity of grain boundaries.

Using highly pure precursors can result in single-phase structure and thus largely 
reduce this negative effect. Thermogravimetry/differential thermal analysis (TG/DTA) and XRD have been used to study the calcination results of CGO and indicated a single-phase fluorite structure. TEM characterization revealed GDC samples exhibit a clean microstructure with grain boundaries free of detectable glassy phases. However, low GB conductivity still remains an issue upon the removal of second phases. This demands a further study of the grain boundary effect of materials with high purity. Investigating the composition near GB is therefore necessary.

Over the past several decades, dopant segregation has been widely observed/deduced through various experiments. Scanlon et al. used Low Energy Ion Scattering (LEIS) to determine the atomic composition of the outermost layers of CGO and found the near surface layers to be heavily Gd enriched [14]. This Gd enrichment decreased from $\mathrm{Ce} / \mathrm{Gd}=1$ to the bulk value of $\mathrm{Ce} / \mathrm{Gd}=4.2$ over five monolayers. Energy-loss spectra of GDC from a scanning transmission electron microscope (STEM) showed a marked increase in the Gd edge integrated intensity ratio at the GB core, indicating Gd concentration enhancement at the GB [15]. Lei et al. [16] confirmed Gd-segregation using a combination of Z-contrast imaging and electron energy-loss spectroscopy (EELS) and the width of the segregation region was measured to be approximately $2 \mathrm{~nm}$ to $2.5 \mathrm{~nm}$ for a $\mathrm{Gd}_{0.2} \mathrm{Ce}_{0.8} \mathrm{O}_{2-\sigma}$ electrolyte.

As the only carrier for conducting oxygen-ions, oxygen vacancies play a key role in the GB structure. Hojo et al. conducted EELS measurement to confirm the presence of oxygen vacancies at the GB [17]. Lee et al. [18] used the energy dispersive spectroscopy in scanning transmission electron microscopy (STEM-EDS) to acquire a set of STEM-EDS spectra in an area of $20 \times 20 \mathrm{~nm}^{2}$ near a grain boundary. The conversion from the spectra to atomic ratio as a function of position, indicated intensive segregation of both $\mathrm{Gd}^{3+}$ ions and oxygen vacancies segregated toward the GB within 2-3 nm. The composition at the bulk and the grain boundary were thus calculated to be $\mathrm{Gd}_{0.30 \pm 0.02} \mathrm{Ce}_{0.70 \pm 0.02} \mathrm{O}_{1.82 \pm 0.11}$ and $\mathrm{Gd}_{0.36 \pm 0.06} \mathrm{Ce}_{0.64 \pm 0.05} \mathrm{O}_{1.41 \pm 0.17}$ respectively. Besides the segregation of these two defect species, the EELS spectrum from the GB region in [17] is slightly broader than that from the 
grain interior (GI) region, which suggests the presence of $\mathrm{Ce}^{3+}$. This agrees with the finding in [15] that the energy-loss spectra of GDC show that Ce edge is much more rounded at the GB indicating a significant change in local Ce bonding in the GB core. From the extended X-ray absorption fine structure (EXAFS) analysis, energy shift of the Ce edge $\Delta E_{0}$ is found to decrease systematically with increasing doping level, also indicate a partially reduced character of the $\mathrm{Ce}^{4+}$ sites [19]. More studies have also found a large percentage of $\mathrm{Ce}^{3+}$ at GBs and interfaces $[17,16,20]$. A more complete experimental study of doped ceria is conducted in [21]. Here, the three-dimensional compositions at individual grain boundaries were quantified using atom probe tomography(APT) for NDC10 and NDC30. Quantified profiles revealed segregation of dopant and decrease of oxygen concentration near GB.

\subsubsection{Electrical conductivity}

The conductivity of a polycrystalline fluorite-structured oxide arises from conductivity through grains and across GBs. GBs are typically orders of magnitude less conducting than the GI at low and intermediate temperatures. Apart from the presence of impurity phases, space-charge effects are widely accepted as the culprit of decreased conductivity of GBs $[22,23,24]$. Impedance spectroscopy is most commonly employed to study the electrical conductivity of solid electrolytes. Zhou et al. [25] analyzed the impedance spectra of GDC as a function of temperature and grain size. Results reveal that samples with fine grain sizes have higher overall GB resistance. Increasing the temperature to $600^{\circ} \mathrm{C}$, however, eliminates this contribution. In [26], the electronic conductivity of GDC as a function of oxygen partial pressure is investigated to estimate the thermodynamic conditions for the application of GDC as solid electrolyte in IT-SOFCs. The experimental data from both impedance spectroscopy and dc-polarization agrees well. PO2 dependence of the electronic conductivity can be described by $\log \sigma_{e} \propto-\frac{1}{4} \log p\left(O_{2}\right)$. The ionic conductivity is almost independent of $p\left(O_{2}\right)$ with typical values around $0.04 \mathrm{~S} / \mathrm{cm}$ at $700{ }^{\circ} \mathrm{C}$. In the past, researchers have found the GDC conductivity to be ionic under non-reducing conditions. The GB conductivity at $200^{\circ} \mathrm{C}$ is 
$5 \times 10^{4}$ times lower than the grain interior conductivity in GDC [15], clearly demonstrating the deleterious effect of grain boundaries on ionic conductivity especially at low temperatures. This also illustrates the need to develop a fundamental understanding of the relationship between charge transport, GB structure and composition. Such an understanding may allow GB tailoring to be accomplished, leading to substantial improvements in ionic conductivity in polycrystalline electrolytes.

\subsection{Space Charge Theory in Ionic Solids}

The space charge theory in ionic solids has been proposed over 60 years ago [27]. This theory models the GB region as a GB core with specific charge being sandwiched between two space charge regions of opposite charge, which is known as a GB core-space-charge layer model. In oxygen ion conducting solid electrolytes, the positively charged GB core is attributed to accumulation of positively charged oxygen vacancies while the depletion of oxygen vacancies in the space charge region accounts for the negatively charged space charge region. Over the years, researchers have applied various forms of this theory to explain the fact that GB region is orders of magnitude less conductive than the bulk region. For doped systems that consider two species: dopant and one charge-compensating defect (eg., oxygen vacancy), the Gouy-Chapman and Mott-Schottky case are two commonly adopted forms of the space charge theory.

As we shall see from the derivations below, these two varieties of the space charge theory provide analytical solutions for certain characteristic variables of the space charge region, such as the electrostatic potential, the space charge width etc. These analytical tools have been used by researchers to study the conductivity behaviors of doped systems of various dopant concentrations, despite the fact that these models are based on the assumption of only dilute systems. Moreover, derivation of these analytical forms involve several assumptions, including the mobility of charge carriers and the charges of defect species $\left(z_{1}=-z_{2}\right)$, which might not hold for all doped systems. 
The space charge models bridge quantities such as the space-charge potential (the degree to which point defects are depleted or accumulated) and the space-charge screening length (the length scale over which defect profile changes occur) with measurable physical quantities, such as the grain boundary resistance. The charge carrier profile and the space charge potential is linked through the standard Poisson's equation.

$$
\nabla^{2} \varphi=-\rho / \varepsilon
$$

where $\rho$ denotes the charge density, which is obtained from the defect concentration using $\Sigma_{i} z_{i} F c_{i}, \varepsilon$ is the dielectric constant and its spatial variations are ignored.

For dilute systems, the Boltzmann distribution is adopted for ion densities near the interface. This gives the probability of a certain state as a function of that state's energy and temperature of the system. The probability of state $\mathrm{i}$ is given in 2.2 .

$$
p_{i}=\frac{e^{-\epsilon_{i} / k T}}{\sum_{j=1}^{M} e^{-\epsilon_{j} / k T}}
$$

where $p_{i}$ is the probability of state $\mathrm{i}, \epsilon_{i}$ the energy of state $\mathrm{i}, k$ the Boltzmann constant, $T$ the temperature of the system and $M$ the number of all states accessible to the system. Applying to defect concentrations at equilibrium gives the form

$$
c_{i}(x)=c_{i \infty} e^{-\Delta E_{i}(x) / k T}
$$

where $c_{i \infty}$ is the bulk defect concentration, and $\Delta E_{i}(x)$ is the energy of defect species i with reference to the bulk energy.

Combining the above equations with the condition of the constant electrochemical potential leads to the classic Poisson-Boltzmann mode. The one-dimensional form gives $[28]:$

$$
\frac{d^{2}\left(\varphi-\varphi_{\infty}\right)}{d x^{2}}=-\frac{F}{\varepsilon} \Sigma_{i} c_{i \infty} z_{i} \exp \left(-z_{i} F \frac{\varphi-\varphi_{\infty}}{R T}\right)
$$


Here $z_{i}$ is the net charge, $c_{i \infty}$ is the bulk concentration, $\varepsilon$ is the dielectric constant, $k$ is the Boltzmann constant and $T$ is the absolute temperature. The concentration profile for the i species between two locations can be determined by solving the spatial variation of the electrical potential. The current model of space-charge theories assumes that point defects behave as dilute, non-interacting defects. Therefore, the electrochemical potentials of the mobile defects in the bulk and in the GB core take the standard Maxwell-Boltzmann form,

$$
\tilde{\mu}_{d e f}=\mu_{d e f}^{o}+R T \ln \mathrm{n}_{d e f}+\mathrm{z}_{d e f} F \varphi
$$

The second assumption is that material parameters, such as standard defect chemical potentials and charge carrier mobilities, exhibit step functions when traversing from bulk, through the GB, to the bulk. In this case, it is the difference in the standard chemical potential of oxygen vacancies that drives the formation of space-charge zones. The two commonly adopted varieties of traditional space-charge theories are the Gouy-Chapman and Mott-Schottky that have been widely successful in relatively dilute systems.

\subsubsection{Gouy-Chapman}

The Gouy-Chapman case assumes all defect species to be mobile, therefore they are free to redistribute in the space charge regions. Applying appropriate boundary conditions and a reference point for the electrostatic potential (commonly set to be 0 in the bulk), yields the spatial variation of the electrostatic potential under Gouy-Chapman conditions [29]:

$$
\varphi(x)=\frac{2 k T}{z_{i} e} \ln \left(\frac{1+\Theta \exp (-x / \lambda)}{1-\Theta \exp (-x / \lambda)}\right)
$$

where $\lambda$ is the Debye length:

$$
\lambda=\sqrt{\frac{\varepsilon_{0} \varepsilon_{r} k T}{2 z_{i}^{2} e_{2} c_{i \infty}}}
$$


and $\Theta$ is the profile parameter:

$$
\Theta=\tanh \left(\frac{z_{i} e \Delta \varphi}{4 k T}\right)
$$

where $\Delta \varphi=\varphi(0)-\varphi(\infty)$ is the space charge potential, $\varphi(0)$ is the potential at the interface and $\varphi(\infty)$ is the reference value in the bulk. Combining equation 2.3 and 2.6 gives the spatial profiles of defects in the space charge region:

$$
\frac{c_{i}(x)}{c_{i \infty}}=\left(\frac{1+\Theta \exp (-x / \lambda)}{1-\Theta \exp (-x / \lambda)}\right)^{2 z_{i}}
$$

Using these analytical derivations, researchers can estimate defect distributions and interface resistivity for dilute systems under temperatures where all defect species are mobile. Shen et al. implemented a Gouy-Chapman model to investigate the distribution of the accumulated oxygen vacancies in the space charge regions of pure $\mathrm{CeO}_{2}$ [30]. Gregori et al. calculated the concentration of the protons in nanocrystalline ceria thin film right below the water/ceria interface using this approximation [31]. Assuming a Gouy-Chapman situation, Fromling et al. obtained estimations from finite element calculations that approximate experimental results of tracer diffusion profiles of single grains for PZTSr/Nb with different annealing temperatures [32]. Guo et al. applied a Gouy-Chapman situation to a $\mathrm{CaF}_{2} / \mathrm{BaF}_{2}$ heterolayer structure under $773 \mathrm{~K}$ and used the results to qualitively explain its mesoscopic ion conduction behavior [33]. The Gouy-Chapman case is valid only when all defect species can redistribute in the space charge region. This situation, however, changes under reduced temperatures.

\subsubsection{Mott-Schottky}

At reduced temperatures, oftentimes, the defect species controlling the Debye length is insufficiently mobile to redistribute in response to the excess grain boundary charge. In this situation, the Gouy-Chapman case reduces to the Mott-Schottky variety and results in significantly reduced screening. The Mott-Schottky case assumes a flat dopant profile, 
meaning the derivative of dopant (defect species i) concentration is 0 throughout the system, while the other defect species (oxygen vacancy for example), is highly mobile and depleted. In this case, the charge density in Poisson's equation is determined only by the dopant content. If we again neglect the depleted charge carrier, Poisson's equation is simplified and becomes [29]

$$
\frac{d^{2} \varphi}{d x^{2}}=-\frac{z_{i} e c_{i \infty}}{\varepsilon_{r} \varepsilon_{0}}
$$

Combining with boundary conditions $\varphi^{\prime}\left(\lambda^{*}\right)=0$ and $\varphi(\infty)=0$, it yields:

$$
\Delta \varphi(x)=-\frac{z_{i} e c_{i \infty}}{\varepsilon_{r} \varepsilon_{0}}\left(x-\lambda^{*}\right)^{2}
$$

where $\lambda^{*}$ is the depletion(space charge) width:

$$
\lambda^{*}=\sqrt{\frac{2 \varepsilon_{0} \varepsilon_{r} \Delta \varphi(0)}{z_{i} e c_{i \infty}}}=\lambda \sqrt{\frac{4 z_{i} e}{k T} \Delta \varphi(0)}
$$

The relationship between $\lambda$ and $\lambda^{*}$ reveals the difference in space charge width in the MottSchottky case and the Gouy-Chapman case. When the dopant cations cannot redistribute, the space charge width is dependent on the space charge potential, and the depletion width is greater in spatial extent due to a reduced charge screening ability. Combining equation 2.11 with 2.3 for carrier (defect species j) enhancement or depletion gives the concentration dependence:

$$
\frac{c_{j}(x)}{c_{j \infty}}=\exp \left[-\frac{z_{j}}{z_{i}}\left(\frac{x-\lambda^{*}}{2 \lambda}\right)^{2}\right]
$$

In the past, researchers have used Mott-Schottky approximation to estimate interfacial properties and compare with experimental observations. Kim and Maier successfully explained the impedance responses of Gd-doped(0.15 mol\%) and nominally pure nanocrystalline $\mathrm{CeO}_{2-\mathrm{x}}$ and quantitatively analyzed the oxygen partial pressure and temperature dependencies of bulk and boundary conductivities [34, 35]. Guo et al. simulated the meso- 
scopic ion conduction behavior of the $B a F_{2}$ layer model according to the Mott-Schottky defect concentration profiles under 593K [33]. Shirpour et al. analyzed the GB regions of 6 at \% Y- and 6 at \% Sc- doped $\mathrm{BaZrO}_{3}$ prepared by as-sintered Spark Plasma Sintering [36]. The observed dopant concentration approximates the Mott-Schottky situation, however, the calculated GB core charge density largely deviates from the value of a similar low-angle GB in Fe-doped $\mathrm{StTiO}_{3}$ bicrystals although still in the range of physically plausible values.

\subsection{Gap between Experimental Observations and Space Charge Theories}

Past experimental studies have confirmed dopant accumulation near GB but mostly lacked a clear presentation of the anion profiles due to characterization challenges. Theoretical studies using Mott-Schottky or Gouy-Chapman models treated concentrated systems as dilute solutions and draw conclusions with indirect conductivity measurements.

Meijer et al. investigated the local GB composition of polycrystalline ceramics including Y- and Sc- doped $\mathrm{BaZrO}_{3}$ with 6 at\% dopant. The dopant profiles suggests that the accumulation occurs in a region larger than the GB core but the exact location cannot be measured because of the broadening of the electron beam in the specimen. The authors did a rather qualitative explanation of the GB conductivity using the Mott-Schottky and Gouy-Chapman models combined with the dopant profiles, both of which models cannot produce a segregation zone of $>5 \mathrm{~nm}$ as reported and the vacancies are treated as depleted in the GB region [37]. Bowman et al. estimated the GB core cation composition of ceria electrolytes using electron energy-loss spectroscopy( (EELS) in a scanning transmission electron microscope (STEM) and concluded the role of Pr segregation as a co-dopant in enhancing the conductivity [38]. Another experimental study conducted by the same group provided dopant profiles near individual grain boundaries of Ca doped ceria with $2 \%, 5 \%$ and $10 \%$ dopant concentrations [2].

Experimental and theoretical studies that discussed oxygen vacancy distributions near the GB are gradually appearing in literature, and mostly confined to atomistic length scale 
to start with. Lei et al. characterized GBs of fluorite-structured ceramic materials by a combination of Z-contrast imaging and electron energy-loss spectroscopy (EELS) and concluded an indication of increased oxygen vacancies in the grain boundary core [39]. Browning et al. used the same technique with atomic resolution for a direct observation of the GB region of perovskite structured SrTiO3 and fluorite structured Yttria Stabilized Zirconia (YSZ) and found an excess of oxygen vacancies [39]. Lee at al. used the energy dispersive spectroscopy in scanning transmission electron microscopy (STEM-EDS) to conduct atomic-scale characterization of the microstructure and chemical composition near the GBs of GDC thin films. The segregation of dopants and oxygen vacancies along the GBs are observed [40].

Atomic scale characterization of GB defect structure in a YSZ bicrystal using aberrationcorrected TEM also showed significant oxygen deficiency due to segregation of oxygen vacancies near the GB core [41]. Lee at al. developed a hybrid Monte Carlo-molecular dynamics algorithm to simulation the segregation near the surface of YSZ and GDC, resulted in a prediction of dopant segregation near the surface and oxygen vacancy segregation in the first layer beneath the surface and depletion in the subsequent layers [42]. The simulation range is within $3 \mathrm{~nm}$ near the surface. While the prediction for dopants are in accord with experimental results, the profiles of vacancies lacked experimental verification.

In 2016, Diercks et al. performed a three-dimensional quantification of elemental composition for NDC of $10 \%$ and $30 \%$ dopant concentration using atom probe tomography (APT) [43]. This study clearly revealed, for the very first time, the co-accumulation of dopant and oxygen vacancies within an extended region near the GBe.The on-going progress in characterization capabilities has begun to reveal clearer details of defect profiles from near the GB to the bulk, this confirms the fact that dilute theories of Mott-Schottky and GouyChapman cannot treat concentrated systems. Given the limitation of simulation length scale of atomistic simulations, there is a growing demand for a thermodynamic theory that is applicable in concentrated systems. 


\subsection{Variational Phase Field Models}

The phase field method has become a powerful tool to solve interfacial problems at the mesoscale level, where the phase field variable, such as order parameters, can take two distinct values in each of the phases, with a continuous change between values around the interface. Therefore, instead of assuming a sharp interface-properties being discontinuous at the interface, the phase field method accounts for properties evolving continuously around the interface, which is also termed as the diffuse-interface model historically. In the phase field method, the microstructural evolution is modeled using a set of phase-field variables that are continuous functions of time and spatial coordinates [44]. The phase field variables can be conserved or non-conserved. Typical examples of conserved variables are composition variables like molar fractions or concentrations. Such representations have been widely used for spinodal decomposition [45, 46], phase separation of the decomposed structure $[47,48,49,50]$ and precipitation and growth of precipitates [51, 52]. Order parameters and phase-fields are examples of non-conserved variables that are used to distinguish coexisting phases with different structures. A set of order parameters combined with a molar fraction variable, has been frequently adopted to simulate the evolution of ordered precipitates in Ni-based superalloys $[53,54,55,56]$. Generalizing to multi-domain structures, the approach has been used extensively for the study of grain growth $[57,58,59]$ and the coarsening of two-phase structures [60, 61]. After the introduction of the phase-field concept by Langer [62], the single-phase-field representation combined with a temperature and/or composition field was oftentimes employed to study free dentritic growth $[63,64,65]$ in an undercooled melt, cellullar pattern formation during solidification [66, 67] and eutectic growth [68, 69]. Extending the single-phase-field formalism to multiphase systems by Steinbach et al.[70], solidification reactions involving multiple phases, such as eutectic and peritectic solidification $[71,72,73]$ can be modeled. In the phase field method, the equations for the evolution of the phase-field variables are formulated based on general thermodynamic and kinetic principles, therefore, they are distinctly different from first-principle and atomistic studies that deal 
with the behavior of the individual atoms explicitly. This explains the phenomenological characteristic of the phase-field method. Material specific properties are introduced into the model through phenomenological parameters that can be determined based on experimental or theoretical knowledge.

The variational formulations of phase-field models constructs an explicit expression for the free energy of a system. Approximately 60 years ago, Cahn and Hilliard derived a thermodynamic formulation for the free energy of a non-uniform system having a spatial variation in one of its intensive scalar properties, such as composition or density. The free energy is expressed as the sum of two contributions which are functions of the local composition and the composition gradients [74]. Application of variational phase field methods have been employed to model electrochemical systems. In 2003, Bishop et al. conducted a stability analysis of phase separation by spinodal decomposition in ionic solid systems, adopting a Cahn-Hilliard representation for the system free energy [75]. In the following year, a similar thermodynamic approach was proposed by García et al. to derive equilibrium equations and kinetic driving forces for electrically and magnetically active materials [76]. Han et al. descibed Li diffusion in secondary battery electrode, where Li ions reside in interstitial sites of a two-phase topotactic intercalation compound, using a Cahn-Hilliard formulation [77]. Guyer et al. treated an electrochemical interface considering a set of components including cations, anions and electrons and the phase field model successfully captured the charge separation associated with the equilibrium double layer at the interface [78]. The authors further expanded the same phase field model to consider electromigration of electrons and diffusion of cations with time variation, in order to apply for electrodeposition and electrodissolution conditions [79]. This work is an initial demonstration of exploring relationship between double layer structure and interfacial kinetics using variational phase field methods. In [80], Liang et al. introduced a nonlinear phase-field model for modeling electrochemical reactions during nonequilibrium processes at electrode-electrolyte interfaces where the rate of temporal phase-field evolution and the interface motion is considered nonlinear with respect to 
the thermodynamic driving forces. This class of methods also find applications in modeling intercalation processes. In [81], a mathematical model is proposed for ion intercalation in a single crystal of rechargeable-battery composite electrode material $\mathrm{LiFePO}_{4}$. This model incorporated ionic mobility and surface reactions governing ion flux into an existing phasefield formulation and has been utilized to show the spinodal and miscibility gap shrinkage for intercalation in nanoparticles. Combing the Cahn-Hilliard equation with a boundary condition for insertion/extraction kinetics, the general mechanism for the suppression of phase separation in nanoparticles was predicted, including concentration-gradient contributions $[82]$.

\subsection{Nakayama-Martin Oxygen ion conductivity model}

An analytical model for the ionic conductivity of a strongly acceptor doped, fluoritetype oxygen ion conductor was introduced by Martin in 2006 [83]. This model considers the following aspects : 1)the distributions of different cation tetrahedra sites and of oxygen vacancies; 2)the effect of dopant-vacancy interactions on the mobility; 3) the effects

of dopants on the activation barriers for ion migration. The interactions are restricted to only nearest-neighbour and next-nearest-neighbour, and vacancy-vacancy interactions are not considered.

Consider a concentrated solution of host cations A, and dopant cations B . For a statistical cation distribution, the fractions $f_{n}\left(x_{B}\right)$ of tetrahedra containing n B-cations $(\mathrm{n}=0,1,2,3,4)$ can be calculated from combinatorics :

$$
f_{n}\left(x_{B}\right)=2\left(\begin{array}{l}
4 \\
n
\end{array}\right) x_{B}^{n}\left(1-x_{B}\right)^{4-n}
$$

[84] where $x_{B}$ is the site fraction of dopant cations B. Oxygen ions and vacancies can occupy the $2 \mathrm{~N}$ tetrahedra formed by the $\mathrm{N}$ cations. Assuming only nearest neighbor interactions between $V_{O}$ and $\mathrm{B}$, the oxygen vacancies can exist with five different tetrahedral cation 
configurations, which are AAAA, AAAB, AABB, ABBB and BBBB. Using quasi-chemical reactions, the corresponding fractions of those different oxygen vacancies can be expressed as a function of the fraction $V_{A A A A}$ :

$$
\begin{aligned}
{\left[V_{A A A B}\right] } & =\frac{K_{1} \cdot[A A A B] \cdot\left[V_{A A A A}\right]}{[A A A A]+\left(K_{1}-1\right) \cdot\left[V_{A A A A}\right]} \\
{\left[V_{A A B B}\right] } & =\frac{K_{2} \cdot[A A B B] \cdot\left[V_{A A A A}\right]}{[A A A A]+\left(K_{2}-1\right) \cdot\left[V_{A A A A}\right]} \\
{\left[V_{A B B B}\right] } & =\frac{K_{3} \cdot[A B B B] \cdot\left[V_{A A A A}\right]}{[A A A A]+\left(K_{3}-1\right) \cdot\left[V_{A A A A}\right]} \\
{\left[V_{B B B B}\right] } & =\frac{K_{4} \cdot[B B B B] \cdot\left[V_{A A A A}\right]}{[A A A A]+\left(K_{4}-1\right) \cdot\left[V_{A A A A}\right]}
\end{aligned}
$$

where $K_{1}, K_{2}, K_{3}, K_{4}$ are the mass action constants of the four quasi-chemical reactions below:

$$
\begin{aligned}
V_{A A A A}+O_{A A A B} & \longleftrightarrow O_{A A A A}+V_{A A A B}, K_{1}=\frac{\left[O_{A A A A}\right] \cdot\left[V_{A A A B}\right]}{\left[V_{A A A A}\right] \cdot\left[O_{A A A B}\right]}=\exp \left(-\frac{\Delta E_{1}}{k T}\right) \\
V_{A A A A}+O_{A A B B} & \longleftrightarrow O_{A A A A}+V_{A A B B}, K_{2}=\frac{\left[O_{A A A A}\right] \cdot\left[V_{A A B B}\right]}{\left[V_{A A A A}\right] \cdot\left[O_{A A B B}\right]}=\exp \left(-\frac{\Delta E_{2}}{k T}\right) \\
V_{A A A A}+O_{A B B B} & \longleftrightarrow O_{A A A A}+V_{A A A B}, K_{3}=\frac{\left[O_{A A A A}\right] \cdot\left[V_{A B B B}\right]}{\left[V_{A A A A}\right] \cdot\left[O_{A B B B}\right]}=\exp \left(-\frac{\Delta E_{3}}{k T}\right) \\
V_{A A A A}+O_{B B B B} & \longleftrightarrow O_{A A A A}+V_{B B B B}, K_{4}=\frac{\left[O_{A A A A}\right] \cdot\left[V_{B B B B}\right]}{\left[V_{A A A A}\right] \cdot\left[O_{B B B B}\right]}=\exp \left(-\frac{\Delta E_{4}}{k T}\right)
\end{aligned}
$$

Here $\Delta E_{n}(\mathrm{n}=1,2,3,4)$ is the "binding energy" of a vacancy inside a tetrahedron consisting of $\mathrm{n}$ B-cations. This is given by the energy difference between a vacancy in that tetrahedron and a vacancy in an AAAA-tetrahedron.

In order to explain the experimentally observed maximum conductivity and the dependence of the conductivity on the dopant, this model considers the microscopic jump processes of vacancies and their jump rates in detail. Since the B-V interactions have been restricted to nearest-neighbor and next-nearest-neighbor interactions, the vacancy jump rate depends only on the NN/NNN cation configuration of the vacancy before jump and the na- 
ture of the edge to be crossed (A-A, A-B or B-B). The jump frequencies are denoted by $w_{n, m}$ where $\mathrm{n}$ and $\mathrm{m}$ are the numbers of B-cations in the tetrahedron before the jump and in the edge to be crossed.

An oxygen vacancy inside an AAAB-tetrahedron can perform 3 jumps through the three edges with a jump frequency $w_{1,0}$ and 3 jumps through 3 edges with a jump frequency of $w_{1,1}$. The destination tetrahedron must be of the type AAAA,AAAB,AABB or ABBB. So the

probability for the destination tetrahedrons to be accessible are $\left[O_{A A A A}\right]+\left[O_{A A A B}\right]+\left[O_{A A B B}\right]$ and $\left[O_{A A A B}\right]+\left[O_{A A B B}\right]+\left[O_{A B B B}\right]$ respectively, and the partial ionic conductivity owing to vacancies of type $V_{A A A B}$ is given by:

$\left.\sigma_{V_{A A A B}}=\left[V_{A A A B}\right] \cdot\left\{3 \cdot w_{1,0} \cdot\left(O_{A A A A}\right]+\left[O_{A A A B}\right]+\left[O_{A A B B}\right]\right)+3 \cdot w_{1,0} \cdot\left(\left[O_{A A A B}\right]+\left[O_{A A B B}\right]+\left[O_{A B B B}\right]\right)\right\}$

The contributions of the other vacancies are counted for in a similar way.

\subsection{Bayesian Framework for Model Parameterization}

Firstly introduced by Kennedy and O'Hagan, the Bayesian approach was then widely used in calibration and validation of computational models. The general idea of the Bayesian framework can be represented in Equation 2.25. Here $z$ represents experimental observations, $y(x, \theta)$ is the model output as a function of model parameters $\theta$ and the model input $x, \delta(x)$ represent model discrepancy and $\epsilon$ is the observation error.

$$
z=y(x, \theta)+\delta(x)+\epsilon
$$

Model discrepancy comes from the abstraction and simplification of model structures from the real-world situation, the assumptions involved and the insufficient representation of physical or chemical effects etc. $\delta(x)$ is a nonparametric Gaussian process, which can be estimated 
with the BSS-ANOVA framework.

\subsubsection{Parameter Calibration}

Bayesian calibration is a method of drawing conclusions about model parameter distribution or unobserved data through probability statements [85]. Given model parameters and data $\mathrm{y}$, a joint probability distribution for $\theta$ and $\mathrm{y}$ is defined as the multiplication of the prior distribution $\mathrm{p}(\theta)$ and the sampling distribution or data distribution $p(y \mid \theta)$ :

$$
p(\theta, y)=p(\theta) p(y \mid \theta)
$$

According to the basic property of conditional probability known as Bayes' rule, the posterior density is:

$$
p(\theta \mid y)=\frac{p(\theta, y)}{p(y)}=\frac{p(\theta) p(y \mid \theta)}{p(y)}
$$

where $\mathrm{p}(\mathrm{y})=\sum_{\theta} p(\theta) p(y \mid \theta)$. Since $\mathrm{p}(\mathrm{y})$ does not depend on $\theta$ given the data $\mathrm{y}$, it can be treated as a constant. This treatment yields:

$$
p(\theta \mid y) \propto p(\theta) p(y \mid \theta)
$$

where $p(y \mid \theta)$ is called the likelihood function as a function of $\theta$ for fixed data y. The likelihood function can be constructed by computing the product of the probabilities for each data point:

$$
\mathcal{L}(\theta)=\prod_{i=1}^{N} p\left(x_{i} \mid \theta\right)=\prod_{i=1}^{N} p\left(x_{i} \mid \theta\right)
$$

Oftentimes, it is more convenient to compute this on log scale.

$$
\log \mathcal{L}(\theta)=\sum_{i=1}^{N} \log p\left(x_{i} \mid \theta\right)
$$


$\theta$ can be determined by solving the equation $d \log \mathcal{L} / d \theta=0$ so that the likelihood is maximized. The estimation of $\theta$ is based on the observation data. During the calibration process, a sampling strategy is adopted to explore the prior distribution of the parameter space. Markov chain simulation, also known as Markov chain Monte Carlo, or MCMC, is a general method based on drawing values of $\theta$ from appropriate distributions and then correcting those draws to better approximate the target posterior distribution $p(\theta \mid y)$ [86]. The samples are sequentially drawn, with the distribution of the sampled draws depending on the last value drawn. A particular Markov chain algorithm that has been effective for multidimensional problems is the Gibbs sampler, where subvectors of parameter $\theta$ are drawn during each iteration. After a new parameter set is drawn, the corresponding likelihood $\mathcal{L}_{1}$ is calculated and compared with likelihood function $\mathcal{L}_{0}$. If $\mathcal{L}_{1}>\mathcal{L}_{0}$, the new value drawn will be accepted. This process is iterated until the criteria for posterior distribution convergence is satisfied. At this point, the posterior distribution of all parameters are obtained.

Statistically, the Batch Means test is used to judge whether the calibration has reached convergence or not by evaluating the difference between the sample distribution and the target distribution. In the test, after cutting out the samples before burining-in, the total number of left-over samples $\mathrm{N}$, is divided by the number of bins, a, so that each bin has an euqal number of data points $b=N / a$. Then, the mean value for each bin was calculated as below:

$$
\bar{Y}_{j}:=\frac{1}{b} \sum_{l=(j-1) b}^{j b-1} X_{i} \quad \text { for } j=1, \ldots, a
$$

With the number of samples being large enough, the variance of the target distribution can be estimated with the variance of the batch means in Equation 2.32.

$$
\hat{\sigma}_{B M}^{2}:=\frac{b}{a-1} \sum_{j=1}^{a}\left(\bar{Y}_{j}-\bar{X}_{N}\right)^{2}
$$

The half-width confidence interval is given in Equation 2.33. A student's T-test on batch means with a $95 \%$ confidence interval is used to test the convergence: the preferred interval 
is $5 \%$ or less of the sample mean for each estimated parameter.

$$
t_{a_{N}-1} \frac{\hat{\sigma}_{B M}}{\sqrt{N}}
$$

where $t_{a_{N}-1}$ is an appropriate quantile from Student's t distribution with $a_{N}-1$ degrees of freedom.

\subsubsection{Gaussian Processes}

A Gaussian process is a distribution over functions where the mean function is a vector and the covariance function is a matrix. It is a stochastic process that governs the properties of functions. In a GP, for every input $x$, there is an associated function value $f(x)$ which is a random variable. Defining $\mathrm{f}$ to be a GP with mean function $\mathrm{m}$ and covariance function c, we can represent it as:

$$
f \sim G P(m, c)
$$

The GP can define a prior of function $f$ given its function values at finite set of points $x_{1}, x_{2}, \ldots, x_{n}$ being $f\left(x_{1}\right), f\left(x_{2}\right), \ldots, f\left(x_{n}\right)$. The joint distribution of this set of $f\left(x_{1}\right), f\left(x_{2}\right), \ldots, f\left(x_{n}\right)$ is multivariate normal distribution. The covariance matrix $\mathrm{c}$ is given by:

$$
c_{i j}=k\left(x_{i}, x_{j}\right)
$$

where $\mathrm{k}$ is a positive definite kernel function that defines the correlation between two function valus $f\left(x_{1}\right), f\left(x_{2}\right)$ given the input $x_{1}, x_{2}$. Specifically, $c_{i j}$ can be written as:

$$
c_{i j}=\sigma^{2} \exp \left[-\frac{\left(x_{i}-x_{j}\right)^{2}}{\phi^{2}}\right]
$$

Where $\phi$ controls the extent of the correlation between two points, and $\sigma^{2}$ controls the scale of the variance of the function from its mean [85]. The Gaussian process is thus a 
convenient way to represent unknown functions. In a Bayesian framework, the discrepancy functions, being random functions in nature, can be realized through Gaussian processes. Using Gaussian processes to incorporate assumed distributions for discrepancy functions, the posterior distributions of these functions at known input values can be obtained when provided with data for Bayesian calibration [87]. The inversion of the covariance matrix requires the computational complexity of $\mathcal{O}\left(N^{3}\right)$, where $\mathrm{N}$ is the number of data points as the input to the GP. This would exert a challenge for applying Gaussian processes in a complex model.

\subsubsection{The BSS-ANOVA Framework}

Smoothing refers to the estimation of nonparametric functions with stochastic data. The smoothing spline ANOVA models are a class of smoothing methods derived through roughness penalties. This class of methods decomposes the regression function into interpretable main effect and interaction functions. Incorporating the Bayesian method, MCMC sampling can be used to search for models that fit the data well. In this study, the Bayesian Smoothing Spline ANOVA framework is therefore proposed to formulate the discrepancy functions having the properties of traditional Gaussian processes. The covariance function is built from the function components of a functional ANOVA decomposition [88, 89]. By providing a parametric form to estimate nonparametric functions, this approach helps to reduce the stochastic differential equations (SDE) into ordinary differential equations (ODE), which are easier for calibration and uncertainty quantification. It also solves the computa-

tional efficiency issue associated with traditional GPs by scaling linearly with the number of data points [90, 91, 92].

Let's denote the input to the BSS-ANOVA model as $\zeta$ with dimension R. The dis- 
crepancy function can be represented as:

$$
\delta(\zeta)=\beta_{0}+\sum_{r=1}^{R} \delta_{r}\left(\zeta_{r}\right)+\sum_{r<r^{\prime}}^{R} \delta_{r, r^{\prime}}\left(\zeta_{r}, \zeta_{r^{\prime}}\right)+\cdots
$$

It is assumed that $\beta_{0} \sim N\left(0, \varsigma_{0}^{2}\right)$. The main effect functional component is $\delta_{r} \sim$ $G P\left(0, \varsigma_{r}^{2} K_{1}\right)(r=0, \cdots \quad \cdot \quad R)$, with the variance parameter $\varsigma_{r}^{2}$ and the BSS-ANOVA covariance function $K_{1}$ described as below [92]:

$$
K_{1}\left(u, u^{\prime}\right)=B_{1}(u) B_{1}\left(u^{\prime}\right)+B_{2}(u) B_{2}\left(u^{\prime}\right)-\frac{1}{24} B_{4}\left(\left|u-u^{\prime}\right|\right)
$$

where $B_{l}$ is the $l^{\text {th }}$ Bernoulli polynomial. The inputs must be scaled to $[0,1]$ since the covariance function operates in the region of $[0,1]$. Two way interaction functions are assumed to be $\delta_{r, r^{\prime}} \sim G P\left(0, \varsigma_{r}^{2} K_{2}\right)$, where the covariance function $K_{2}$ is the product of the first order kernels, as represented in equation 2.39.

$$
K_{2}\left((u, v),\left(u^{\prime}, v^{\prime}\right)\right)=K_{1}\left(\left(u, u^{\prime}\right),\left(v, v^{\prime}\right)\right)
$$

Similarly, three-way or higher order interaction functional components can be defined. According to Storlie et al.[1], each functional component in 2.37 can be decomposed using Karhunen-Loéve (KL) into an orthogonal basis expansion. Therefore, $\delta_{r}\left(\zeta_{r}\right)$ can be written as below:

$$
\delta_{r}\left(\zeta_{r}\right)=\sum_{i=1}^{\infty} \beta_{r, i} \phi_{i}\left(\zeta_{r}\right), \quad \beta_{r, i} \stackrel{i i d}{\sim} \mathcal{N}\left(0, \tau_{r}^{2}\right)
$$

$\tau_{r}$ is the typical prior standard deviation of $\delta_{r}$ corresponding to a particular variance $\varsigma_{r}^{2}$. The $\phi_{i}$ terms are the eigenfunctions in the KL expansion. As the order of eigenfunction increases, the frequency of the eigenfunction becomes higher and the magnitude decreases. Therefore, it is expected that the terms after some value L are less important and thus can be truncated. The first nine eigenfunctions of the KL expansion for a main effect function 
of the BSS-ANOVA framework are shown in Figure 2.1.
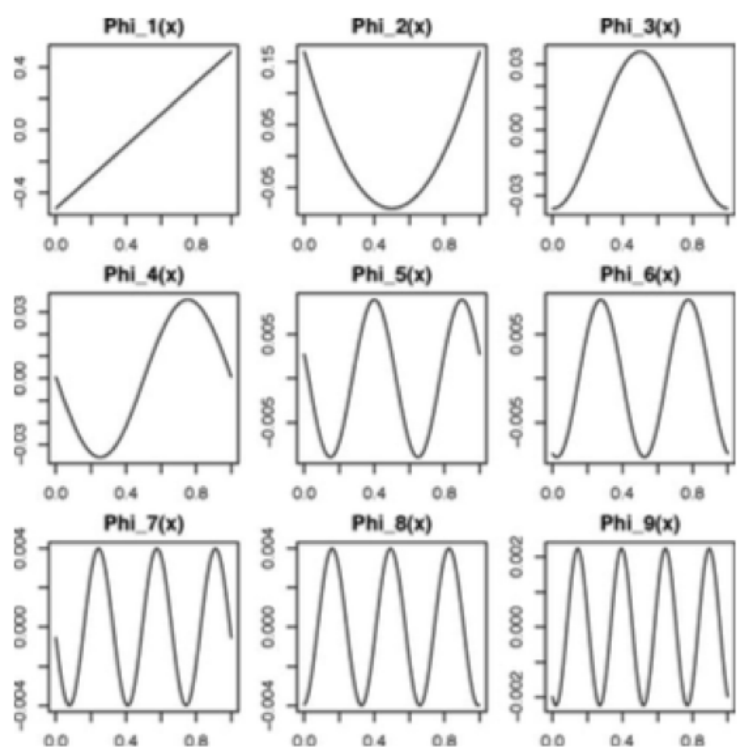

Figure 2.1: Example set of basis functions from [1]

Similarly, the decomposition in Equation 2.40 can be used for two-way and high interactions. The expansion terms for higher-order interactions are the products of the corresponding main effect eigenfunctions. As suggested by Storlie et al., it is sufficient to include only main effects and two-way interactions for many problems. Higher-order interactions can be included if there is lack of fit. Therefore, the overall model in 2.37 can be represented as:

$$
\begin{array}{r}
\delta(\zeta)=\sum_{j=1}^{J} \sum_{i=1}^{I_{\delta i}} \beta_{j, i} \phi_{j, i}(\zeta) \\
\beta_{j, i} \stackrel{i i d}{\sim} \mathcal{N}\left(0, \tau_{j}^{2}\right)
\end{array}
$$

where $j$ indexes over the $J$ functional components included in the discrepancy function, and $i$ indexes over the number of basis function $I_{\delta i}$ used for the $j^{\text {th }}$ functional component of the discrepancy representation. The $\beta_{j, i}, \phi_{j, i}$, and $\tau_{j}$ have corresponding meanings as in the expansion of (2.40) for the $j^{\text {th }}$ functional component. 


\section{CHAPTER 3}

\section{A GENERALIZED SPACE CHARGE THEORY: POISSON-CAHN}

Conventional space charge calculations such as Gouy-Chapman or Mott-Schottky variety, although being successfully applied in relatively dilute systems, have shown inadequacy when considering highly-doped systems. For example, co-accumulation of dopant cations and oxygen vacancies which has been experimentally observed, cannot be modeled with the traditional space charge theory. Segregation layers on the orders of $10 \mathrm{~nm}$ in a concentrated solution also cannot be explained. Despite these theoretical limitations, highly-doped systems are more related to industrial applications. Therefore, a new thermodynamic framework that is applicable to systems with high solute concentrations must take its place. Past research findings have shown the non-negligible influences of defect interactions and gradient energy in highly concentrated systems, this framework therefore should incorporate effects of these factors.

\subsection{Defect Interactions}

Moving from dilute systems to concentrated solid solutions, defect interactions including self-interactions and across-species interactions can no longer be ignored. The importance of defect interactions can be inferred from the behavior of ionic conductivity in these doped systems. It is widely known that the ionic conductivity $\sigma$ of $\mathrm{CeO}_{2}-\mathrm{M}_{2} \mathrm{O}_{3}$, does not increase monotonically with increasing the concentration of charge carrier, i.e. oxygen vacancy. For example, Yahiro et al. found a maximum in ionic conductivity of samarium doped ceria (SDC) at approximately 20atom\% [93]. A lot of experimental and theoretical research was conducted in recent years to reveal the exact reason. One explanation for the conductivity decrease after the early peak, is the attraction of oxygen vacancies to the dopant ions which 
leads to the trapping of oxygen vacancies and thus lowered oxygen ion mobility. Furthermore, the repulsion and ordering of the oxygen vacancies can contribute to the reasoning $[94,95]$. Another possible explanation is the increased energy barrier of migration for oxygen vacancies adjacent to neighboring $\mathrm{M}^{3+}[96,97,98,99]$. For example, rare earth ions with larger ionic radii can result in a higher jump barrier for oxygen ions. The occupation of the "migration edge" formed by these neighboring cations increases the edge energy for the respective jump.

Therefore, the Poisson-Cahn theory incorporates defect interactions to the overall free energy of the system, in the form of excess free energy. From the standpoint of electrochemical potential, these local terms together add an excess chemical potential term to the electrochemical potential of each species.

Taking rare-earth doped ceria as a model system, defect interactions to be incorporated are dopant self-interaction $\left(f_{y y}\right)$, vacancy self-interaction $\left(f_{v v}\right)$ and dopant-vacancy interaction $\left(f_{y v}\right)$. Considering the existence of polarons in doped ceria, electron self-interaction $\left(f_{q q}\right)$, dopant-electron interaction $\left(f_{y q}\right)$ and vacancy-electron interaction $\left(f_{v q}\right)$ also need to be included in the free energy formalism.

Experimental measurements of defect association energies have been done for a few rare earth oxides. Gerhardt-Anderson and Nowick [94] determined the association energies of several RE-V pairs from conductivity data: $-0.67 \mathrm{eV}$ for $\mathrm{Sc}-\mathrm{V}$ pair, $-0.21 \mathrm{eV}$ for $\mathrm{Y}-\mathrm{V}$ pair, $-0.12 \mathrm{eV}$ for $\mathrm{Gd}-\mathrm{V}$ pair and $-0.14 \mathrm{eV}$ for La-V pair. In another study conducted by Wang et al. [100], the associate energy of the Y-V pair was calculated using impedance spectroscopy to be $-0.43 \mathrm{eV}$. Computational calculations based on DFT were obtained by Grieshammer et al [101]. : $-0.75 \mathrm{eV}$ for $\mathrm{Sc}-\mathrm{V}$ pair, $-0.35 \mathrm{eV}$ for $\mathrm{Y}-\mathrm{V}$ pair, $-0.29 \mathrm{eV}$ for Gd-V pair and -0.12 eV for La-V pair. These energies were calculated for various supercells considering the nearest $(1 \mathrm{NN})$ and next nearest neighbour $(2 \mathrm{NN})$, and extrapolated to infinite dilution.Therefore, discrepancies between experimental and computation results are expected due to the concentration difference involved in each case. These interaction energies of RE- 
$\mathrm{V}$ pair are strictly negative indicating an attractive interaction between the rare earth ions and the vacancies as expected from the corresponding electrostatics due to the opposite relative charges of rare earth ions and oxygen vacancies. Andersson et al. have also found the influence on the association energy by elastic effects as the attraction of the RE-V pair decreases with increasing radius. Grieshammer et al. [101] also calculated the Coulomb energy for the RE-V interaction in $1 \mathrm{NN}$ and $2 \mathrm{NN}$ positions for various systems. The results showed large energy differences in $1 \mathrm{NN}$ positions and about the energy level at about -0.2 $\mathrm{eV}$ (close to the Coulomb energy of $-0.25 \mathrm{eV}$ ) for all rare earth ions, suggesting that the elastic effect is more profound only for the $1 \mathrm{NN}$ position and rapidly decays with distance.

The nearest neighbor association of oxygen vacancies was calculated to be $0.77 \mathrm{eV}$ by Nakayama and Martin [96], and $0.90 \mathrm{eV}$ by Grieshammer et al. [101] The difference can be explained by the finite-size correction adopted in the DFT simulations. The association energies of two oxygen vacancies in ceria depending on the distance displayed a decreasing trend from the $1 \mathrm{NN}$ to $5 \mathrm{NN}$ in the range of $1.2 \mathrm{eV}$ to $0.3 \mathrm{eV}$ [101]. This is in agreement with the general trend found by Ismail et al in investigating the defect interactions in samarium doped ceria using DFT $+\mathrm{U}$ methods [102]. However, in the later study, they found a more rapid decrease of the interaction energy after the $1 \mathrm{NN}$, an equal value for $2 \mathrm{NN}$ and $4 \mathrm{NN}$ and an near-zero energy minimum for the $5 \mathrm{NN}$ position. Positive values here imply the repulsive interaction between oxygen vacancies. The association energies of two rare-earth ions (RE$\mathrm{RE}$ ) calculated for the first coordination shell is around $0.10 \mathrm{eV}$ for a few rare-earth oxides [101]. This implies that the interaction is mainly due to Coulomb repulsion between dopant ions and contribution from elastic effects is minimal.

\subsection{Gradient Energy Contribution}

The concept of gradient energy was first introduced by John W. Cahn and John E. Hilliard in 1958 [74] in deriving the free energy of a nonuniform system. The system possesses the property of having a spatial variation in one of its intensive scalar properties, such as 
composition or density. Considering a binary solution system and the nonuniform property being $c$, the mole fraction of the $\mathrm{B}$ component, the local free energy per molecule, $f$, in a region of nonuniform composition will depend both on the local composition and on the composition of the immediate environment. Therefore, $f$ can be expressed as the sum of two contributions, which are functions of the local composition and the local composition derivatives, respectively. Providing $f$ is a continuous function of these variables, it can be expanded in a gradient expansion about $f_{0}$, where the coefficients of the expansion are derivatives of $f$ with respect to increasing orders of derivatives of $c$. Here $f_{0}$ is the free energy per molecule of a solution of uniform composition. Assuming that the local free energy $f$ is a function only of $f_{0}$, the composition and its derivatives, then since $\mathrm{f}$ must be a scalar invariant with respect to the direction of the gradient, only terms in even powers of the operator $\nabla$ can appear. The leading terms of $\mathrm{f}$ must therefore be of the form:

$$
f\left(c, \nabla c, \nabla^{2} c, \ldots\right)=f_{0}(c)+k_{1} \nabla^{2} c+k_{2}(\nabla c)^{2}+\ldots
$$

Integrating over a volume $\mathrm{V}$ of the solution gives the total free energy $\mathrm{F}$ of this volume:

$$
F=N_{v} \int_{V} f d V=N_{v} \int_{V}\left[f_{0}(c)+k_{1} \nabla^{2} c+k_{2}(\nabla c)^{2}+\ldots\right] d V
$$

where $N_{v}$ is the number of molecules per unit volume. Applying the divergence theorem gives:

$$
\int_{V}\left(k_{1} \nabla^{2} c\right) d V=-\int_{V}\left(d k_{1} / d c\right)(\nabla c)^{2} d V+\int_{S}\left(k_{1} \nabla c \cdot n\right) d S
$$

Not concerning with effects at the external surface, a boundary condition of integration in Equation 3.2 can be chosen so that $\nabla c \cdot n$ is zero at the boundary. The surface integral then vanishes and Equation 3.3 can be used to eliminate the term $\nabla^{2} c$ from Equation 3.2 to yield [74]:

$$
F=N_{v} \int_{V} f d V=N_{v} \int_{V}\left[f_{0}(c)+k(\nabla c)^{2}+\ldots\right] d V
$$


where

$$
k=-d k_{1} / d c+k_{2}
$$

Equation 3.4 is the central form of the nonuniform system treatment. It shows that the free energy of a small volume of nonuniform solution can be expressed as the sum of two contributions, one being the free energy that this volume would have in a homogeneous solution and the other a "gradient energy" which is related to the derivatives of the local composition.

The gradient energy contribution comes from breaking of symmetry relative to the spatially homogeneous case. This is a key concept in the Cahn-Hilliard theory for inhomogeneous systems and past research using variational phase field models have manifested the significance of gradient energy contributions. When solute concentrations are no longer spatially uniform, a gradient in the concentration is associated with a strictly positive contribution to the free energy.

To describe the dynamics of surface enrichment in binary mixtures, Binder and Frisch developed a mean-field theory in the framework of a lattice model, where a gradient expansion was used to derive the nonlinear differential equation for the Kawasaki spin-exchange model [103]. In analyzing the surface effect and the interplay between wetting and finite-size effect in binary systems of confined geometries, Binder et al. concluded that the chemical-potential gradient drives one component into the bulk whether phase separation is present or not [104]. Puri and Binder have employed a phenomenological theory for surface effects in mixtures combining the Cahn-Hilliard equation and appropriate boundary conditions considering the one-sided gradient effect [105]. This theory has been applied to binary mixtures including solid binary mixtures to study surface effects on spinodal decomposition and its interplay with wetting phenomena [106]. Binder et al. have also shown that the gradient energy in the Cahn-Hilliard formalism can be related to lattice models such as the Kawasaki spinexchange kinetic Ising model [107]. These works have provided valuable insights on the critical role of gradient effects in non homogeneous systems. The gradient energy coefficient 
that determines the surface enrichment layer is analogous to its effect in controlling the ion segregation layer near the interface of doped systems.

Cahn-Hilliard derived an expression for a composition-independent gradient energy coefficient $(\kappa)$ applicable to a cubic system [74]. This has provided a basis for estimating the gradient energy coefficient from thermodynamic data of homogeneous systems.

Han et al. applied a phase field model to describe Li diffusion in secondary battery electrodes, where a value of $2.48 \times 10^{-11} \mathrm{~J} / \mathrm{m}$ is derived for a one-dimensional lattice of $\mathrm{LiFePO}_{4}$ [108]. The authors did use two other values an order of magnitude smaller and larger than the base value to evaluate the effect on diffusion coefficient, showing that both these values didn't cause significant deviation between measured diffusion constant and the effective chemical diffusion coefficient when the gradient energy is not zero . Lass et al. derived an expression of $\kappa$ for a binary, face-centered cubic crystal considering pair-wise, three-body and four-body nearest neighbor interactions. Their calculated values of $(\mathrm{k})$ are within the range between $1.20 \times 10^{-11}$ and $1.02 \times 10^{-} 10 \mathrm{~J} / \mathrm{m}$ for a Al-Zn system [109]. Their reported values are in agreement with the theoretical calculations of $1.8 \pm 0.3 \times 10^{-11}$ by Rundman and Hilliard [110], while differs from the measured value of $16 \pm 3 \times 10^{-11}$. In [111], a first-principle study based on the cluster variation method is used to compute $\kappa$

for interface boundaries in Ag-Al. The calculated value decreases from $6.1 \times 10^{-11} \mathrm{~J} / \mathrm{m}$ to $4.5 \times 10^{-11}$ for an $\mathrm{Al}$ concentration of 81 at. \% when temperature increases from $500 \mathrm{~K}$ to $900 \mathrm{~K}$. At temperature of $600 \mathrm{~K}$, the value of $\kappa$ increases from $2.5 \times 10^{-11}$ to $21 \times 10^{-11}$ with the increase of $\mathrm{Al}$ concentration from 0.55 to 0.99 . Hoyt did a molecular dynamics study for an embedded atom method model of $\mathrm{Cu}-\mathrm{Pb}$ in $5.3 \mathrm{~nm}$ liquid droplet, and found that $\kappa$ lies in the range of $\left(1.0-1.4 \times 10^{-10} \mathrm{~J} / \mathrm{m}\right)$ for three different temperatures evaluated [112]

\subsection{Derivation of the Poisson-Cahn Model}

Taking inspiration from the Cahn-Hilliard theory for the solute segregation in alloys and the Cahn theory of wetting a solid-liquid interface, a generalized space charge theory is 
proposed as the "Posson-Cahn" theory. The Poisson-Cahn theory replaces the Boltzmann model for the local ion density with the Cahn-Hilliard theory of inhomogeneous systems, and includes defect interactions and gradient energy contribution to the free energy of systems: two factors that are particularly influential in non-dilute systems. This makes it possible for this theory to be applied to doped systems of all levels of concentrations.

The global approach of thermodynamic treatment, employed by many authors, constructs an expression for the free energy of the entire system and then minimizes to yield equations for the local concentrations of the various defects. Taking a grain boundary model system of $\mathrm{CeO}_{2}-\mathrm{M}_{2} \mathrm{O}_{3}$ with dopant ions, oxygen vacancies and electrons, the free energy function of the system can be formulated as below:

$$
\begin{array}{r}
\Omega[y, v, q, \varphi ; T]=\Phi(y(0), v(0), q(0), T)+\int_{0}^{L}\left(W(y, v, q, T)+\frac{1}{2} c_{y}\left(\frac{d y}{d x}\right)^{2}+\frac{1}{2} c_{v}\left(\frac{d v}{d x}\right)^{2}\right. \\
\left.+\frac{1}{2} c_{q}\left(\frac{d q}{d x}\right)^{2}-\frac{1}{2} \varepsilon_{r} \varepsilon_{0}\left(\frac{d \varphi}{d x}\right)^{2}+F \varphi\left(2 n_{o s} v-n_{c s} y-n_{c s} q\right)\right) d x
\end{array}
$$

Here we consider a one-dimensional symmetric grain model where $\mathrm{x}=0$ represents the grain boundary position, and $\mathrm{x}=\mathrm{L}$ represents the position of the half grain domain. For example, if the whole grain has a size of $800 \mathrm{~nm}$, L would be $400 \mathrm{~nm}$ given the symmetric grain approximation. The free energy density $\mathrm{W}$ is a double-well potential in $\mathrm{y}, \mathrm{v}$, and $\mathrm{q}$ arising from excess free energy terms combined with site-limited entropy. Self-interactions and interactions between different types of defects are included:

$$
\begin{array}{r}
W(y, v, q, T)=n_{\mathrm{cs}} f_{\mathrm{yy}} y^{2}+n_{\mathrm{os}} f_{\mathrm{vv}} v^{2}+n_{\mathrm{cs}} f_{\mathrm{qq}} q^{2}+f_{\mathrm{yv}} y v+f_{\mathrm{yq}} y q+f_{\mathrm{vq}} v q \\
+f_{\mathrm{v}}^{0} v+f_{\mathrm{q}}^{0} q+n_{\mathrm{cs}} R T[y \log (y)+(1-y-q) \log (1-y-q)] \\
+n_{\mathrm{os}} R T[v \log (v)+(1-v) \log (1-v)]+n_{\mathrm{cs}} R T[q \log (q)+(1-y-q) \log (1-y-q)]
\end{array}
$$

The interface free energy density includes terms pertaining to the preference for dopant 
cations, oxygen vacancies and electrons to reside at the grain boundary:

$$
\Phi(y(0), v(0), q(0), T)=n_{\mathrm{o}} f_{\mathrm{o}} v(0)+\frac{1}{2} n_{\mathrm{o}} f_{\mathrm{ds}} y(0)+\frac{1}{2} n_{\mathrm{o}} f_{\mathrm{qs}} q(0)
$$

where $n_{\mathrm{o}}$ is the number of oxygen sites per unit area at the interface, $f_{\mathrm{o}}$ is the vacancy segregation energy, $f_{\mathrm{ds}}$ and $f_{\mathrm{qs}}$ are the affinities for dopants and electrons for the interface, respectively. Minimization of the system free energy function $\Omega$ involves taking variational differentiation of the functional with respect to $\mathrm{y}, \mathrm{v}, \mathrm{q}$, and $\varphi$ subjecting to the constraints of mass conservation and electroneutrality condition in the bulk. Thus, the Euler-Lagrange equations can be obtained as below:

$$
\begin{array}{r}
n_{\mathrm{cs}} F \varphi+2 n_{\mathrm{cs}} f_{\mathrm{yy}} y+f_{\mathrm{yv}} v+f_{\mathrm{yq}} q+n_{\mathrm{cs}} R T \log \frac{y}{1-y-q}-2 c_{\mathrm{y}} \frac{d^{2} y}{d x^{2}}=0 \\
n_{\mathrm{os}} F \varphi+2 n_{\mathrm{os}} f_{\mathrm{vv}} v+f_{\mathrm{yv}} y+f_{\mathrm{vq}} q+n_{\mathrm{os}} R T \log \frac{v}{1-v}-2 c_{\mathrm{v}} \frac{d^{2} v}{d x^{2}}=0 \\
n_{\mathrm{cs}} F \varphi+2 n_{\mathrm{cs}} f_{\mathrm{qq}} q+f_{\mathrm{vq}} v+f_{\mathrm{yq}} y+n_{\mathrm{cs}} R T \log \frac{q}{1-y-q}-2 c_{\mathrm{q}} \frac{d^{2} q}{d x^{2}}=0 \\
\frac{d^{2} \varphi}{d x^{2}}=\frac{-F}{\varepsilon_{0} \varepsilon_{\mathrm{r}}}\left(2 n_{\mathrm{os}} v-n_{\mathrm{cs}} y-n_{\mathrm{cs}} q\right)
\end{array}
$$

The boundary conditions arising naturally from the variational analysis are:

$$
\begin{array}{r}
\left.n_{\mathrm{cs}} c_{\mathrm{y}} \frac{d y}{d x}\right|_{x=0}=\frac{1}{2} n_{\mathrm{o}} f_{\mathrm{ds}} \\
\left.n_{\mathrm{os}} c_{\mathrm{v}} \frac{d v}{d x}\right|_{x=0}=n_{\mathrm{o}} f_{\mathrm{o}} \\
\left.n_{\mathrm{cs}} c_{\mathrm{q}} \frac{d q}{d x}\right|_{x=0}=\frac{1}{2} n_{\mathrm{o}} f_{\mathrm{qs}} \\
\left.\frac{d \varphi}{d x}\right|_{x=0}=0
\end{array}
$$

Another equivalent route to this is to directly define suitable electrochemical potentials for the defect species and then require proper behavior of these potentials in thermodynamic equilibrium or kinetic situations [113]. Adopting this approach, the electrochemical 
potentials of the defect species in the "Posson-Cahn" form are thus formulated as:

$$
\begin{gathered}
\tilde{\mu}_{\mathrm{y}}=\mu_{\mathrm{y}}^{o}+f_{\mathrm{y}} y+f_{\mathrm{yv}} v+f_{\mathrm{yq}} q+R T \ln \left(\frac{y}{1-y-q}\right)-F \varphi-c_{y} \frac{d^{2} y}{d x^{2}} \\
\tilde{\mu}_{\mathrm{v}}=\mu_{\mathrm{v}}^{o}+f_{\mathrm{v}} v+f_{\mathrm{yv}} y+f_{\mathrm{vq}} q+R T \ln \left(\frac{v}{1-v}\right)+2 F \varphi-c_{v} \frac{d^{2} v}{d x^{2}} \\
\tilde{\mu}_{\mathrm{q}}=\mu_{\mathrm{q}}^{o}+f_{\mathrm{q}} q+f_{\mathrm{yq}} y+f_{\mathrm{vq}} v+R T \ln \left(\frac{q}{1-y-q}\right)-F \varphi-c_{q} \frac{d^{2} q}{d x^{2}}
\end{gathered}
$$

Here the formulation of the electrochemical potential includes the standard electrochemical potential, terms for interaction energies ( $f$ terms), terms associated with gradient effects ( $c$ terms), configurational and electrostatic terms. The local $(f)$ and non-local $(c)$ terms together comprise an excess chemical potential contributing to the electrochemical potential formulation. In equilibrium, the electrochemical potentials remain constant throughout the system. Thus, setting the corresponding derivatives to zero gives rise to the same set of equations as obtained through the variational analysis. 


\section{CHAPTER 4}

\section{KINETIC MODELING FOR DOPANT SEGREGATION}

\subsection{Kinetic Model Development}

In the aliovalently doped ceria system, dopant cations and oxygen vacancies approach equilibrium state at vastly different speed due to the large difference in diffusivity. It is widely known that anion diffusivity is orders of magnitude faster than cations, therefore dopant profile is expected to go through a kinetic process while oxygen vacancy has already established its equilibrium profile. There is currently no available experimental or theoretical studies that can be used to describe the kinetic process of dopant segregation. Therefore, a kinetic model is needed to reveal the dopant profile development before reaching equilibrium. The kinetic model originates from the flux equation where the flux $J$ is assumed to be proportional to the gradient of the electrochemical potential, $\nabla \mu$, as is usually done in kinetic theory, then the flux of species $i, J_{i}$, can be written as:

$$
J_{i}=-u \bar{c} \nabla \mu
$$

Where $u$ is the mobility of species $i, \bar{c}$ is the average concentration. Given the flux term, the continuity equation expressing the conservation of diffusing species becomes

$$
\frac{d c}{d t}=-\nabla \cdot J_{i}
$$

The expression for electrochemical potential $\mu$ here follows the Poisson-Cahn formation in Equation 3.17. In this model development, we consider the mobility of the dopant cations, 
therefore the governing equation for the non-equilibrium state is

$$
\frac{d y}{d t}=\bar{y} u_{\mathrm{y}}\left(\frac{d^{2} \tilde{\mu}_{\mathrm{y}}}{d x^{2}}-c_{\mathrm{y}} \frac{d^{4} y}{d x^{4}}\right)
$$

where $y$ is the site fraction of dopant cations, $u_{\mathrm{y}}$ is the cation mobility, $\bar{y}$ is the average site fraction of cation sites in the bulk (an approximation adopted for the flux term), $c_{\mathrm{y}}$ is the gradient energy coefficient and $\tilde{\mu}_{\mathrm{y}}$ the electrochemical potential of dopant cations, minus gradient effects. For oxygen vacancies, following the assumption that this species remains constantly in equilibrium, the governing equation becomes

$$
\tilde{\mu_{\mathrm{v}}}-c_{\mathrm{v}} \frac{d^{2} v}{d x^{2}}=0
$$

where $\tilde{\mu}_{\mathrm{v}}$ is the electrochemical potential of oxygen vacancies, minus gradient effects, $c_{\mathrm{v}}$ is the gradient energy coefficient, and $v$ is the vacancy site fraction. In Equation 4.3 and 4.4, electrochemical potentials of defect species are

$$
\begin{gathered}
\tilde{\mu}_{\mathrm{y}}=\mu_{\mathrm{y}}^{o}+f_{\mathrm{y}} y+f_{\mathrm{yv}} v+R T \ln \left(\frac{y}{1-y}\right)-F \varphi \\
\tilde{\mu}_{\mathrm{v}}=\mu_{\mathrm{v}}^{o}+f_{\mathrm{v}} v+f_{\mathrm{yv}} y+R T \ln \left(\frac{v}{1-v}\right)+2 F \varphi
\end{gathered}
$$

where the subscript $\mathrm{y}$ stands for dopant cations and $\mathrm{v}$ stands for oxygen vacancies. A complete description of the parameters appeared in the formulation can be found in Table 4.1. The electrostatic potential is governed by Poisson's equation:

$$
\frac{d^{2} \varphi}{d x^{2}}=-\frac{F}{\varepsilon_{r} \varepsilon_{0}}\left(2 n_{\mathrm{v}} v-n_{\mathrm{y}} y\right)
$$

where $\varepsilon_{r}$ is the relative permittivity, $\varepsilon_{0}$ the permittivity of free space, $n_{\mathrm{v}}$ and $n_{\mathrm{y}}$ are the concentrations of oxygen sites in the bulk, and cation sites in the bulk, respectively. 
In solving this system of equations the following boundary conditions apply:

$$
\begin{gathered}
\left.\frac{d y}{d x}\right|_{x=0}=\left.\frac{d y}{d x}\right|_{x=L}=0 \\
\left.\frac{d w_{y}}{d x}\right|_{x=0}=\left.\frac{d w_{y}}{d x}\right|_{x=L}=0 \\
\left.c_{v} \frac{d v}{d x}\right|_{x=0}=n_{0} f_{0} \\
\left.c_{v} \frac{d v}{d x}\right|_{x=L}=0 \\
\left.\frac{d \varphi}{d x}\right|_{x=0}=0 \\
\varphi(L)=0
\end{gathered}
$$

where $x=0$ pertains to the interface and $x=L$ the grain center, which is $400 \mathrm{~nm}$ in this study. $n_{0}$ is the concentration of oxygen sites at the interface, $w_{y}$ is the test function used for the finite element discretization of the dopant equation, and $f_{0}$ is the segregation energy for vacancies.

The mobility $u_{\mathrm{y}}$ was estimated from experimental data appearing in References $[114$, 115, 116]. These references contain diffusivity data derived from probing cation transport directly [114] or examining cation diffusion indirectly (by studying grain-growth kinetics) [115] in doped ceria. An Arrhenius model for the diffusivity was fitted to the data and the corresponding parameters for the mobility were found using the Nernst-Einstein relation. Since the dopant cation in GDC diffuse through a vacancy mechanism, its mobility varies with local cation concentration. This implementation starts with a simplified treatment of the mobility parameter being a constant, not to divert attention away from the first presentation of this kinetic model.

Combining Equation 4.3 with Equation 4.5, the final governing equation for dopant defect is a $4^{\text {th }}$ order nonlinear equation. Finite element method with cubic basis functions is applied to represent the spatial domain and Crank-Nicolson scheme is used for time discretization. Equation 4.4 and 4.7 are linearized using finite difference method with 2nd 
order accuracy,similar to a previous development for the equilibrium case [13]. Newton's method is used to solve the final equations for defect site fractions and electrochemical potential.Parameter values can be found in Table 4.1.

\begin{tabular}{lll} 
Param. & Value & Indication \\
\hline$n_{0}$ & $1.0 \times 10^{-5} \mathrm{~mol} / \mathrm{m}^{2}$ & interfacial site density for vacancies \\
$f_{\mathrm{v}}$ & $0.9 \mathrm{eV}[117]$ & vacancy self-interaction energy \\
$f_{0}$ & $-2.5 \mathrm{eV}$ & vacancy segregation energy \\
$f_{\mathrm{y}}$ & $0.9 \mathrm{eV}$ & dopant self-interaction \\
$f_{\mathrm{yv}}$ & $-0.07 \mathrm{eV}[12]$ & dopant-vacancy interaction \\
$c_{\mathrm{v}}$ & $0.124 \mathrm{eV}-\mathrm{nm}^{2}$ & vacancy gradient energy coefficient \\
$c_{\mathrm{y}}$ & $2.0 \mathrm{eV}-\mathrm{nm}^{2}$ & dopant gradient energy coefficient \\
$\varepsilon_{r}$ & 35.0 & relative permittivity \\
$u_{\mathrm{y}}$ & $3.15 \times 10^{-22} \mathrm{~mol}-\mathrm{m}^{2} / \mathrm{J} / \mathrm{s} / \mathrm{K}$ & cation mobility
\end{tabular}

Table 4.1: Model parameter values

\subsection{Model Results and Discussion}

The kinetic model developed based on the Poisson-Cahn theory was applied to GDC of different dopant concentrations: $20 \%, 1 \%$ and $0.1 \%$. The results for $1300{ }^{\circ} \mathrm{C}$, in terms of the behavior of the defect concentrations and electrostatic potential as a function of time, are typical of those at other concentrations, with the sole difference being the time scale. The following results thus pertain to GDC with $20 \%$ dopant under at $1300{ }^{\circ} \mathrm{C}$.

At $1300{ }^{\circ} \mathrm{C}$, and starting from an initial state of a uniform defect concentration, the model shows that appreciable dopant segregation starts on a surprisingly short time scale of $10^{-8}$ seconds. Dopant first segregates close to the interface, creating a depletion zone as shown in Figure 4.1. The thickness and depth of the depletion zone both increase with time. 


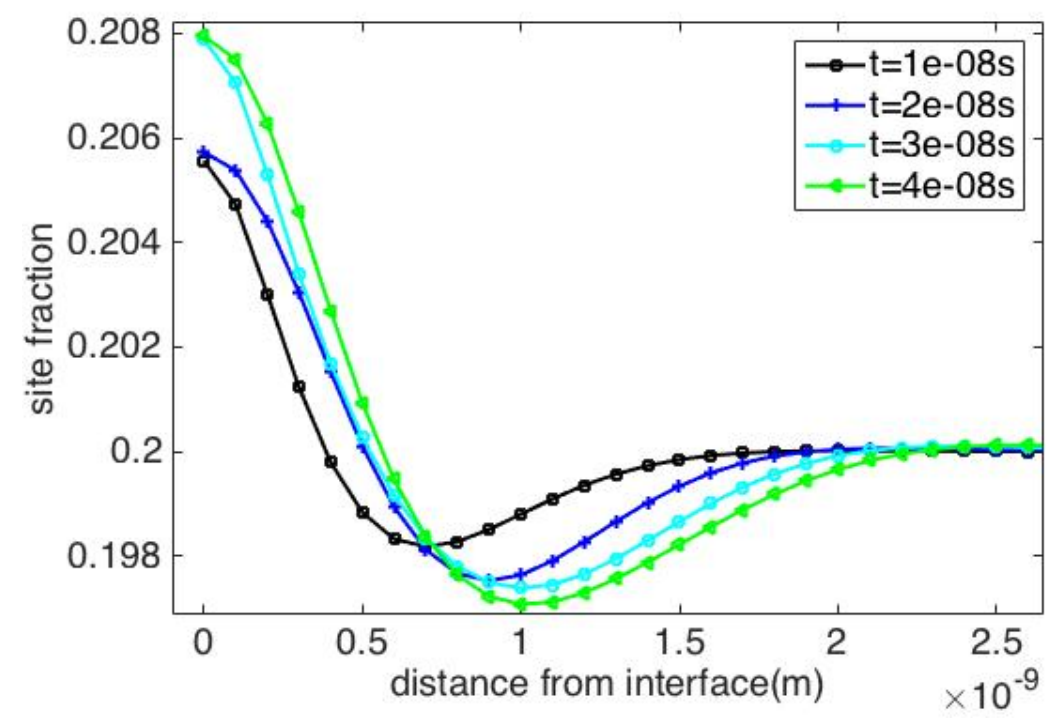

Figure 4.1: Dopant profile as a function of distance from the interface at $1300{ }^{\circ} \mathrm{C}$ and $20 \%$ dopant cation site fraction, during the initial $10^{-7} \mathrm{~s}$ of annealing time

A second segregation zone appears as shown in Figure 4.2, the extent of which initially increases with time. This may be attributed to the fact that the amount of dopant is relatively high than the dilute case and dopant cations that haven't segregated to the GB are likely to temporarily segregate to its vicinity. Shortly after its appearance, however, the second segregation zone gradually starts diminishing, as shown in Figure 4.3, as more dopant cations segregate to the interface from its vicinity. This second accumulation region occurs only in the more concentrated solid solutions, as the second accumulation zone didn't manifest itself for the $1 \%$ and $0.1 \%$ doped GDC. 


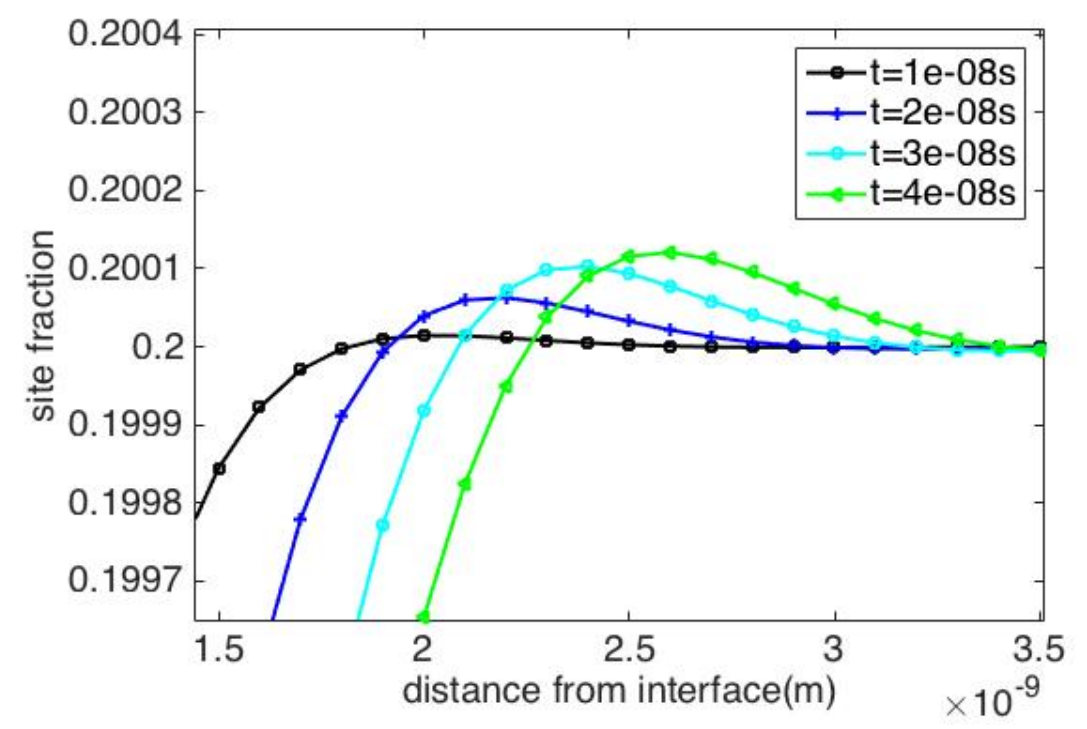

Figure 4.2: Dopant profile as a function of distance from the interface at $1300{ }^{\circ} \mathrm{C}$ and $20 \%$ dopant cation site fraction, during the initial $10^{-7} \mathrm{~s}$ of annealing time (zoomed in to show the second segregation zone)

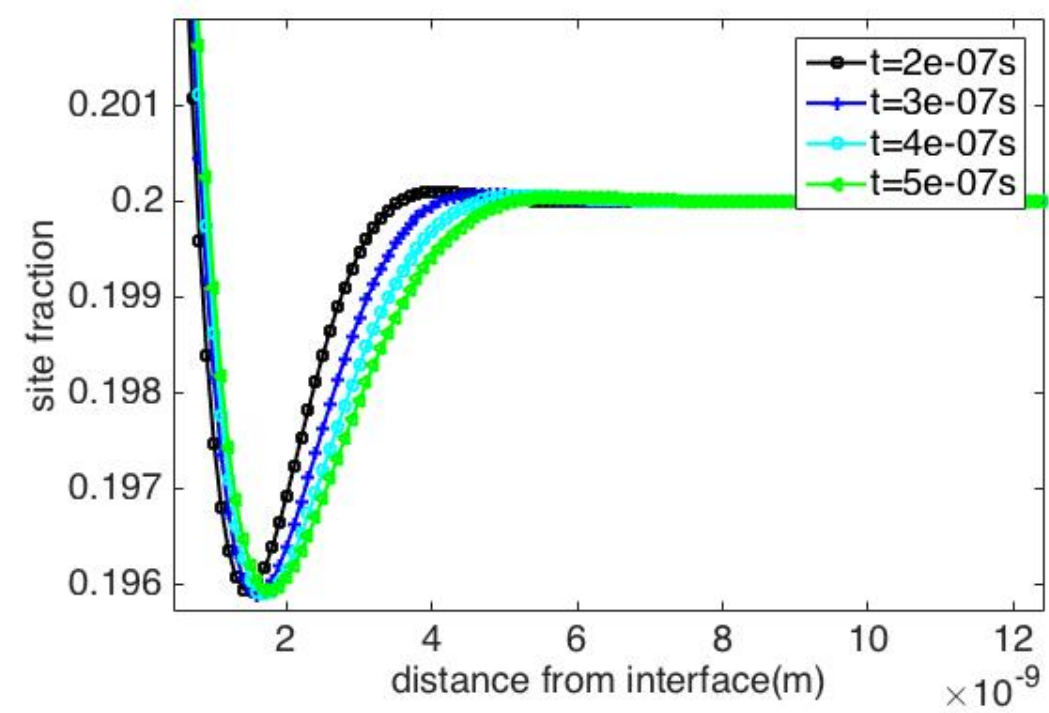

Figure 4.3: Dopant profile as a function of distance from the interface at $1300{ }^{\circ} \mathrm{C}$ and $20 \%$ dopant cation site fraction, after the second segregation zone begins to disappear

The extent of dopant profile change decreases over time, as the system reaches equilibrium: temporal changes on the time scales greater than $10^{-7} \mathrm{~s}$ are much smaller than those on the time scale of $10^{-8} \mathrm{~s}$. The cause of the rapid changes at the beginning of the 
simulation is the accumulation of oxygen vacancies at the interface and correspondingly high electrostatic driving force for dopant segregation; the segregation that occurs during the initial annealing period screens the core charge and weakens the driving force for further segregation. At this point, the main segregation zone near the interface gradually widens and the depth of dopant depletion decreases as shown in Figure 4.4. Here we also see the depletion zone thickness gradually extended from less than $2 \mathrm{~nm}$ to the around $10 \mathrm{~nm}$ at a time scale of $10^{-5} \mathrm{~s}$.

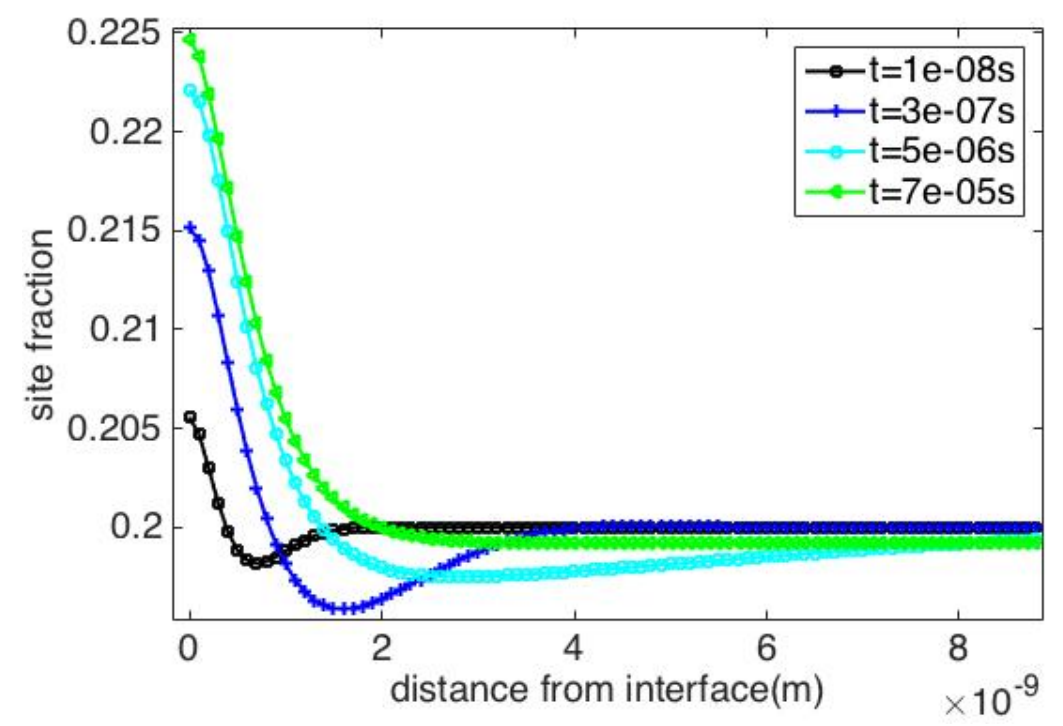

Figure 4.4: Dopant profile as a function of distance from the interface at $1300{ }^{\circ} \mathrm{C}$ and $20 \%$ dopant cation site fraction, between $10^{-7}$ and $10^{-5} \mathrm{~s}$

Figure 4.5 shows the dopant profile between 0.0001 and $0.017 \mathrm{~s}$. The width of the depletion zone extends to tens of nanometers before gradually disappearing. In less than 0.1 $\mathrm{s}$, the dopant distribution reaches equilibrium as seen by the overlapping profiles in Figure 4.6 . 


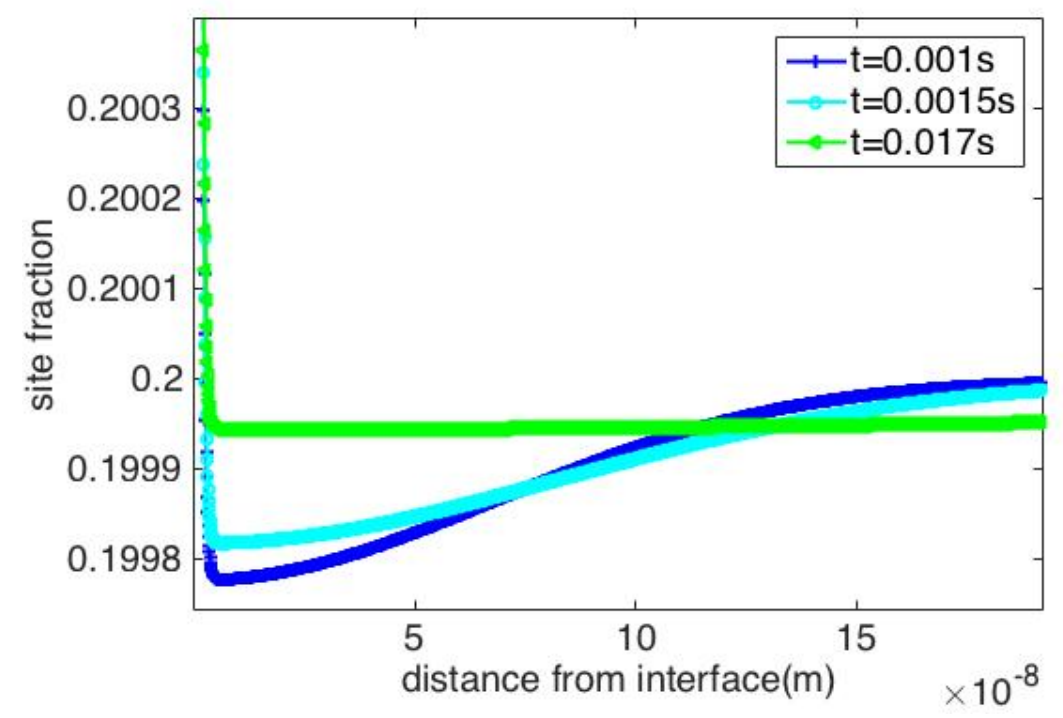

Figure 4.5: Dopant profile as a function of distance from the interface at $1300{ }^{\circ} \mathrm{C}$ and $20 \%$ dopant cation site fraction, at $\mathrm{t}=0.001,0.0015$ and $0.017 \mathrm{~s}$

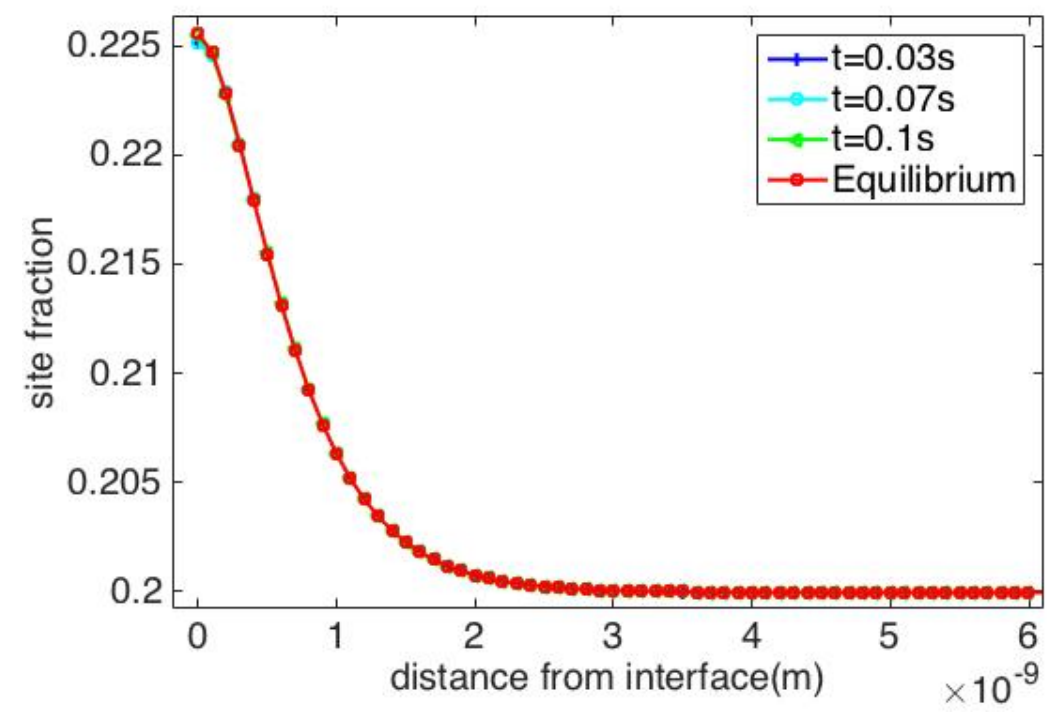

Figure 4.6: Dopant profile as a function of distance from the interface at $1300{ }^{\circ} \mathrm{C}$ and $20 \%$ dopant cation site fraction, at $\mathrm{t}=0.03,0.07,0.1 \mathrm{~s}$ and at equilibrium

Vacancies equilibrate at every time step with respect to the dopant due to their relatively high mobility. The vacancy profiles remain close to the profile at the overall system equilibrium as shown in Figure 4.7. Similar results were obtained for the electrostatic 
potential, as shown in Figure 4.8.

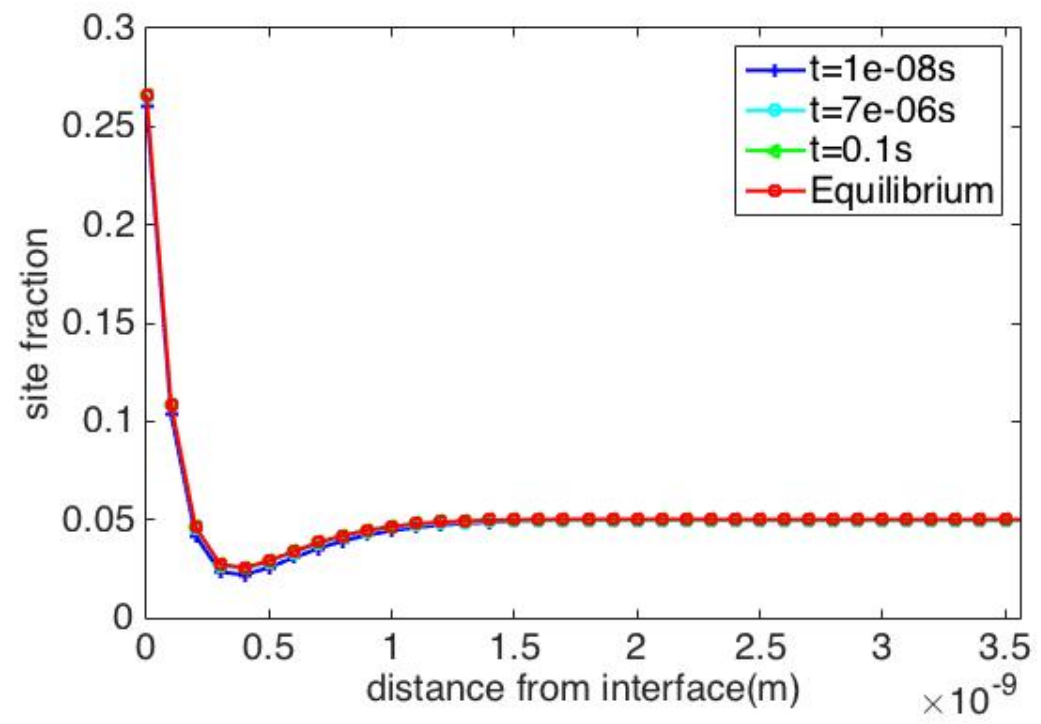

Figure 4.7: Vacancy site fraction as a function of distance from the interface at $1300{ }^{\circ} \mathrm{C}$ and $20 \%$ dopant cation site fraction

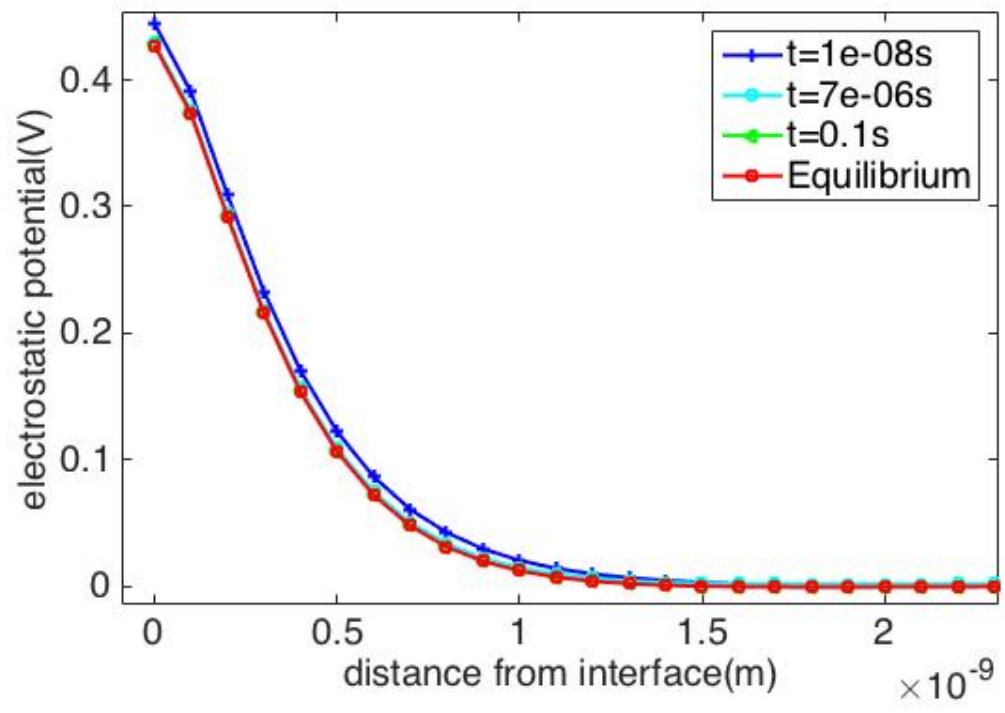

Figure 4.8: Electrostatic potential as a function of distance from the interface at $1300{ }^{\circ} \mathrm{C}$ and $20 \%$ dopant cation site fraction

For $1 \%$ dopant concentration, the dopant profile developed in a similar fashion to the more concentrated case presented above, but without the emergence of a second segregation 
zone. It takes less than $1 \mathrm{~s}$ for the dopant profile to approach equilibrium. For $0.1 \%$ dopant concentration, dopant segregation began with the same fast timescale as the more concentrated cases, followed with a shallow and wide depletion region. Long range effects were also observed at $0.1 \%$ dopant concentration, as the shallow depletion region extends on the order of tens of nanometers into the bulk prior to equilibration.

The model was also used to calculate equilibration times for GDC with $20 \%$ dopant concentrations when annealing under temperatures less than $1300^{\circ} \mathrm{C}$. Using $99.95 \%$ correspondence (in an $L^{2}$-norm sense) of the equilibrium profile in the first $10 \mathrm{~nm}$ region as the criterion for attaining equilibrium, Figure 4.9, shows the time to reach equilibrium for multiple temperatures. A quench temperature of the dopant profile of approximately $900{ }^{\circ} \mathrm{C}$ - at which it took more than 15 hrs. for dopant profile to approach equilibrium - can be deduced. Similar trend is expected for different doping levels because cation mobility quickly decreases, as temperature gets lower.

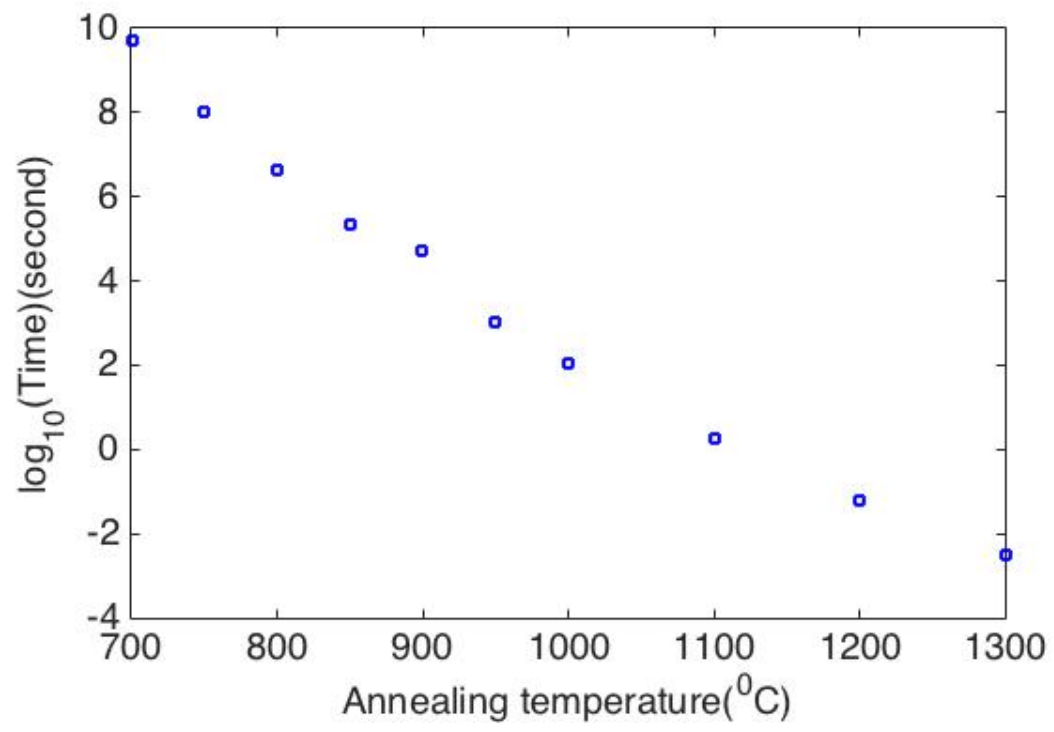

Figure 4.9: Time to equilibrium as a function of annealing temperature for GDC with $20 \%$ dopant concentration 


\section{CHAPTER 5}

\section{POISSON-CAHN MODEL PARAMETERIZATION}

\subsection{Modeling Grain Boundary Resistance of GDC}

In this study, the Poisson-Cahn theory is applied to $\mathrm{Ce}_{1-\mathrm{x}} \mathrm{Gd}_{\mathrm{x}} \mathrm{O}_{2-\mathrm{x} / 2}$ of different $\mathrm{Gd}$ dopant concentrations to predict the defect concentrations near GB. With these prediction results from the Poisson-Cahn model, the bulk and grain boundary resistances were calculated using the Nakayama-Martin conductivity model. A realistic case of a restricted equilibrium is considered: the acceptor-dopant profile is frozen-in from the quench temperature identified with the kinetic model, while the oxygen vacancies are mobile at all temperatures and are therefore instantly equilibrated at the measurement temperature.

In this study, the bulk and grain boundary conductivity data was used to perform a particle swarm optimization in search of parameter setting to realize a good fit between the model predictions and the experimental data. The list of fitted parameters are summarized in Table 5.1. In Figure 5.1, literature data for measured isothermal bulk and total conductivities of the $\mathrm{CeO}_{2}-\mathrm{Gd}_{2} \mathrm{O}_{3}$ system are compared with the results of Poisson-Cahn calculations. The PC predictions approximate the conductivity data for both the bulk and the total conductivity (considering the grain boundary effect) for systems with a wide range of dopant concentrations. The sudden drop in total conductivity as dopant concentration decreases, is also successfully predicted. 


\begin{tabular}{lll} 
Param. & Value & Indication \\
\hline$n_{0}$ & $1.89 \times 10^{-5} \mathrm{~mol} / \mathrm{m}^{2}$ & interfacial site density for vacancies \\
$f_{\mathrm{v}}$ & $0.10 \mathrm{eV}$ & vacancy self-interaction energy \\
$f_{0}$ & $-4.70 \mathrm{eV}$ & vacancy segregation energy \\
$f_{\mathrm{y}}$ & $0.44 \mathrm{eV}$ & dopant self-interaction \\
$f_{\mathrm{yv}}$ & $-0.06 \mathrm{eV}[12]$ & dopant-vacancy interaction \\
$c_{\mathrm{v}}$ & $1.05 \mathrm{eV}-\mathrm{nm}^{2}$ & vacancy gradient energy coefficient \\
$c_{\mathrm{d}}$ & $2.30 \mathrm{eV}-\mathrm{nm}^{2}$ & dopant gradient energy coefficient \\
\hline
\end{tabular}

Table 5.1: Fitted parameter values from particle swarm optimization
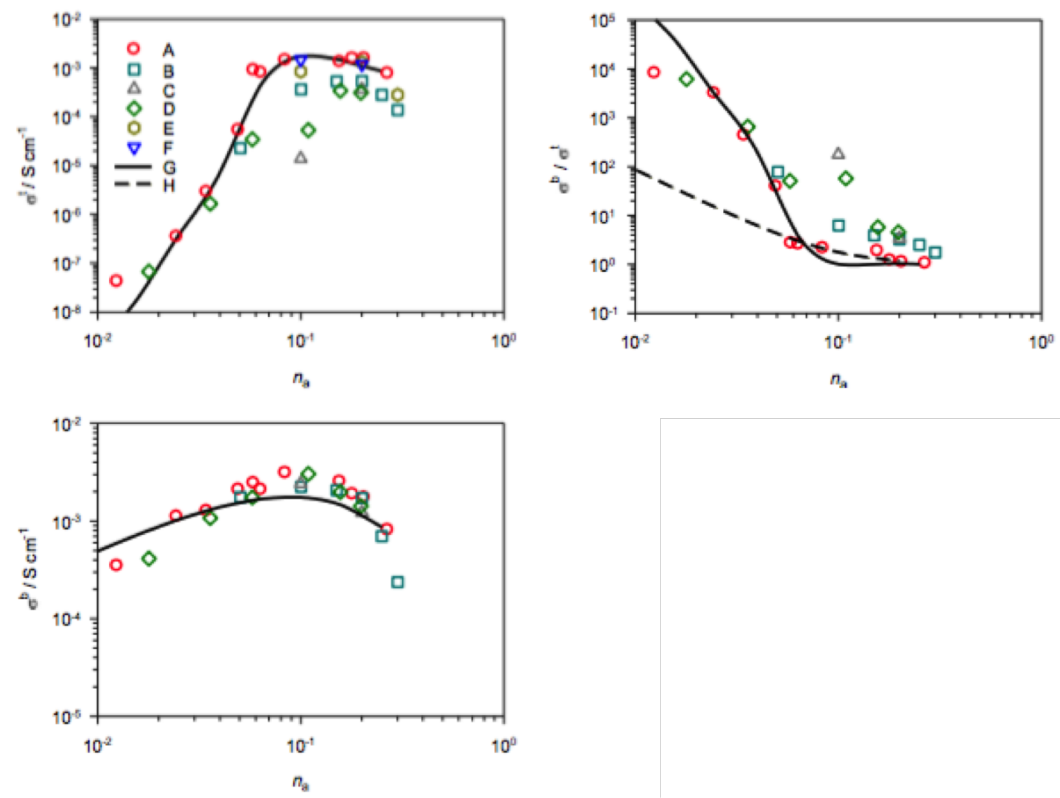

Figure 5.1: Conductivity $\sigma$ of the $\mathrm{CeO}_{2}-\mathrm{Gd}_{2} \mathrm{O}_{3}$ system as a function of $n_{a}$ at $\mathrm{T}=713 \mathrm{~K}$

In Figure 5.1, $n_{a}$ denotes Gd site fraction. Data has been taken from experiment and extrapolated or interpolated where necessary:(A) Tschope et al. [22],(B) Tianshu et al. [118],(C) Ralph et al. [119], (D) Avila-Paredes et al. [120], (E) Kudo and Obayashi [121], (F) Kharton et al. [122], (G) Poisson-Cahn analysis, (H) Poisson-Cahn with interaction energies 
set to 0 .

When we compare the dopant equilibrium profile from the Poisson-Cahn calculations with the results from the Gouy-Chapman theory, Figure 5.2 shows a series of selective comparisons. PC results showed segregation layers of several nanometers especially at high dopant concentrations. As for the characteristic length, PC results reasonably matched the GC results at low dopant concentrations while diverged from GC results expectedly at higher dopant concentrations owing to the limitations in applying GC to highly concentrated systems as in Figure 5.3. Similar trends are obtained from the space-charge potential, where PC predictions stayed close to the case of GC and MS and started to diverge as dopant concentration increases.
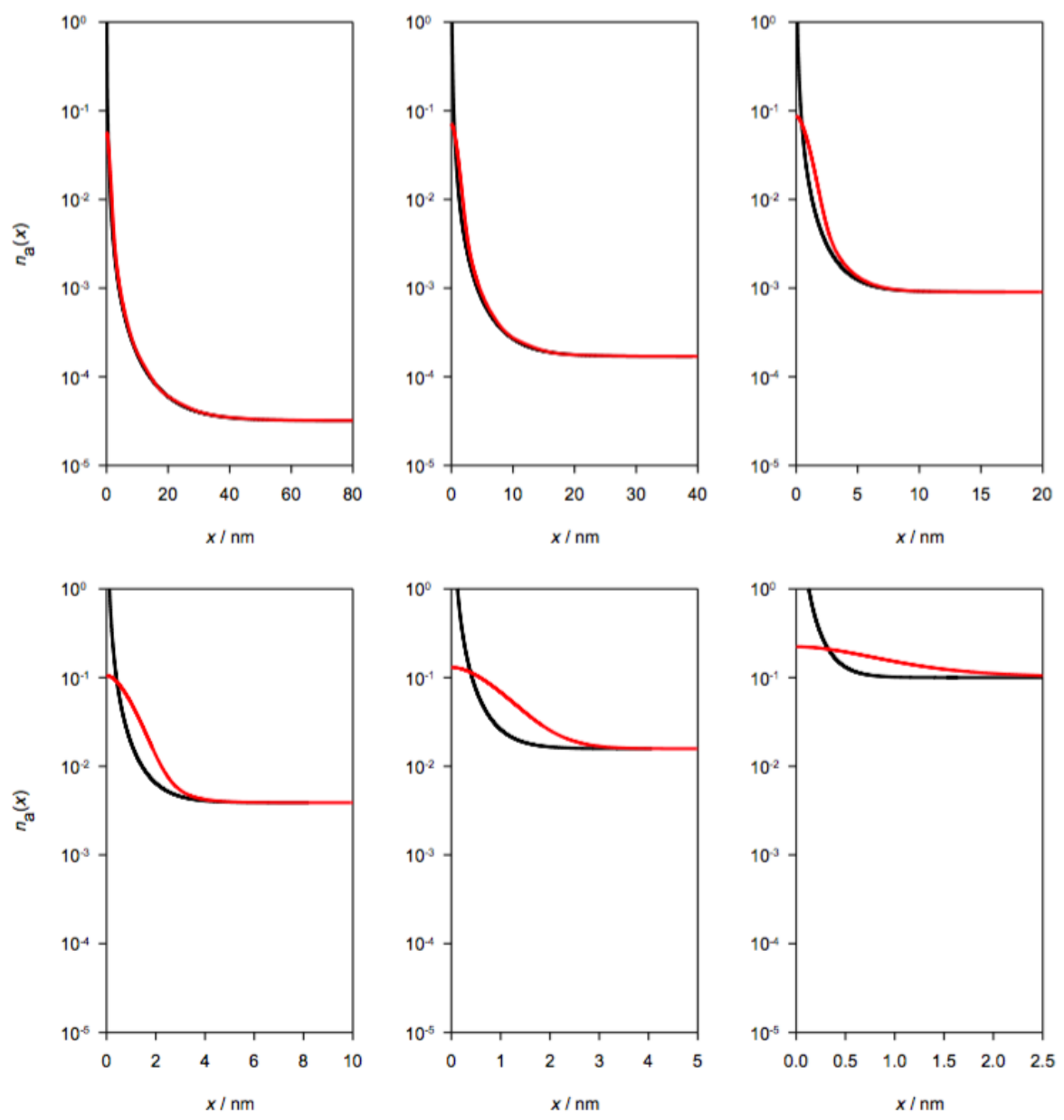

Figure 5.2: Dopant concentration profiles, $n_{a}(\mathrm{x})$, calculated from Poisson-Cahn theory(red) and from Gouy-Chapman theory(black) for six values dopant concentrations at $\mathrm{T}=1223 \mathrm{~K}$. 


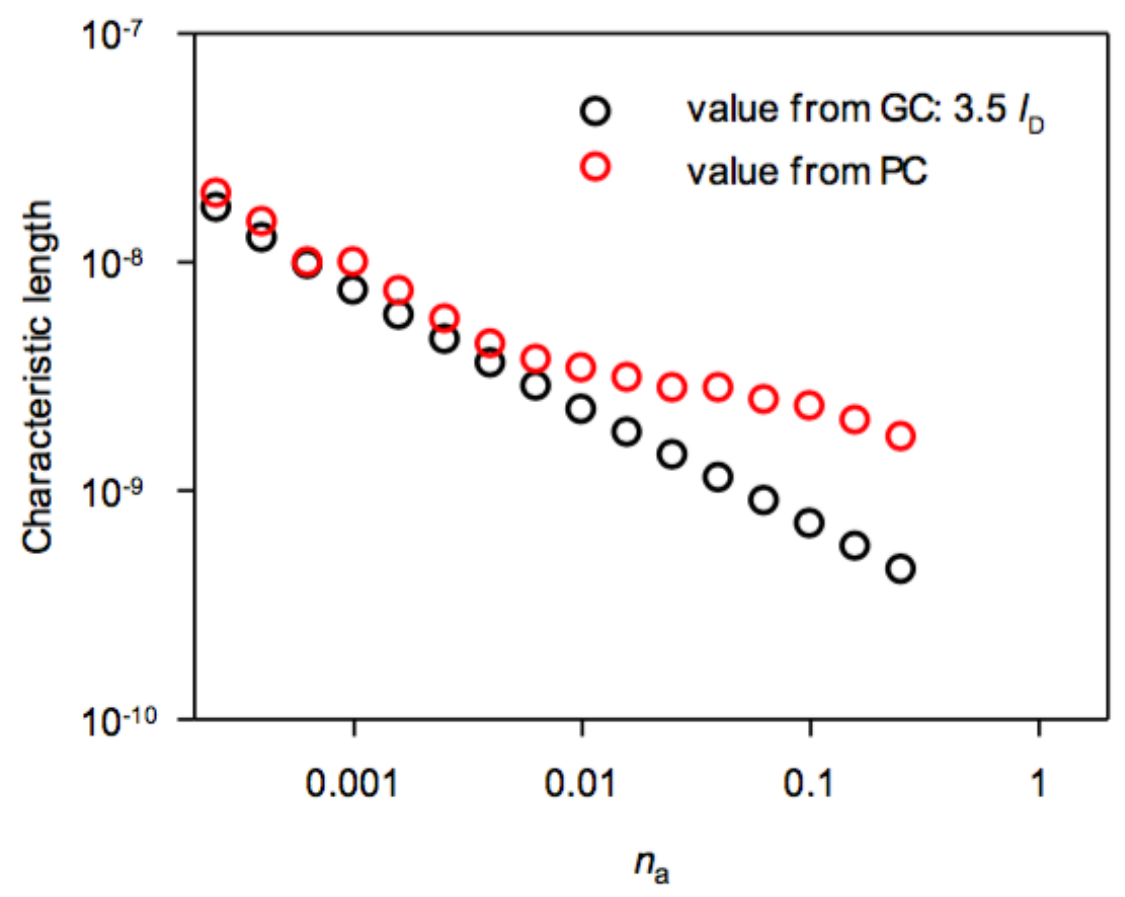

Figure 5.3: Extent of space-charge layer calculated from Poisson-Cahn theory. Compared with $3.5 l_{D}$. Extracted from $\varphi(x)=0.0078 \varphi_{0}$

\subsection{Modeling Grain Boundary Composition in NDC}

In this section, the Poisson-Cahn theory is applied to the Nd-doped ceria system with two different dopant concentrations (10 cat\% and 30 cat\%) to reproduce the experimental results of grain boundary compositions measured by atom probe tomography (APT). With the two-step equilibration strategy, Bayesian calibration was used to estimate the posterior parameter distributions for these solid solutions. The Poisson-Cahn model was shown to be able to quantitatively reproduce the experimental results.

The experiment was conducted by Diercks et al. and the results were published in 2016 [21]. In the experiment, samples of $\mathrm{Nd}_{0.10} \mathrm{Ce}_{0.90} \mathrm{O}_{2-\sigma}$ (NDC10) and $\mathrm{Nd}_{0.30} \mathrm{Ce}_{0.70} \mathrm{O}_{2-\sigma}$ (NDC30) were prepared in air through conventional solid state reactions. X-ray diffraction (XRD) was used to verify dopant incorporation and homogeneous phase formation after calcination. The final pellets were sintered in air at $1600^{\circ} \mathrm{C}$ for $10 \mathrm{~h}$. Specimens for APT 
were prepared using a site-specific life-out method targeting grain boundaries in an FEI Helios 600i DualBeam focused ion beam/scanning electron microscope (FIB/SEM). APT analysis was accomplished in a Cameca LEAP 4000X Si using a $40 \mathrm{~K}$ base temperature, 0.1-0.4 pJ nominal laser pulse energy, a pulse repetition rate of $625 \mathrm{kHz}$ and a detection rate of 3 ions per 1000 pulses (0.3\%). Specimen reconstruction were generated using Cameca's IVAS 3.6.4 analysis software. In this data source, the lower dopant concentration (10 cat\%) is selected as it represents compositions typical for electrolytes in solid oxide fuel cells. The higher dopant concentration is selected as a comparison in the high dopant range while below the concentration that induces a disorder-order phase transformation. Significant differences between the compounds were observed experimentally and the Poisson-Cahn model was shown to be a powerful tool to reproduce the composition profiles for systems of different concentrations.

The compositions of oxygen, cation and impurities present were simultaneously quantified. To directly compare with these experimental measurements, predictions in terms of site fraction of dopant and vacancies are converted into elemental compositions. Let $n_{o}$, $n_{v}, n_{d}, n_{c}$ be the site densities of oxygen ions, oxygen vacancies, dopants and cerium ions respectively, and $r_{d}, r_{o}$ be the compositional percentage of dopants and oxygen respectively. Ignoring the small percentage of impurities present, we can establish the following relationship.

$$
\begin{aligned}
& \frac{n_{o}}{n_{o}+n_{d}+n_{c}}=r_{o} \\
& \frac{n_{d}}{n_{o}+n_{d}+n_{c}}=r_{d}
\end{aligned}
$$

Considering the fluorite structure of the doped system, the relationship below stands.

$$
\begin{aligned}
& n_{v}+n_{o}=n_{o s} \\
& n_{c}+n_{d}=n_{c s}
\end{aligned}
$$


Where $n_{o s}$ and $n_{c s}$ are anion and cation site density in the cubic fluorite structure, respectively.

$$
\begin{gathered}
n_{c s}=\frac{4}{N_{A} * \lambda^{3}} \\
n_{o s}=2 n_{c s}=\frac{8}{N_{A} * \lambda^{3}}
\end{gathered}
$$

Therefore, site fractions of dopant and vacancies, denoted by y and v respectively, are linked with their corresponding site densities through the equations below.

$$
\begin{gathered}
y=\frac{n_{d}}{n_{c s}} \\
v=\frac{n_{v}}{n_{o s}}
\end{gathered}
$$

Combining equations 5.1 - 5.8, the relationship between site fractions of of dopant and vacancies and composition percentages of dopant and oxygen can be obtained.

$$
\begin{aligned}
& r_{d}=\frac{y \cdot n_{c s}}{n_{o s}-v \cdot n_{o s}+n_{c s}} \\
& r_{o}=\frac{n_{o s}-v \cdot n_{o s}}{n_{o s}-v \cdot n_{o s}+n_{c s}}
\end{aligned}
$$

The above conversions were built into the Poisson-Cahn model so that model predictions can be directly compared with compositional percentage data measured by APT. Figure 5.4 displays compositional profiels of $\mathrm{O}, \mathrm{Ce}$, and $\mathrm{Nb}$ averaged over dozens of individual grain boundaries. 

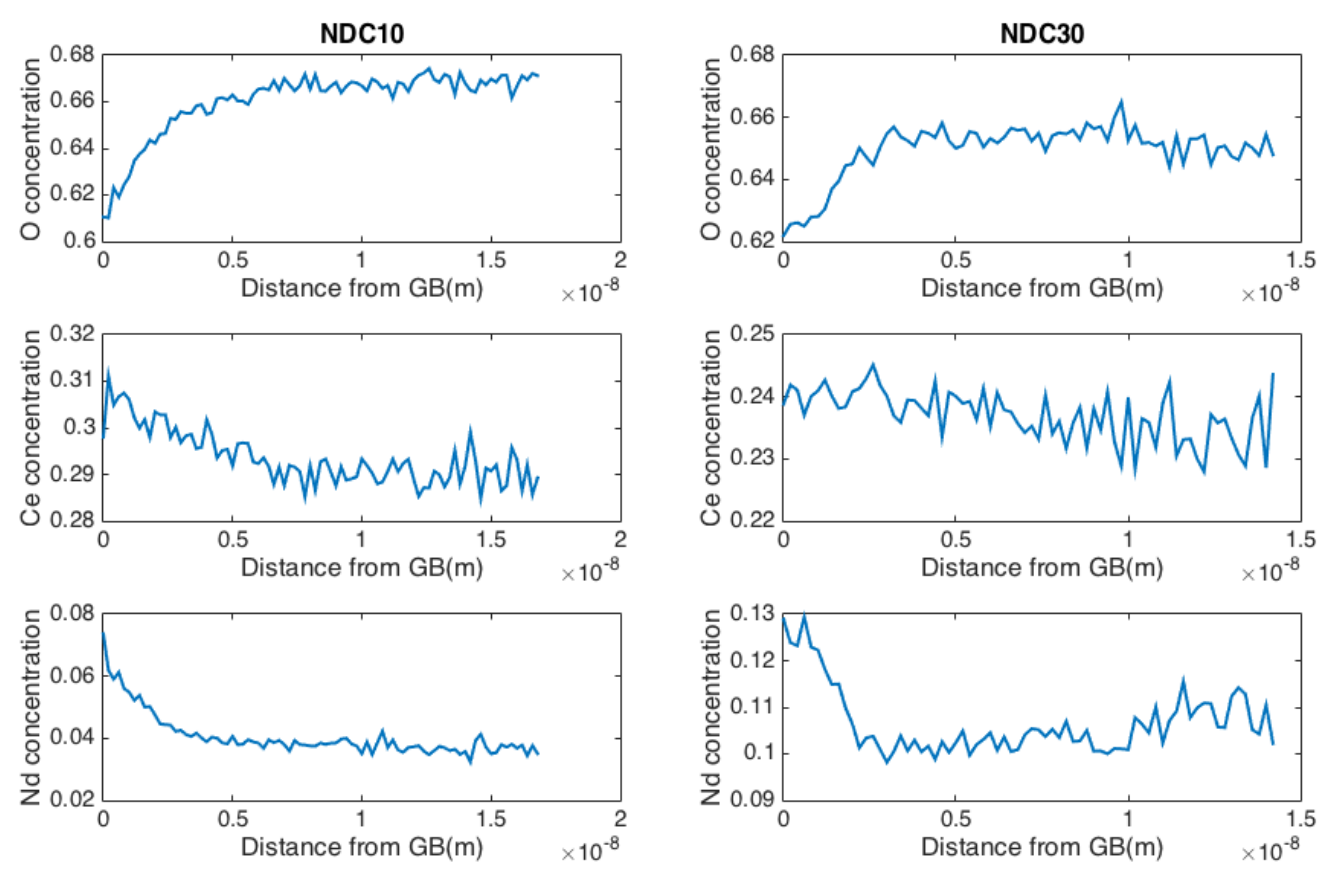

Figure 5.4: Raw APT data for 10\% (left) and 30\% samples

Under a Bayesian calibration framework, posterior distributions of the model parameters were estimated for NDC10 and NDC30 separately using the same prior distributions. The Poisson-Cahn model here treat the more realistic case of restricted equilibrium where dopant ions were equilibrated at $950^{\circ} \mathrm{C}$ ( a temperature level where equilibration time starts to exceed cooling rate of the experiment) and the equilibration temperature for vacancies and polarons were a calibration parameter. Table 5.2 provides a full list of calibration parameters and their abbreviation forms adopted in the model. 


\begin{tabular}{ll} 
Param. & Indication \\
\hline$T 2$ & equilibration temperature for vacancies and electrons \\
$n_{0}$ & interfacial site density for vacancies \\
$f_{\mathrm{yy}}$ & dopant self-interaction \\
$f_{\mathrm{vv}}$ & vacancy self-interaction energy \\
$f_{\mathrm{qq}}$ & electron self-interaction energy \\
$f_{\mathrm{yv}}$ & dopant-vacancy interaction \\
$f_{\mathrm{yq}}$ & dopant-electron interaction \\
$f_{\mathrm{vq}}$ & vacancy-electron interaction \\
$c_{\mathrm{v}}$ & vacancy gradient energy coefficient \\
$c_{\mathrm{d}}$ & dopant gradient energy coefficient \\
$c_{\mathrm{q}}$ & electron gradient energy coefficient \\
$f_{\mathrm{qs}}$ & electron surface affinity \\
$f_{\mathrm{ds}}$ & dopant surface affinity \\
$H r$ & reaction enthalpy for the reduction of ceria \\
$S r$ & reaction entropy for the reduction of ceria \\
\hline
\end{tabular}

Table 5.2: Model parameters for Bayesian calibration

A number of MCMC steps have been executed before the calibration converged, meaning the posterior distributions of model parameters are obtained. The logarithm values of the likelihood as a function of MCMC steps is shown in Figure 5.5. At the start of the calibration, the likelihood value is relatively small. This value continued to increase while the calibration is running. Upon burning-in, value of the likelihood bounced within a high-value region, indicating that the convergency of the routine.

Figure 5.6 and 5.7 shows the selective parameter traces - the value of parameter as a function of MCMC steps. Typically, from one point in the parameter spaces of the 


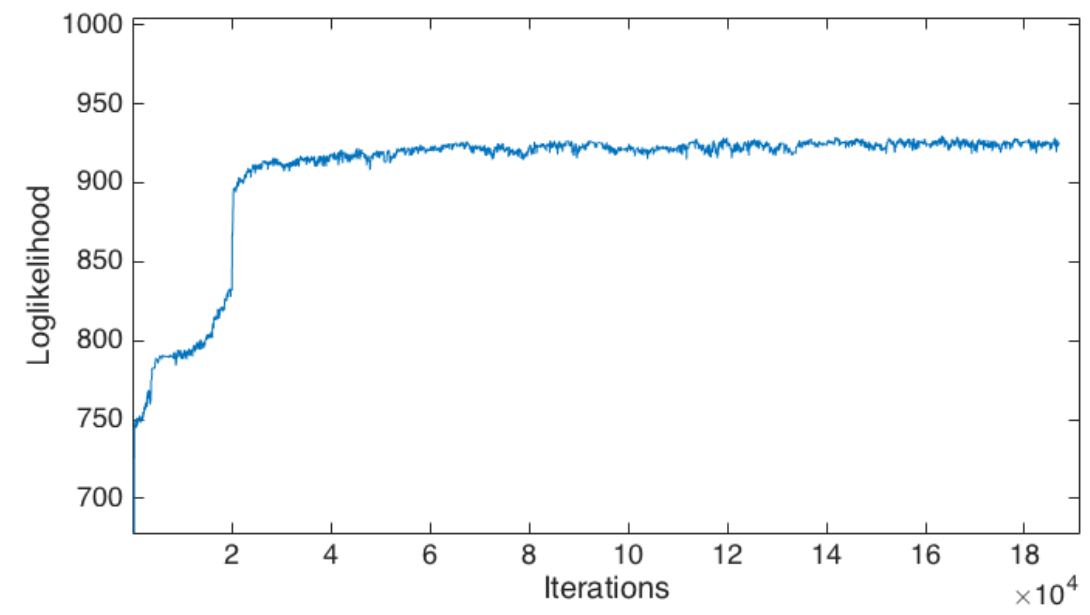

Figure 5.5: Log of Likelihood for model calibration with NDC10

prior distributions, the MCMC routine explores the predefined parameter spaces within the bounds before settling down at the parameter posterior distributions. After burning-in is achieved, the parameter traces changes within a relatively small region and the acceptance rate of the calibration becomes stable.
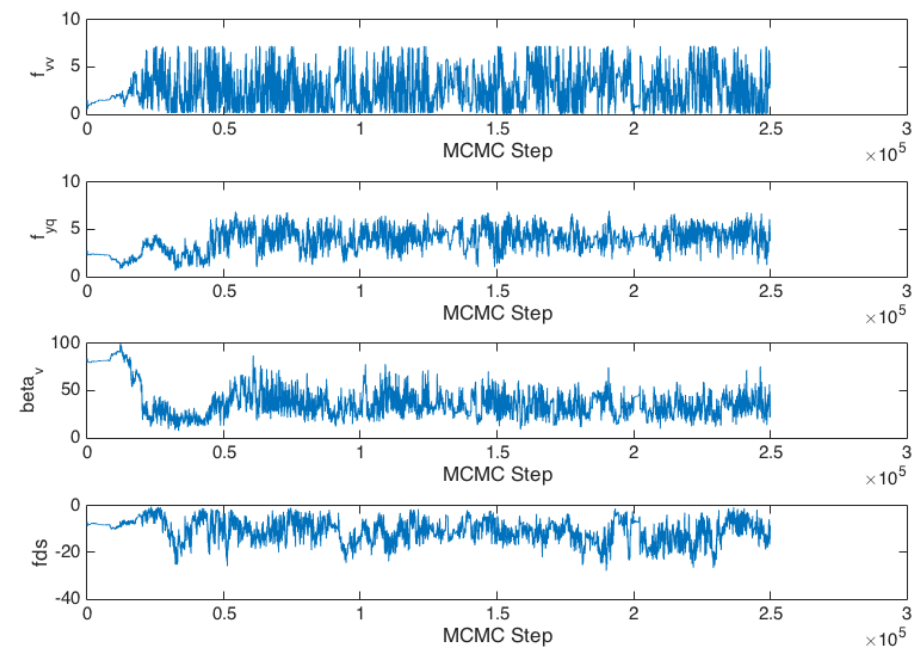

Figure 5.6: Selected parameters vs. MCMC steps for calibration of NDC10 

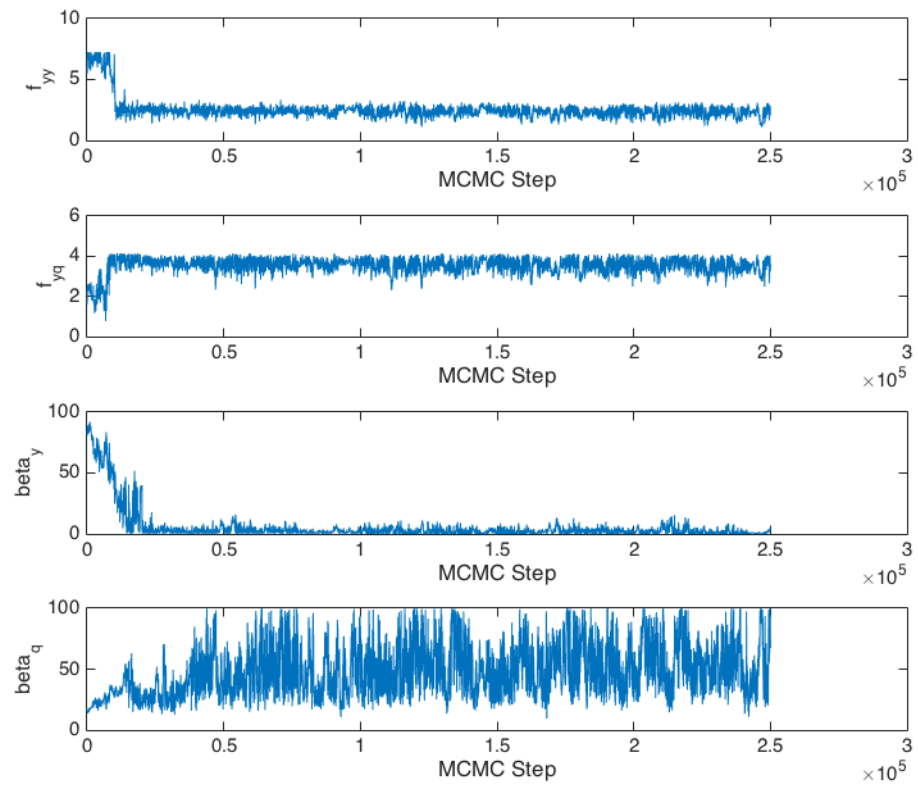

Figure 5.7: Selected parameters vs. MCMC steps for calibration of NDC30

For both NDC10 and NDC30, 250000 MCMC steps were acquired during the calibration and 70000 samples were cut out for burn-in, leaving 180000 samples in the posterior. Figures 5.6 and 5.7 show selected parameters as a function of MCMC step for NDC10 and NDC30. The figures show that equilibration/burn-in was achieved after 70000 samples for both datasets. Student's t-test results reveal that all 15 estimated parameter means fell within the preferred interval for the calibration of NDC10 data while three parameters exceeded the interval for NDC30. Those three parameters were $f_{\mathrm{vv}}( \pm 5.8 \%), \beta_{\mathrm{vv}}( \pm 6.8 \%)$ and $f_{\text {ds }}( \pm 6.8 \%)$.

Upon convergence of the calibration, distributions of the parameters are shown in the table below. Results show consistent posterior distributions of NDC10 and NDC 30 for parameters including $T 2, n_{\mathrm{os}}, f_{\mathrm{qq}}, f_{\mathrm{qs}}, H r$, and $S r$. As for defect interaction energies including $f_{\mathrm{yy}}, f_{\mathrm{vv}}, f_{\mathrm{yv}}, f_{\mathrm{yq}}$ and $f_{\mathrm{vq}}$, the posterior distributions of NDC10 are broader than those of NDC30 and the distribution average of each parameter is in general higher for NDC10 compared with that of NDC30. Similar relationship was also revealed when comparing 
posteriors of gradient energy terms including $c_{\mathrm{v}}, c_{\mathrm{y}}$ and $c_{\mathrm{q}}$ for NDC10 and NDC30. These findings indicate that defect interactions and the gradient effects are more profound for NDC10 than NDC30, which might be linked to the fact of possible defect reordering and phase transformation that takes place upon increasing dopant concentration up to a certain level.

Table 5.3: Posterior distributions for NDC10 and NDC30

\begin{tabular}{lllll}
\hline \multirow{2}{*}{ parameter } & \multicolumn{2}{c}{$10 \%$} & \multicolumn{2}{c}{$30 \%$} \\
& Mean & Std & Mean & Std \\
\hline$T_{2}(\mathrm{~K})$ & 1325 & 121.0 & 1132 & 47.4 \\
$n_{\mathrm{os}} \times 10^{5}\left(\mathrm{~mol} / \mathrm{m}^{2}\right)$ & 3.5 & 0.85 & 1.6 & 0.5 \\
$f_{\mathrm{yy}}(\mathrm{eV})$ & 57.5 & 10.2 & 22.7 & 3.1 \\
$f_{\mathrm{vv}}(\mathrm{eV})$ & 26.8 & 17.2 & 5.7 & 4.8 \\
$f_{\mathrm{qq}}(\mathrm{eV})$ & 33.3 & 6.0 & 21.1 & 4.4 \\
$f_{\mathrm{yv}}(\mathrm{eV})$ & 35.3 & 16.7 & 35.9 & 3.4 \\
$f_{\mathrm{yq}}(\mathrm{eV})$ & 40.6 & 9.2 & 34.2 & 2.7 \\
$f_{\mathrm{vq}}(\mathrm{eV})$ & 54.3 & 10.4 & 34.7 & 4.3 \\
$c_{\mathrm{v}}\left(\mathrm{eV}-\mathrm{nm}{ }^{2}\right)$ & 280.0 & 80.5 & 120.4 & 14.4 \\
$c_{\mathrm{y}}\left(\mathrm{eV}-\mathrm{nm}{ }^{2}\right)$ & 380.1 & 177.8 & 8.8 & 8.1 \\
$c_{\mathrm{q}}\left(\mathrm{ev}-\mathrm{nm}{ }^{2}\right)$ & 339.2 & 107.9 & 206.2 & 74.8 \\
$f_{\mathrm{qs}}(\mathrm{eV})$ & -48.0 & 8.5 & -44.6 & 10.9 \\
$f_{\mathrm{ds}}(\mathrm{eV})$ & -10.9 & 4.5 & -0.6 & 0.5 \\
$H_{\mathrm{r}}(\mathrm{eV})$ & 2.6 & 0.2 & 2.6 & 0.1 \\
$S_{\mathrm{r}}\left(\mathrm{J} / \mathrm{mol}^{2} \mathrm{~K}\right)$ & 108.1 & 14.8 & 109.1 & 11.8 \\
\hline & & & &
\end{tabular}

Figure 5.8 and 5.9 show coverages of the experimental data by overlaying on top 50 model realizations calculated from randomly selected parameter sets for NDC10 and NDC30 respectively. Since the anion concentration reported by the experiment may involve a shift 
due to the fact of lacking electric neutrality, the anion concentration calculated from the calibration model is shifted accordingly considering the discrepancy of average bulk anion concentration from the experiment and each calibration. The black curve is the elemental composition as a function of distance from the grain boundary. The blue curves are randomly selected model results. The experimental data is well-covered by the calibration results except for the bulk part which is due to the fact that the employed model assumes uniform concentration in the bulk while there is inevitably experimental error in the actual measurement.
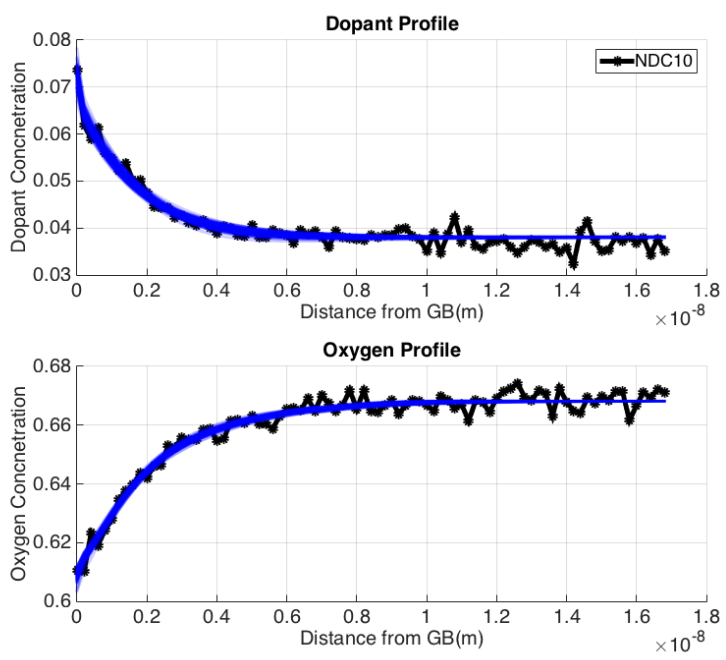

Figure 5.8: Calibration results for Models of NDC10 

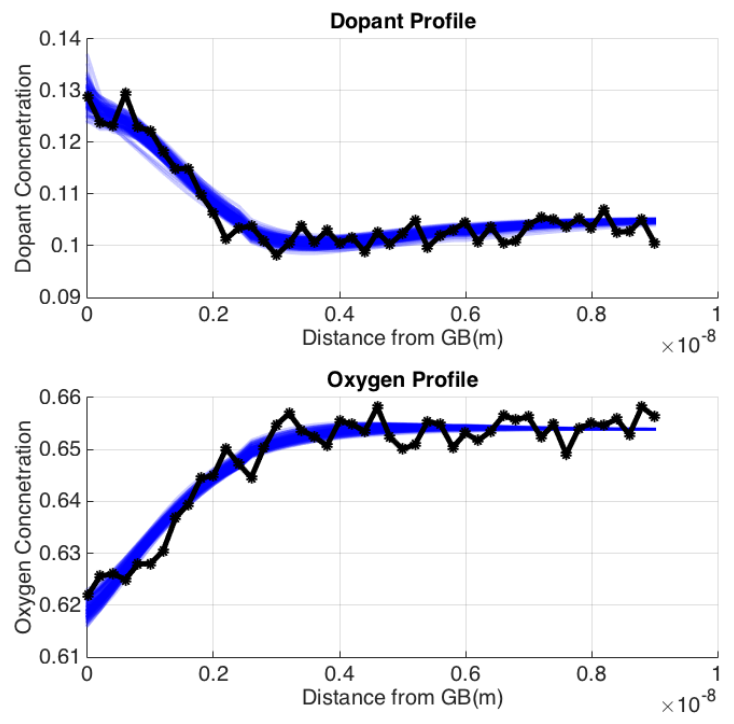

Figure 5.9: Calibration results for Models of NDC30

The model developed in this study has been shown to be able to replicate defect coaccumulation near the grain boundaries for concentrated systems, as represented by NDC10 and NDC30. The posterior results of $\mathrm{Hr}$, and $\mathrm{Sr}$ are consistent for these two systems as expected, since they are common values associated with the reduction of ceria in both systems. The mean values of the posterior parameter distributions of these systems are mostly consistent in terms of defect interaction parameters including $f_{\mathrm{qq}}, f_{\mathrm{yv}}, f_{\mathrm{yq}}$ and $f_{\mathrm{vq}}$, indicating similar defect interaction effects associated with these terms. The mean values of the posterior distributions of $f_{\mathrm{yy}}, f_{\mathrm{vv}}$ and $f_{\mathrm{vq}}$ are considerably higher for NDC10 and NDC30, indicating that the dopant and vacancy self-interactions, as well as the interaction between vacancies and electrons, are stronger in NDC10. The means values of gradient energy coefficients for the three defect species are also higher for NDC10, showing that the extent of gradient energy effects are more profound in the NDC10 system. The number of vacancy segregation sites are also higher for NDC10, indicating the stronger tendency for oxygen species to segregate to the grain boundary. The surface affinity of electrons are similar while that of the dopant are higher for NDC10, showing that it might be easier for dopant ions to segregate towards the interface in NDC10. Despite the fact that both NDC10 and 
NDC30 are concentrated systems, these results of these differed posterior distributions show that the underlying mechanisms of gradient energy effects and certain defect interactions might be different for these systems. Possibly, when the concentration increases from $10 \%$ to $30 \%$, defect reordering and phase changes might be present. The calibrated equilibration temperature for NDC10 are higher than for NDC30. For NDC10, this temperature is close to the quenching temperature of the dopant, which resembles previous studies where the Gouy-Chapman form was adopted: dopant and vacancies are equilibrated at the same temperature. [30, 31, 32, 33] For NDC30, the vacancy equilibration temperature are lower than the quenching temperature of the dopant, as expected, because of the different mobilities of these species. This, however, indicates that equilibration temperatures might differ depending on the dopant concentrations.

Parameter correlations were also gauged for each system and the top 3 correlated parameter pairs were presented here. In Figure 5.10 and Figure 5.11, the posterior distributions of the selected parameter pairs are presented via bivariate scatter plots. The intensity of the color in these figures is related to the number of parameters pertaining to the corresponding region, that is, the parameters are more likely to fall into the yellow color region compared to the blue color region. For NDC10, the correlations between $f_{\mathrm{vq}}$ and $f_{\mathrm{qq}}, f_{\mathrm{yq}}$ and $f_{\mathrm{yv}}$, and $\beta_{\mathrm{q}}$ and $n_{\mathrm{os}}$ were $0.78,0.77$ and 0.76 , respectively. For NDC30, the correlations between $f_{\mathrm{yv}}$ and $f_{\mathrm{yy}}, f_{\mathrm{yq}}$ and $f_{\mathrm{yy}}$, and $f_{\mathrm{vq}}$ and $f_{\mathrm{yq}}$ were $0.80,0.76$ and 0.73 , respectively. The interactions between species are shown to be correlated. The number of oxygen sites at the surface is also shown to be highly correlated with the gradient energy coefficients of electrons since the positively charged vacancies attract the electrons. This correlation is also relatively high for NDC30, with a value of 0.62 . 


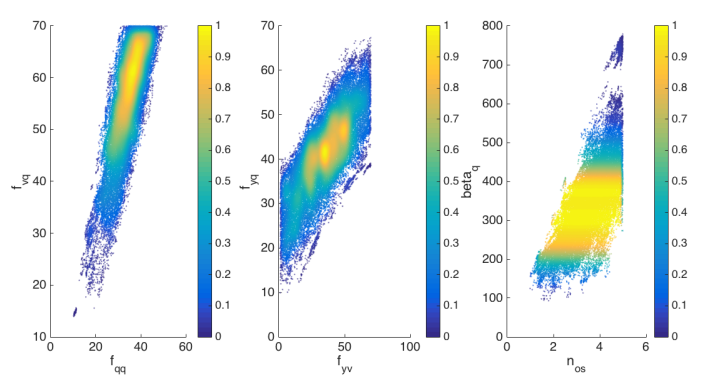

Figure 5.10: Bivariate scatter plots for Models of NDC10

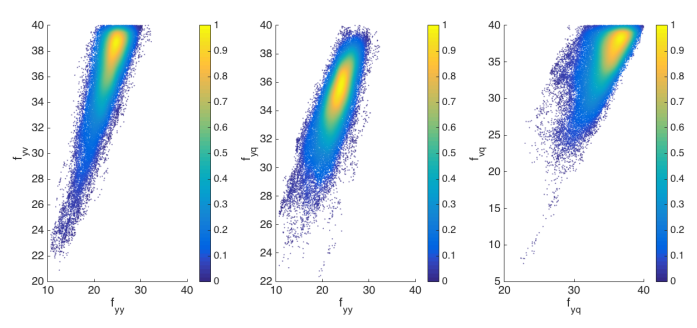

Figure 5.11: Bivariate scatter plots for Models of NDC30

Despite the capability of the developed model in replicating the co-accumulation phenomenon, differences in the parameter configurations for multiple material systems in this study demand further development of this Poisson-Cahn model. The functional forms of the contributions from defect interactions and gradient energy affects the parameter spaces of model parameters, which might be unified across multiple material systems. Future research following this direction is then focused on unifying the parameter space under the PoissonCahn theoretical framework through incorporating more flexible statistical functional forms for the components of the free energy functional, as presented in Chapter 6. 


\section{CHAPTER 6}

\section{DEVELOPING A UNIFIED POISSON CAHN FRAMEWORK WITH BSS-ANOVA METHOD}

For the ionic solid electrolyte material systems under study, the gradient and interaction energies are related and the exact relationship remains unknown. Therefore, from a modeling perspective, the best approach is to propose a general schema that covers a spectrum of potential functional relationships and allow the experimental data to reveal the underlying relationships through Bayesian calibration. The potential functional relationships are represented as discrepancy functions in the free energy functional of the material system, which represents the general framework of the unified Poisson-Cahn theory.

\subsection{Derivation of a Unified Poisson-Cahn Framework using BSS-ANOVA Dis- crepancy Functions}

To begin with, the model formulation and derivations in this section treat a simplified case of a pair of defect species, dopant ions and oxygen vacancies. Augmenting the development found in [12] with discrepancy functions, the free energy functional of the material system can be represented

$$
\begin{aligned}
\Omega[y, v, q, \varphi ; T]= & n_{\mathrm{o}} f_{\mathrm{o}} v(0)+\frac{1}{2} n_{\mathrm{o}} f_{\mathrm{ds}} y(0)+\int_{0}^{L}\left[\delta\left(v, y,\left(\frac{d v}{d x}\right)^{2},\left(\frac{d y}{d x}\right)^{2}\right)\right. \\
& +n_{\mathrm{cs}} R T[y \log (y)+(1-y) \log (1-y)] \\
& +n_{\mathrm{os}} R T[v \log (v)+(1-v) \log (1-v)] \\
& \left.-\frac{1}{2} \varepsilon_{r} \varepsilon_{0}\left(\frac{d \varphi}{d x}\right)^{2}+F \varphi\left(2 n_{o s} v-n_{c s} y\right)\right] d x
\end{aligned}
$$


Where $\delta\left(v, y,\left(\frac{d v}{d x}\right)^{2},\left(\frac{d y}{d x}\right)^{2}\right)$ represents the total sum of the discrepancy functions related with excess free energy and gradient energy:

$$
\begin{aligned}
\delta\left(v, y,\left(\frac{d v}{d x}\right)^{2},\left(\frac{d y}{d x}\right)^{2}\right)= & \text { Const }+\sum_{i} \beta_{\mathrm{v} i} \phi_{i}(v)+\sum_{i} \beta_{\mathrm{y} i} \phi_{i}(y)+\sum_{i} \beta_{\mathrm{vy} i} \phi_{i}(v) \phi_{i}(y) \\
& \beta_{\mathrm{v}^{\prime} i}^{0}\left(\frac{d v}{d x}\right)^{2}+\beta_{\mathrm{y}^{\prime} i}^{0}\left(\frac{d y}{d x}\right)^{2}+\sum_{i} \beta_{\mathrm{v}^{\prime} i} \phi_{i}(v)\left(\frac{d v}{d x}\right)^{2}+\sum_{i} \beta_{\mathrm{y}^{\prime} i} \phi_{i}(y)\left(\frac{d y}{d x}\right)^{2}
\end{aligned}
$$

where $\mathrm{i}$ indexes over the number of basis functions of the form in 2.1. A constant term Const is present in $\delta$ in order to make sure that the discrepancy functions corresponding to defect interactions are 0 when defect concentrations are 0 . The existence of this constant term doesn't affect the variational analysis part.

If we represent the sum of all original terms in the free energy with $\Omega_{0}$ except the sum of all discrepancy functions in Equation 6.1. Then the conceptual form of the system free energy becomes:

$$
\Omega[y, v, q, \phi ; T]=\Omega_{0}+\int_{0}^{L} \delta\left(v, y,\left(\frac{d v}{d x}\right)^{2},\left(\frac{d y}{d x}\right)^{2}\right) d x
$$

Minimization of the free energy functional is subject to the constraint of the conservation of mass in the system as shown in 6.4 and 6.5 .

$$
\begin{aligned}
& \int_{0}^{L}(v-\bar{v}) d x=0 \\
& \int_{0}^{L}(y-\bar{y}) d x=0
\end{aligned}
$$

where $\bar{y}$ and $\bar{v}$ are the average dopant and vacancy site fraction in the material. This constraints are added to the functional using lagrange multiplier $\lambda_{y}$ and $\lambda_{v}$.

The minimization starts with taking variational differentiation with respect to v. Let $v=f+\varepsilon \eta$, where $\eta(x)$ is an arbitrary function that is at least first-order differentiable and 
becomes zero at both endpoints of the domain. $\mathrm{f}$ is where the functional attains a minimum. Thus, the functional is minimized when $\varepsilon=0$. Taking the total derivative of $\Omega\left(v,\left(\frac{d v}{d x}\right)^{2}\right)$, where $v=f+\varepsilon \eta$ and $v^{\prime}=f^{\prime}+\varepsilon \eta^{\prime}$ are functions of $\varepsilon$ but $\mathrm{x}$ is not,

$$
\begin{aligned}
\frac{d \Omega}{d \varepsilon}= & \frac{\partial \Omega_{0}}{\partial v} \frac{d v}{d \varepsilon}+\frac{\partial \Omega_{0}}{\partial v^{\prime}} \frac{d v}{d \varepsilon}+\int_{0}^{L}\left[\sum_{i} \beta_{\mathrm{v} i} \phi_{i}^{\prime}(v) \eta+2 \beta_{\mathrm{v}^{\prime} i}^{0} \frac{d v}{d x} \eta^{\prime}+\sum_{i} \beta_{\mathrm{v}^{\prime} i} \phi_{i}^{\prime}(v)\left(\frac{d v}{d x}\right)^{2} \eta\right. \\
& \left.+\sum_{i} \beta_{\mathrm{v}^{\prime} i} \phi_{i}(v) 2 \frac{d v}{d x} \eta^{\prime}+\sum_{i} \beta_{\mathrm{vy} i} \phi_{i}^{\prime}(v) \phi_{i}(y) \eta+\lambda_{v} \eta\right] d x
\end{aligned}
$$

Applying integration by parts to the terms containing $\eta^{\prime}$ in the above equation and reorganizing terms related to $\eta$ yields:

$$
\begin{aligned}
\frac{d \Omega}{d \varepsilon}= & \frac{\partial \Omega_{0}}{\partial v} \frac{d v}{d \varepsilon}+\frac{\partial \Omega_{0}}{\partial v^{\prime}} \frac{d v}{d \varepsilon}+\int_{0}^{L}\left[\sum_{i} \beta_{\mathrm{v} i} \phi_{i}^{\prime}(v)+\sum_{i} \beta_{\mathrm{v}^{\prime} i} \phi_{i}^{\prime}(v)\left(\frac{d v}{d x}\right)^{2}\right. \\
& \left.-2 \beta_{\mathrm{v}^{\prime} i}^{0} \frac{d^{2} v}{d x^{2}}-\sum_{i} \beta_{\mathrm{v}^{\prime} i} \phi_{i}^{\prime}(v) 2 \frac{d v}{d x}-\sum_{i} \beta_{\mathrm{v}^{\prime} i} \phi_{i}(v) 2 \frac{d^{2} v}{d x^{2}}+\sum_{i} \beta_{\mathrm{vy}^{i}} \phi_{i}^{\prime}(v) \phi_{i}(y)+\lambda_{v}\right] \eta d x \\
& +\left.2 \beta_{\mathrm{v}^{\prime} i}^{0} \frac{d v}{d x} \eta\right|_{L} ^{0}+\left.\sum_{i} \beta_{\mathrm{v}^{\prime} i} \phi_{i}(v) 2 \frac{d v}{d x} \eta\right|_{L} ^{0}
\end{aligned}
$$

From Equation 6.7, we obtain the corresponding Euler-Lagrange equation as below:

$$
\begin{aligned}
& 2 n_{\mathrm{os}} F \varphi+n_{\mathrm{os}} R T \log \frac{v}{1-v}+\sum_{i} \beta_{\mathrm{v} i} \phi_{i}^{\prime}(v)-2 \beta_{\mathrm{v}^{\prime} i}^{0} \frac{d^{2} v}{d x^{2}}+\sum_{i} \beta_{\mathrm{v}^{\prime} i} \phi_{i}^{\prime}(v)\left(\frac{d v}{d x}\right)^{2} \\
& -\sum_{i} \beta_{\mathrm{v}^{\prime} i} \phi_{i}^{\prime}(v) 2 \frac{d v}{d x}-\sum_{i} \beta_{\mathrm{v}^{\prime} i} \phi_{i}(v) 2 \frac{d^{2} v}{d x^{2}}+\sum_{i} \beta_{\mathrm{vy}^{2}} \phi_{i}^{\prime}(v) \phi_{i}(y)+\lambda_{v}=0
\end{aligned}
$$

Accordingly, the boundary condition that naturally arises is:

$$
\left.2 \beta_{\mathrm{v}^{\prime} i}^{0} \frac{d v}{d x}\right|_{x=0}+\left.\sum_{i} \beta_{\mathrm{v}^{\prime} i} \phi_{i}(v) 2 \frac{d v}{d x}\right|_{x=0}=n_{\mathrm{o}} f_{\mathrm{o}}
$$

Multiplying Equation 6.8 by the weight function w and integrating over the whole 
domain gives:

$$
\begin{aligned}
& \int_{0}^{L} 2 n_{\mathrm{os}} F \varphi \mathrm{w} d x+\int_{0}^{L} n_{\mathrm{os}} R T \log \frac{v}{1-v} \mathrm{w} d x \\
& +\int_{0}^{L}\left[\sum_{i} \beta_{\mathrm{v} i} \phi_{i}^{\prime}(v)-2 \beta_{\mathrm{v}^{\prime} i}^{0} \frac{d^{2} v}{d x^{2}}+\sum_{i} \beta_{\mathrm{v}^{\prime} i} \phi_{i}^{\prime}(v)\left(\frac{d v}{d x}\right)^{2}\right. \\
& \left.-\sum_{i} \beta_{\mathrm{v}^{\prime} i} \phi_{i}^{\prime}(v) 2 \frac{d v}{d x}-\sum_{i} \beta_{\mathrm{v}^{\prime} i} \phi_{i}(v) 2 \frac{d^{2} v}{d x^{2}}+\sum_{i} \beta_{\mathrm{vy} i} \phi_{i}^{\prime}(v) \phi_{i}(y)+\lambda_{v}\right] \mathrm{w} d x=0
\end{aligned}
$$

The obtained equation can be further simplified because:

$$
\begin{aligned}
& \int_{0}^{L}\left[-2 \beta_{\mathrm{v}^{\prime} i}^{0} \frac{d^{2} v}{d x^{2}}-\sum_{i} \beta_{\mathrm{v}^{\prime} i} \phi_{i}^{\prime}(v) 2 \frac{d v}{d x}-\sum_{i} \beta_{\mathrm{v}^{\prime} i} \phi_{i}(v) 2 \frac{d^{2} v}{d x^{2}}\right] \mathrm{w} d x \\
& =\int_{0}^{L} d\left[-2 \beta_{\mathrm{v}^{\prime} i}^{0} \frac{d v}{d x}-2 \sum_{i} \beta_{\mathrm{v}^{\prime} i} \phi_{i}(v) \frac{d v}{d x}\right] \mathrm{w} d x=-2 \beta_{\mathrm{v}^{\prime} i}^{0} \frac{d v}{d x} \mathrm{w}_{L}^{0}-\left.2 \sum_{i} \beta_{\mathrm{v}^{\prime} i} \phi_{i}(v) \frac{d v}{d x} \mathrm{w}\right|_{L} ^{0} \\
& +\int_{0}^{L}\left[2 \beta_{\mathrm{v}^{\prime} i}^{0} \frac{d v}{d x}+2 \sum_{i} \beta_{\mathrm{v}^{\prime} i} \phi_{i}(v) \frac{d v}{d x}\right] d \mathrm{w} d x
\end{aligned}
$$

This yields the boundary condition below:

$$
\left.\left[2 \beta_{\mathrm{v}^{\prime} i}^{0} \frac{d v}{d x}+\sum_{i} \beta_{\mathrm{v}^{\prime} i} \phi_{i}(v) 2 \frac{d v}{d x}\right] \mathrm{w}\right|_{x=0}=0
$$

Therefore, Equation 6.10 becomes:

$$
\begin{aligned}
& \int_{0}^{L} 2 n_{\mathrm{os}} F \varphi \mathrm{w} d x+\int_{0}^{L} n_{\mathrm{os}} R T \log \frac{v}{1-v} \mathrm{w} d x+\int_{0}^{L}\left[\sum_{i} \beta_{\mathrm{v} i} \phi_{i}^{\prime}(v)+\sum_{i} \beta_{\mathrm{v}^{\prime} i} \phi_{i}^{\prime}(v)\left(\frac{d v}{d x}\right)^{2}\right. \\
& \left.+\sum_{i} \beta_{\mathrm{vy} i} \phi_{i}^{\prime}(v) \phi_{i}(y)+\lambda_{v}\right] \mathrm{w} d x+\int_{0}^{L}\left[2 \beta_{\mathrm{v}^{\prime} i}^{0} \frac{d v}{d x}+2 \sum_{i} \beta_{\mathrm{v}^{\prime} i} \phi_{i}(v) \frac{d v}{d x}\right] d \mathrm{w} d x=0
\end{aligned}
$$


Using linear basis functions for $\mathrm{w}$, the discretized representation for node $\mathrm{j}$ becomes:

$$
\begin{aligned}
& \int_{x_{j-1}}^{x_{j}} 2 n_{\mathrm{os}} F \varphi_{j} \mathrm{w}_{j} d x+\int_{x_{j}}^{x_{j+1}} 2 n_{\mathrm{os}} F \varphi_{j} \mathrm{w}_{j} d x+\int_{x_{j-1}}^{x_{j}} n_{\mathrm{os}} R T \log \frac{v_{j}}{1-v_{j}} \mathrm{w}_{j} d x \\
& +\int_{x_{j}}^{x_{j+1}} n_{\mathrm{os}} R T \log \frac{v_{j}}{1-v_{j}} \mathrm{w}_{j} d x+\int_{x_{j-1}}^{x_{j}} \sum_{i} \beta_{\mathrm{vi}} \phi_{i}^{\prime}\left(v_{j}\right) \mathrm{w}_{j} d x+\int_{x_{j}}^{x_{j+1}} \sum_{i} \beta_{\mathrm{vi}} \phi_{i}^{\prime}\left(v_{j}\right) \mathrm{w}_{j} d x \\
& +\int_{x_{j-1}}^{x_{j}} \sum_{i} \beta_{\mathrm{v}^{\prime} i} \phi_{i}^{\prime}\left(v_{j}\right)\left(\frac{v_{j}-v_{j-1}}{h_{j-1}}\right)^{2} \mathrm{w}_{j} d x+\int_{x_{j}}^{x_{j+1}} \sum_{i} \beta_{\mathrm{v}^{\prime} i} \phi_{i}^{\prime}\left(v_{j}\right)\left(\frac{v_{j+1}-v_{j}}{h_{j}}\right)^{2} \mathrm{w}_{j} d x \\
& +\int_{x_{j-1}}^{x_{j}} \sum_{i} \beta_{\mathrm{vyy}_{i}} \phi_{i}^{\prime}\left(v_{j}\right) \phi_{i}\left(y_{j}\right) \mathrm{w}_{j} d x+\int_{x_{j}}^{x_{j+1}} \sum_{i} \beta_{\mathrm{vy} i} \phi_{i}^{\prime}\left(v_{j}\right) \phi_{i}\left(y_{j}\right) \mathrm{w}_{j} d x+\int_{x_{j-1}}^{x_{j+1}} \lambda_{v} d x \\
& +\int_{x_{j-1}}^{x_{j}} 2 \beta_{\mathrm{v}^{\prime} i}^{0} \frac{v_{j}-v_{j-1}}{h_{j-1}} \cdot \frac{1}{h_{j-1}} d x+\int_{x_{j}}^{x_{j+1}} 2 \beta_{\mathrm{v}^{\prime} i}^{0} \frac{v_{j+1}-v_{j}}{h_{j}} \cdot \frac{-1}{h_{j}} d x \\
& +\int_{x_{j-1}}^{x_{j}} 2 \sum_{i} \beta_{\mathrm{v}^{\prime} i} \phi_{i}\left(v_{j}\right) \frac{v_{j}-v_{j-1}}{h_{j-1}} \cdot \frac{1}{h_{j-1}} d x+\int_{x_{j}}^{x_{j+1}} 2 \sum_{i} \beta_{\mathrm{v}^{\prime} i} \phi_{i}\left(v_{j}\right) \frac{v_{j+1}-v_{j}}{h_{j}} \cdot \frac{-1}{h_{j}} d x=0
\end{aligned}
$$

Utilizing the representation for the linear basis functions and applying the trapezoidal rule, Equation 6.14 becomes

$$
\begin{aligned}
& \frac{h_{j-1}+h_{j}}{2} 2 n_{\mathrm{os}} F \varphi_{j}+\frac{h_{j-1}+h_{j}}{2} n_{\mathrm{os}} R T \log \frac{v_{j}}{1-v_{j}} \\
& +\frac{h_{j-1}+h_{j}}{2} \sum_{i} \beta_{\mathrm{v} i} \phi_{i}^{\prime}\left(v_{j}\right)+\frac{h_{j-1}}{2} \sum_{i} \beta_{\mathrm{v}^{\prime} i} \phi_{i}^{\prime}\left(v_{j}\right)\left(\frac{v_{j}-v_{j-1}}{h_{j-1}}\right)^{2} \\
& +\frac{h_{j}}{2} \sum_{i} \beta_{\mathrm{v}^{\prime} i} \phi_{i}^{\prime}\left(v_{j}\right)\left(\frac{v_{j+1}-v_{j}}{h_{j}}\right)^{2}+\frac{h_{j-1}+h_{j}}{2} \sum_{i} \beta_{\mathrm{vy} i} \phi_{i}^{\prime}\left(v_{j}\right) \phi_{i}\left(y_{j}\right)+\frac{h_{j-1}+h_{j}}{2} \lambda_{v} \\
& +\left[2 \beta_{\mathrm{v}^{\prime} i}^{0}+\sum_{i} \beta_{\mathrm{v}^{\prime} i} \phi_{i}\left(v_{j}\right)\right] \frac{v_{j}-v_{j-1}}{h_{j-1}}-\left[2 \beta_{\mathrm{v}^{\prime} i}^{0}+\sum_{i} \beta_{\mathrm{v}^{\prime} i} \phi_{i}\left(v_{j}\right)\right] \frac{v_{j+1}-v_{j}}{h_{j}}=0
\end{aligned}
$$

Stencil equations for the dopant $y$ can be derived in a similar fashion.

Take variational differentiation of the free energy functional 6.3 with respect to y. Let $y=f+\varepsilon \eta$, where $\eta(x)$ is an arbitrary function that is at least first-order differentiable and becomes zero at both endpoints of the domain. $f$ is where the functional attains a minimum. Thus, the functional is minimized when $\varepsilon=0$. Taking the total derivative of $\Omega\left(y,\left(\frac{d y}{d x}\right)^{2}\right)$, 
where $y=f+\varepsilon \eta$ and $y^{\prime}=f^{\prime}+\varepsilon \eta^{\prime}$ are functions of $\varepsilon$ but $\mathrm{x}$ is not,

$$
\begin{aligned}
\frac{d \Omega}{d \varepsilon}= & \frac{\partial \Omega_{0}}{\partial y} \frac{d y}{d \varepsilon}+\frac{\partial \Omega_{0}}{\partial y^{\prime}} \frac{d y}{d \varepsilon}+\int_{0}^{L}\left[\sum_{i} \beta_{\mathrm{v}_{i}} \phi_{i}^{\prime}(v) \eta+2 \beta_{\mathrm{v}^{\prime} i}^{0} \frac{d v}{d x} \eta^{\prime}+\sum_{i} \beta_{\mathrm{v}^{\prime} i} \phi_{i}^{\prime}(v)\left(\frac{d v}{d x}\right)^{2} \eta\right. \\
& \left.+\sum_{i} \beta_{\mathrm{v}^{\prime} i} \phi_{i}(v) 2 \frac{d v}{d x} \eta^{\prime}+\sum_{i} \beta_{\mathrm{vy} i} \phi_{i}^{\prime}(v) \phi_{i}(y) \eta+\lambda_{v} \eta\right] d x
\end{aligned}
$$

Applying integration by parts to the terms containing $\eta^{\prime}$ in the above equation and reorganizing terms related to $\eta$ yields:

$$
\begin{aligned}
\frac{d \Omega}{d \varepsilon}= & \frac{\partial \Omega_{0}}{\partial y} \frac{d y}{d \varepsilon}+\frac{\partial \Omega_{0}}{\partial y^{\prime}} \frac{d y}{d \varepsilon}+\int_{0}^{L}\left[\sum_{i} \beta_{\mathrm{y} i} \phi_{i}^{\prime}(y)+\sum_{i} \beta_{\mathrm{y}^{\prime} i} \phi_{i}^{\prime}(y)\left(\frac{d y}{d x}\right)^{2}\right. \\
& \left.-2 \beta_{\mathrm{y}^{\prime} i}^{0} \frac{d^{2} y}{d x^{2}}-\sum_{i} \beta_{\mathrm{y}^{\prime} i} \phi_{i}^{\prime}(y) 2 \frac{d y}{d x}-\sum_{i} \beta_{\mathrm{y}^{\prime} i} \phi_{i}(y) 2 \frac{d^{2} y}{d x^{2}}+\sum_{i} \beta_{\mathrm{vy} i} \phi_{i}^{\prime}(y) \phi_{i}(v)+\lambda_{y}\right] \eta d x \\
& +\left.2 \beta_{\mathrm{y}^{\prime} i}^{0} \frac{d y}{d x} \eta\right|_{L} ^{0}+\left.\sum_{i} \beta_{\mathrm{y}^{\prime} i} \phi_{i}(y) 2 \frac{d y}{d x} \eta\right|_{L} ^{0}
\end{aligned}
$$

From Equation 6.17, we obtain the corresponding Euler-Lagrange equation as below:

$$
\begin{aligned}
& -n_{\mathrm{cs}} F \varphi+n_{\mathrm{cs}} R T \log \frac{y}{1-y}+\sum_{i} \beta_{\mathrm{y}_{i}} \phi_{i}^{\prime}(y)-2 \beta_{\mathrm{y}^{\prime} i}^{0} \frac{d^{2} y}{d x^{2}}+\sum_{i} \beta_{\mathrm{y}^{\prime} i} \phi_{i}^{\prime}(y)\left(\frac{d y}{d x}\right)^{2} \\
& -\sum_{i} \beta_{\mathrm{y}^{\prime} i} \phi_{i}^{\prime}(y) 2 \frac{d y}{d x}-\sum_{i} \beta_{\mathrm{y}^{\prime} i} \phi_{i}(y) 2 \frac{d^{2} y}{d x^{2}}+\sum_{i} \beta_{\mathrm{vy} i} \phi_{i}^{\prime}(y) \phi_{i}(v)+\lambda_{y}=0
\end{aligned}
$$

Accordingly, the boundary condition that naturally arises is:

$$
\left.2 \beta_{\mathrm{y}^{\prime} i}^{0} \frac{d y}{d x}\right|_{x=0}+\left.\sum_{i} \beta_{\mathrm{y}^{\prime} i} \phi_{i}(y) 2 \frac{d y}{d x}\right|_{x=0}=0
$$

Multiplying Equation 6.18 by the weight function w and integrating over the whole 
domain gives:

$$
\begin{aligned}
& \int_{0}^{L}-n_{\mathrm{cs}} F \varphi \mathrm{w} d x+\int_{0}^{L} n_{\mathrm{cs}} R T \log \frac{y}{1-y} \mathrm{w} d x \\
& +\int_{0}^{L}\left[\sum_{i} \beta_{\mathrm{y} i} \phi_{i}^{\prime}(y)-2 \beta_{\mathrm{y}^{\prime} i}^{0} \frac{d^{2} y}{d x^{2}}+\sum_{i} \beta_{\mathrm{y}^{\prime} i} \phi_{i}^{\prime}(y)\left(\frac{d y}{d x}\right)^{2}\right. \\
& \left.-\sum_{i} \beta_{\mathrm{y}^{\prime} i} \phi_{i}^{\prime}(y) 2 \frac{d y}{d x}-\sum_{i} \beta_{\mathrm{y}^{\prime} i} \phi_{i}(y) 2 \frac{d^{2} y}{d x^{2}}+\sum_{i} \beta_{\mathrm{vy} i} \phi_{i}^{\prime}(y) \phi_{i}(v)+\lambda_{y}\right] \mathrm{w} d x=0
\end{aligned}
$$

The obtained equation can be further simplified because:

$$
\begin{aligned}
& \int_{0}^{L}\left[-2 \beta_{\mathrm{y}^{\prime} i}^{0} \frac{d^{2} y}{d x^{2}}-\sum_{i} \beta_{\mathrm{y}^{\prime} i} \phi_{i}^{\prime}(y) 2 \frac{d y}{d x}-\sum_{i} \beta_{\mathrm{y}^{\prime} i} \phi_{i}(y) 2 \frac{d^{2} y}{d x^{2}}\right] \mathrm{w} d x \\
& =\int_{0}^{L} d\left[-2 \beta_{\mathrm{y}^{\prime} i}^{0} \frac{d y}{d x}-2 \sum_{i} \beta_{\mathrm{y}^{\prime} i} \phi_{i}(y) \frac{d y}{d x}\right] \mathrm{w} d x=-\left.2 \beta_{\mathrm{y}^{\prime} i}^{0} \frac{d y}{d x} \mathrm{w}\right|_{L} ^{0}-\left.2 \sum_{i} \beta_{\mathrm{v}^{\prime} i} \phi_{i}(v) \frac{d v}{d x} \mathrm{w}\right|_{L} ^{0} \\
& +\int_{0}^{L}\left[2 \beta_{\mathrm{y}^{\prime} i}^{0} \frac{d y}{d x}+2 \sum_{i} \beta_{\mathrm{y}^{\prime} i} \phi_{i}(y) \frac{d y}{d x}\right] d \mathrm{w} d x
\end{aligned}
$$

This yields the boundary condition below:

$$
\left.\left[2 \beta_{\mathrm{y}^{\prime} i}^{0} \frac{d y}{d x}+\sum_{i} \beta_{\mathrm{y}^{\prime} i} \phi_{i}(y) 2 \frac{d y}{d x}\right] \mathrm{w}\right|_{x=0}=0
$$

Therefore, Equation 6.20 becomes:

$$
\begin{aligned}
& \int_{0}^{L}-n_{\mathrm{cs}} F \varphi \mathrm{w} d x+\int_{0}^{L} n_{\mathrm{cs}} R T \log \frac{y}{1-y} \mathrm{w} d x+\int_{0}^{L}\left[\sum_{i} \beta_{\mathrm{y} i} \phi_{i}^{\prime}(y)+\sum_{i} \beta_{\mathrm{y}^{\prime} i} \phi_{i}^{\prime}(y)\left(\frac{d y}{d x}\right)^{2}\right. \\
& \left.+\sum_{i} \beta_{\mathrm{vy} i} \phi_{i}^{\prime}(y) \phi_{i}(v)+\lambda_{y}\right] \mathrm{w} d x+\int_{0}^{L}\left[2 \beta_{\mathrm{y}^{\prime} i}^{0} \frac{d y}{d x}+2 \sum_{i} \beta_{\mathrm{y}^{\prime} i} \phi_{i}(y) \frac{d y}{d x}\right] d \mathrm{w} d x=0
\end{aligned}
$$


Using linear basis functions for $\mathrm{w}$, the discretized representation for node $\mathrm{j}$ becomes:

$$
\begin{aligned}
& \int_{x_{j-1}}^{x_{j}}-n_{\mathrm{cs}} F \varphi_{j} \mathrm{w}_{j} d x-\int_{x_{j}}^{x_{j+1}} n_{\mathrm{cs}} F \varphi_{j} \mathrm{w}_{j} d x+\int_{x_{j-1}}^{x_{j}} n_{\mathrm{cs}} R T \log \frac{y_{j}}{1-y_{j}} \mathrm{w}_{j} d x \\
& +\int_{x_{j}}^{x_{j+1}} n_{\mathrm{cs}} R T \log \frac{y_{j}}{1-y_{j}} \mathrm{w}_{j} d x+\int_{x_{j-1}}^{x_{j}} \sum_{i} \beta_{\mathrm{y} i} \phi_{i}^{\prime}\left(y_{j}\right) \mathrm{w}_{j} d x+\int_{x_{j}}^{x_{j+1}} \sum_{i} \beta_{\mathrm{y} i} \phi_{i}^{\prime}\left(y_{j}\right) \mathrm{w}_{j} d x \\
& +\int_{x_{j-1}}^{x_{j}} \sum_{i} \beta_{\mathrm{y}^{\prime} i} \phi_{i}^{\prime}\left(y_{j}\right)\left(\frac{y_{j}-y_{j-1}}{h_{j-1}}\right)^{2} \mathrm{w}_{j} d x+\int_{x_{j}}^{x_{j+1}} \sum_{i} \beta_{\mathrm{y}^{\prime} i} \phi_{i}^{\prime}\left(y_{j}\right)\left(\frac{y_{j+1}-y_{j}}{h_{j}}\right)^{2} \mathrm{w}_{j} d x \\
& +\int_{x_{j-1}}^{x_{j}} \sum_{i} \beta_{\mathrm{vy} i} \phi_{i}^{\prime}\left(y_{j}\right) \phi_{i}\left(v_{j}\right) \mathrm{w}_{j} d x+\int_{x_{j}}^{x_{j+1}} \sum_{i} \beta_{\mathrm{vy}_{i}} \phi_{i}^{\prime}\left(y_{j}\right) \phi_{i}\left(v_{j}\right) \mathrm{w}_{j} d x+\int_{x_{j-1}}^{x_{j+1}} \lambda_{y} d x \\
& +\int_{x_{j-1}}^{x_{j}} 2 \beta_{\mathrm{y}^{\prime} i}^{0} \frac{y_{j}-y_{j-1}}{h_{j-1}} \cdot \frac{1}{h_{j-1}} d x+\int_{x_{j}}^{x_{j+1}} 2 \beta_{\mathrm{y}^{\prime} i}^{0} \frac{y_{j+1}-y_{j}}{h_{j}} \cdot \frac{-1}{h_{j}} d x \\
& +\int_{x_{j-1}}^{x_{j}} 2 \sum_{i} \beta_{\mathrm{y}^{\prime} i} \phi_{i}\left(y_{j}\right) \frac{y_{j}-y_{j-1}}{h_{j-1}} \cdot \frac{1}{h_{j-1}} d x+\int_{x_{j}}^{x_{j+1}} 2 \sum_{i} \beta_{\mathrm{y}^{\prime} i} \phi_{i}\left(y_{j}\right) \frac{y_{j+1}-y_{j}}{h_{j}} \cdot \frac{-1}{h_{j}} d x=0
\end{aligned}
$$

Utilizing the representation for the linear basis functions and applying the trapezoidal rule, Equation 6.24 becomes

$$
\begin{aligned}
& -\frac{h_{j-1}+h_{j}}{2} n_{\mathrm{cs}} F \varphi_{j}+\frac{h_{j-1}+h_{j}}{2} n_{\mathrm{cs}} R T \log \frac{y_{j}}{1-y_{j}} \\
& +\frac{h_{j-1}+h_{j}}{2} \sum_{i} \beta_{\mathrm{yi}} \phi_{i}^{\prime}\left(y_{j}\right)+\frac{h_{j-1}}{2} \sum_{i} \beta_{\mathrm{y}^{\prime} i} \phi_{i}^{\prime}\left(y_{j}\right)\left(\frac{y_{j}-y_{j-1}}{h_{j-1}}\right)^{2} \\
& +\frac{h_{j}}{2} \sum_{i} \beta_{\mathrm{y}^{\prime} i} \phi_{i}^{\prime}\left(y_{j}\right)\left(\frac{y_{j+1}-y_{j}}{h_{j}}\right)^{2}+\frac{h_{j-1}+h_{j}}{2} \sum_{i} \beta_{\mathrm{vy} i} \phi_{i}^{\prime}\left(y_{j}\right) \phi_{i}\left(v_{j}\right)+\frac{h_{j-1}+h_{j}}{2} \lambda_{y} \\
& +\left[2 \beta_{\mathrm{y}^{\prime} i}^{0}+\sum_{i} \beta_{\mathrm{y}^{\prime} i} \phi_{i}\left(y_{j}\right)\right] \frac{y_{j}-y_{j-1}}{h_{j-1}}-\left[2 \beta_{\mathrm{y}^{\prime} i}^{0}+\sum_{i} \beta_{\mathrm{y}^{\prime} i} \phi_{i}\left(y_{j}\right)\right] \frac{y_{j+1}-y_{j}}{h_{j}}=0
\end{aligned}
$$

Variational analysis with respect to $\varphi$ leads to the Euler-Lagrange equation below:

$$
\begin{aligned}
\frac{d \Omega}{d \varepsilon}= & \frac{\partial \Omega}{\partial \varphi} \frac{d \varphi}{d \varepsilon}+\frac{\partial \Omega}{\partial \varphi^{\prime}} \frac{d \varphi^{\prime}}{d \varepsilon} \\
& =\int_{0}^{L}\left[-\varepsilon_{r} \varepsilon_{0}\left(\frac{d \varphi}{d x}\right) \eta^{\prime}+F\left(2 n_{o s} v-n_{c s} y\right) \eta\right] d x
\end{aligned}
$$

Applying integration by parts to the terms containing $\eta^{\prime}$ in the above equation and reorga- 
nizing terms related to $\eta$ yields:

$$
\frac{d \Omega}{d \varepsilon}=\int_{0}^{L}\left[\varepsilon_{r} \varepsilon_{0}\left(\frac{d^{2} \varphi}{d x^{2}}\right) \eta+F\left(2 n_{o s} v-n_{c s} y\right) \eta\right] d x-\left.\varepsilon_{r} \varepsilon_{0} \frac{d \varphi}{d x} \eta\right|_{0} ^{L}
$$

The boundary condition arising naturally from the variational analysis is:

$$
\left.\frac{d \varphi}{d x}\right|_{0}=0
$$

Here we obtain the Euler-Lagrange equation for $\varphi$ as below:

$$
\varepsilon_{r} \varepsilon_{0}\left(\frac{d^{2} \varphi}{d x^{2}}\right)+F\left(2 n_{o s} v-n_{c s} y\right)=0
$$

Multiplying Equation 6.8 by the weight function $\mathrm{w}$ and integrating over the whole domain gives:

$$
\int_{0}^{L}\left[\varepsilon_{r} \varepsilon_{0}\left(\frac{d^{2} \varphi}{d x^{2}}\right)+F\left(2 n_{o s} v-n_{c s} y\right)\right] \mathrm{w} d x=0
$$

The first term of the above equation can be further represented as:

$$
\int_{0}^{L} \varepsilon_{r} \varepsilon_{0}\left(\frac{d^{2} \varphi}{d x^{2}}\right) \mathrm{w} d x=\left.\varepsilon_{r} \varepsilon_{0}\left(\frac{d \varphi}{d x}\right) \mathrm{w}\right|_{0} ^{L}-\int_{0}^{L} \varepsilon_{r} \varepsilon_{0}\left(\frac{d \varphi}{d x}\right) d \mathrm{w} d x=-\int_{0}^{L} \varepsilon_{r} \varepsilon_{0}\left(\frac{d \varphi}{d x}\right) d \mathrm{w} d x
$$

Therefore Equation 6.30 can be simplified as:

$$
\int_{0}^{L}\left[F\left(2 n_{o s} v-n_{c s} y\right) \mathrm{w}-\varepsilon_{r} \varepsilon_{0}\left(\frac{d \varphi}{d x}\right) d \mathrm{w}\right] d x=0
$$


Using linear basis functions for $\mathrm{w}$, the discretized representation for node $\mathrm{j}$ becomes:

$$
\begin{aligned}
& \int_{x_{j-1}}^{x_{j}} F\left(2 n_{o s} v_{j}-n_{c s} y_{j}\right) \mathrm{w}_{j} d x+\int_{x_{j}}^{x_{j+1}} F\left(2 n_{o s} v_{j}-n_{c s} y_{j}\right) \mathrm{w}_{j} d x \\
& -\int_{x_{j-1}}^{x_{j}} \varepsilon_{r} \varepsilon_{0} \frac{\varphi_{j}-\varphi_{j-1}}{h_{j-1}} \cdot \frac{1}{h_{j-1}} d x-\int_{x_{j}}^{x_{j+1}} \varepsilon_{r} \varepsilon_{0} \frac{\varphi_{j+1}-\varphi_{j}}{h_{j}} \cdot \frac{-1}{h_{j}} d x=0
\end{aligned}
$$

Utilizing the representation for the linear basis functions and applying the trapezoidal rule, Equation 6.33 becomes

$$
\frac{h_{j-1}+h_{j}}{2} F\left(2 n_{o s} v_{j}-n_{c s} y_{j}\right)+\varepsilon_{r} \varepsilon_{0} \frac{\varphi_{j-1}-\varphi_{j}}{h_{j-1}}+\varepsilon_{r} \varepsilon_{0} \frac{\varphi_{j+1}-\varphi_{j}}{h_{j}}=0
$$

\subsection{Systematic Model Building with Bayesian Calibration}

A series of models are formulated following the general model derivation in 6.1. The baseline model is representative of the traditional Gouy-Chapman model, where no defect interaction or gradient energy terms are considered. With later model versions, defect interactions and gradient energy terms are systematically built into the model structure. The table below shows the effects included in each model version explicitly. 


\begin{tabular}{|l|l|l|l|l|l|l|}
\hline Param & $n_{o}$ & $f_{y}$ & $f_{y v}$ & $f_{v}$ & $c_{y}$ & $c_{v}$ \\
\hline V0 & $\mathrm{X}$ & & & & & \\
V1 & $\mathrm{X}$ & $\mathrm{X}$ & & & & \\
V3 & $\mathrm{X}$ & $\mathrm{X}$ & & & & \\
V4 & $\mathrm{X}$ & $\mathrm{X}$ & & $\mathrm{X}$ & & \\
V5 & $\mathrm{X}$ & & $\mathrm{X}$ & $\mathrm{X}$ & & \\
V6 & $\mathrm{X}$ & $\mathrm{X}$ & $\mathrm{X}$ & & & \\
V7 & $\mathrm{X}$ & $\mathrm{X}$ & & & $\mathrm{X}$ & \\
V8 & $\mathrm{X}$ & & & $\mathrm{X}$ & & $\mathrm{X}$ \\
V9 & $\mathrm{X}$ & $\mathrm{X}$ & $\mathrm{X}$ & $\mathrm{X}$ & & \\
V10 & $\mathrm{X}$ & $\mathrm{X}$ & & $\mathrm{X}$ & $\mathrm{X}$ & \\
V11 & $\mathrm{X}$ & $\mathrm{X}$ & & $\mathrm{X}$ & & $\mathrm{X}$ \\
V12 & $\mathrm{X}$ & $\mathrm{X}$ & $\mathrm{X}$ & & $\mathrm{X}$ & \\
V13 & $\mathrm{X}$ & & $\mathrm{X}$ & $\mathrm{X}$ & & $\mathrm{X}$ \\
V14 & $\mathrm{X}$ & $\mathrm{X}$ & & $\mathrm{X}$ & $\mathrm{X}$ & $\mathrm{X}$ \\
V15 & $\mathrm{X}$ & $\mathrm{X}$ & $\mathrm{X}$ & $\mathrm{X}$ & $\mathrm{X}$ & \\
V16 & $\mathrm{X}$ & $\mathrm{X}$ & $\mathrm{X}$ & $\mathrm{X}$ & & $\mathrm{X}$ \\
V17 & $\mathrm{X}$ & $\mathrm{X}$ & $\mathrm{X}$ & $\mathrm{X}$ & $\mathrm{X}$ & $\mathrm{X}$ \\
V18 & $\mathrm{X}$ & $\mathrm{XX}$ & $\mathrm{X}$ & & $\mathrm{X}$ & \\
V19 & $\mathrm{X}$ & $\mathrm{XX}$ & $\mathrm{X}$ & $\mathrm{XX}$ & $\mathrm{X}$ & $\mathrm{X}$ \\
V20 & $\mathrm{X}$ & $\mathrm{XX}$ & $\mathrm{X}$ & $\mathrm{XX}$ & $\mathrm{XX}$ & $\mathrm{XX}$ \\
$\mathrm{V} 21$ & $\mathrm{X}$ & $\mathrm{XX}$ & $\mathrm{XX}$ & $\mathrm{XX}$ & $\mathrm{XX}$ & $\mathrm{XX}$ \\
\hline
\end{tabular}

Table 6.1: Design of Model structures through Systematic Building

In Table 6.1, for each model version, $\mathrm{X}$ in the corresponding parameter field means 
that one basis function of the corresponding effect is incorporated into the model, and XX means that more than one basis function are incorporated.

Applicability of any model has to be validated with experimental data. In this study, Ca profiles near the GB of $\mathrm{CaxCe}_{1-\mathrm{x}} \mathrm{O}_{2-\mathrm{x}}$ measured using electron energy-loss spectroscopy (EELS) for three different concentrations corresponding to $x=0.02,0.05,0.1$ [2] are all utilized for model building and validation. In later context, the profiles pertain to three different concentrations are denoted CCO2, CCO5 and CCO10, respectively. Figure 6.1, 6.2 and 6.3 display dopant concentrations near grain boundaries of different angels for CCO2, CCO5 and CCO10. 


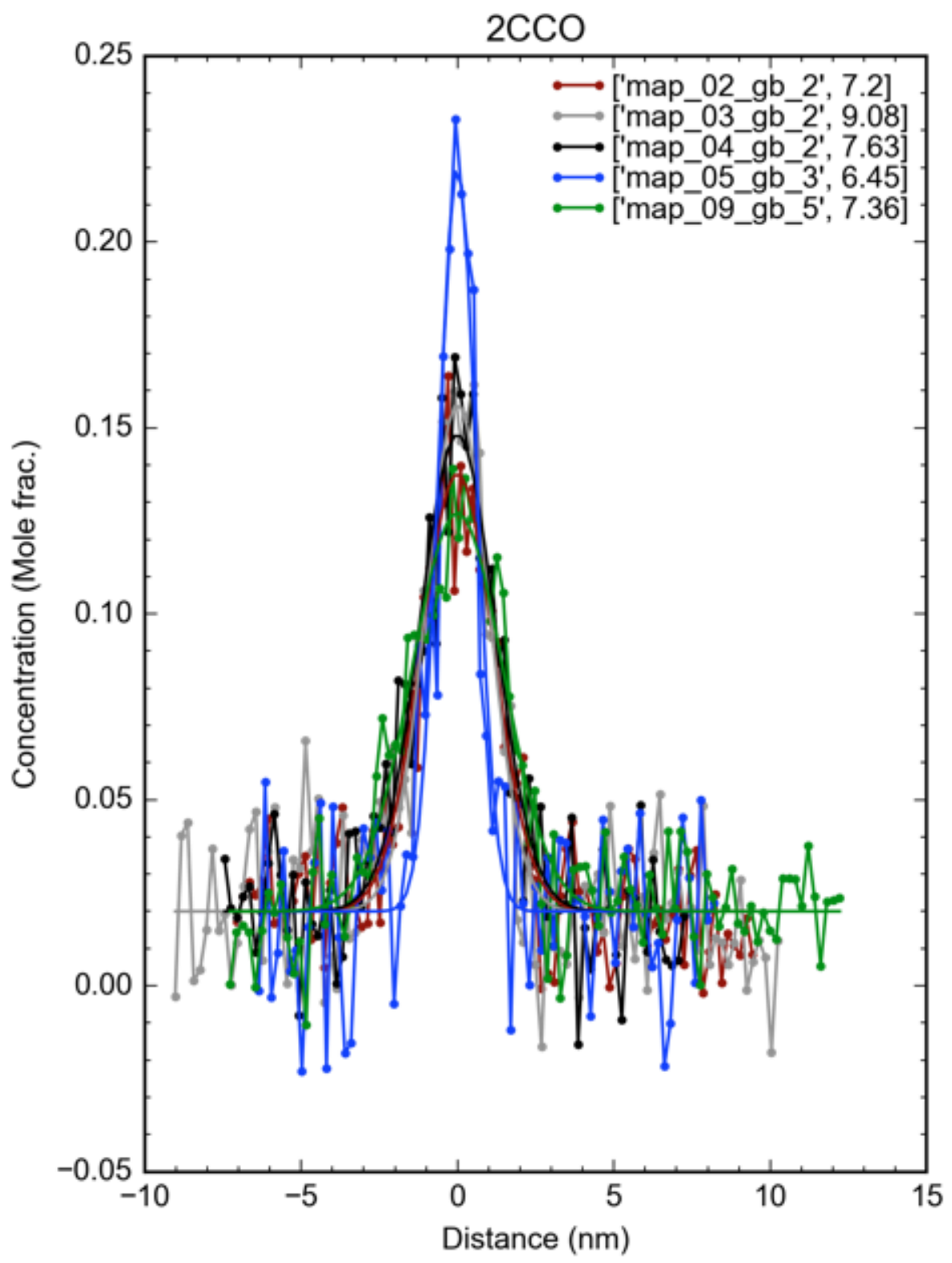

Figure 6.1: Experimental data of CCO2 [2] 


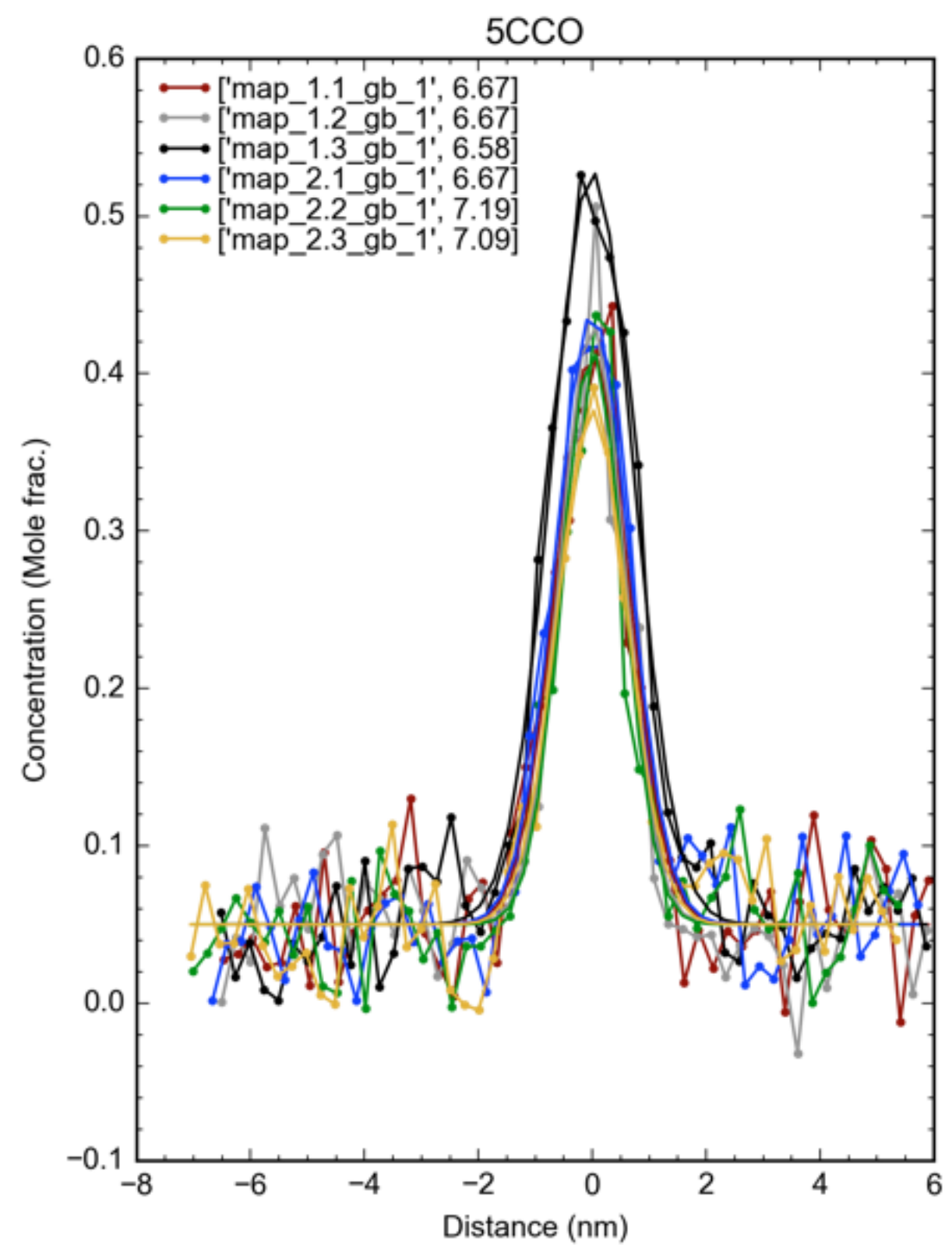

Figure 6.2: Experimental data of CCO5 [2] 


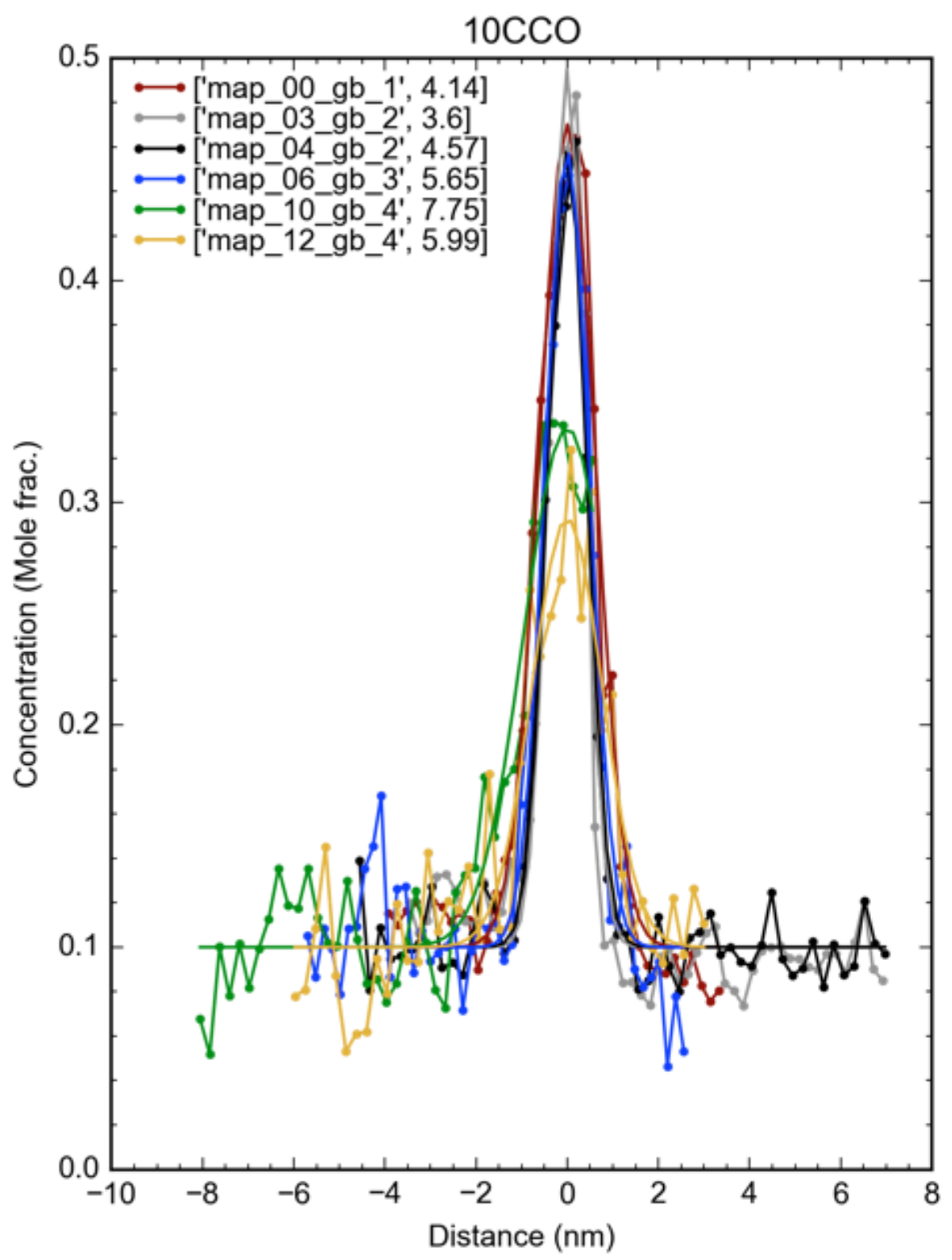

Figure 6.3: Experimental data of CCO10 [2]

For computational use, profiles around the two sides of the GB are treated as two separate datasets and potential outliers are removed. With these experimental measurement of Ca profiles near GBs, bayesian calibration is employed to each model in order to identify parameter posterior distributions. This process enables identification of the optimal model parameter space and model structure using machine learning method guided by experimental data. Results from the systematic model building process naturally reveal the significance 
of different factors in modeling the grain boundary effect.

Bayesian calibration is performed for all model version from V0 to V20 using the same MCMC routine with block proposal as in Section 5.2. For each calibration, the same model structure and parameter values are evaluated for bulk Ca concentrations of 2\%, $5 \%$ and $10 \%$, respectively. This enables the realization of a unified model for solid solutions of different concentrations. The MCMC process was cutoff at different steps for each model calibration depending on when the burning-in took place and when enough posterior samples were obtained. The amount of model evaluation time also varies for different model version. This was also taken into consideration when deciding the total number of MCMC steps to be executed. The total number of MCMC steps and burning-in cutoff steps are summaries in Table 6.2 for each calibration. 


\begin{tabular}{|c|c|c|}
\hline Model Version & Total MCMC Steps & Burning-in Cutoff \\
\hline V0 & 23701 & 2000 \\
\hline V1 & 21301 & 3000 \\
\hline $\mathrm{V} 2$ & 31501 & 3000 \\
\hline V3 & 50101 & 3000 \\
\hline $\mathrm{V} 4$ & 30901 & 3000 \\
\hline V5 & 26101 & 3000 \\
\hline V6 & 15301 & 2000 \\
\hline V7 & 43801 & 2000 \\
\hline V8 & 45901 & 3000 \\
\hline V9 & 82501 & 10000 \\
\hline V10 & 13501 & 2000 \\
\hline V11 & 50101 & 2000 \\
\hline V12 & 11101 & 2000 \\
\hline V13 & 71401 & 2000 \\
\hline V14 & 28501 & 2000 \\
\hline V15 & 30901 & 2000 \\
\hline V16 & 7861 & 2000 \\
\hline V17 & 10801 & 4000 \\
\hline V18 & 21001 & 2000 \\
\hline V19 & 23101 & 2000 \\
\hline V20 & 81601 & 2000 \\
\hline V21 & 25501 & 3000 \\
\hline
\end{tabular}

Table 6.2: Details of MCMC Run for All Calibrations

All calibrations converged based on the aforementioned Batch Means test with a $95 \%$ 
confidence interval. Upon convergence, 50 randomly selected converged results are plotted together with experimental data to gauge how effective a specific model can be used model the GB effect. Figure 6.4 - Figure 6.8 show results for selective model versions. The semitransparent blue curves are randomly selected model results based on posterior parameter distributions, and the colored curves are experimental measurement from GBs of different angels. For the profiles above, Model V0 is similar to the Gouy-Chapman case, with no de-
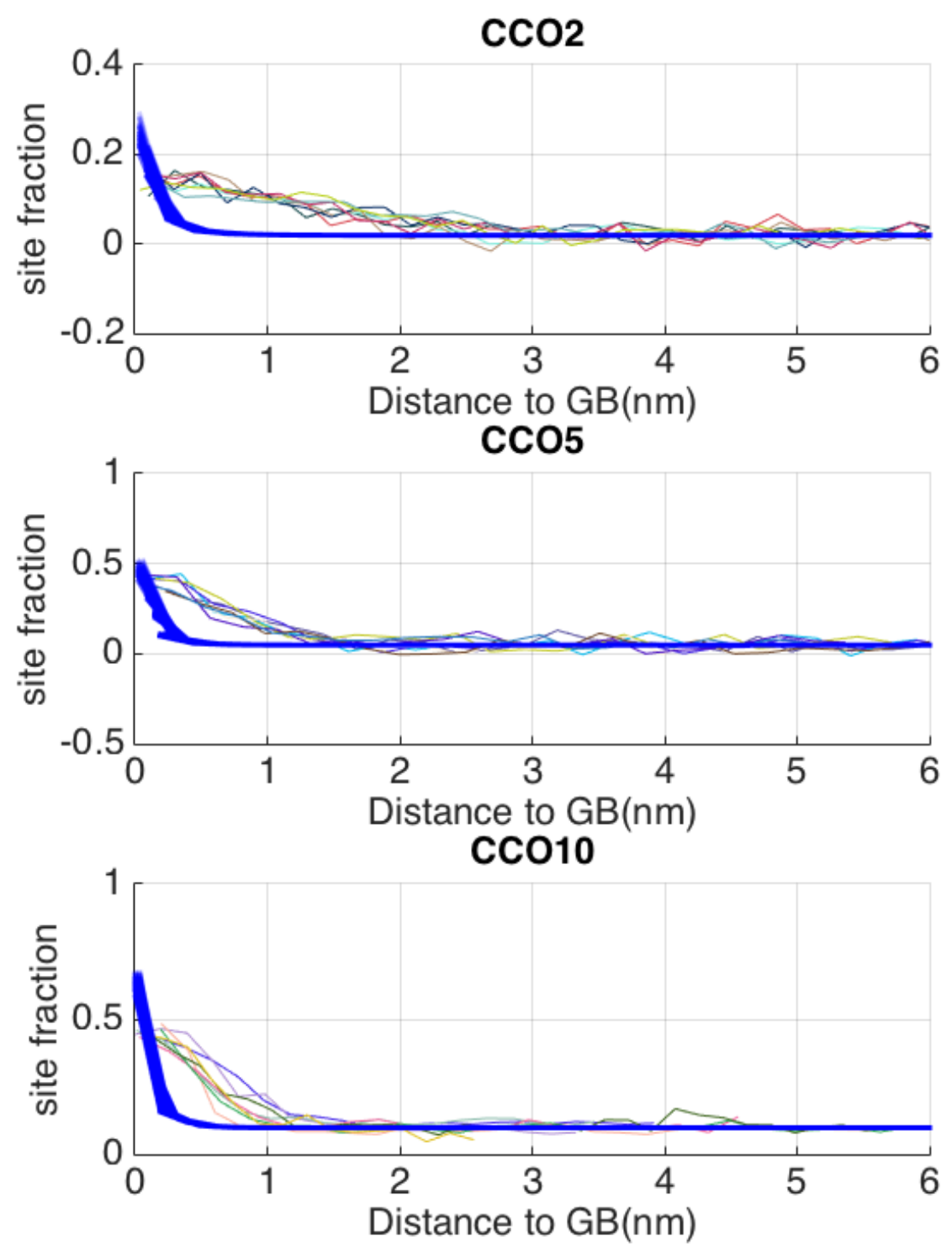

Figure 6.4: Calibration Results for Baseline Model V0 

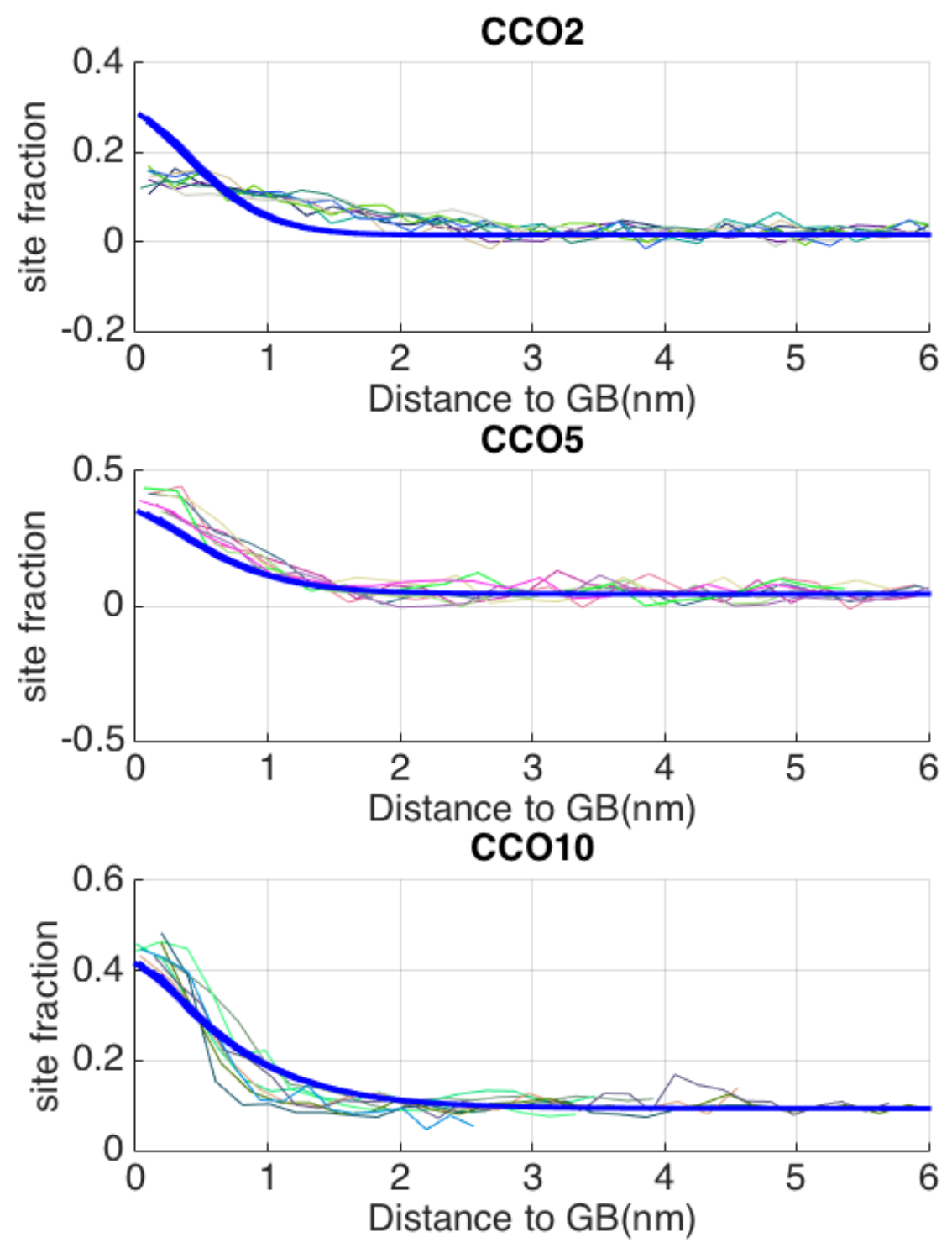

Figure 6.5: Calibration Results for Model V7

fect interactions or gradient effects. Model V7 includes dopant self-interactions and dopant gradient energy effect with no concentration dependence.Model V8 includes vacancy selfinteractions and vacancy gradient energy effect with no concentration dependence. Model V19 includes self-interactions of both dopant and vacancy up to the second order, the interaction between the two, and the corresponding gradient energy effects with no concentration dependence. Model V20 includes more complex gradient energy effects with concentration 

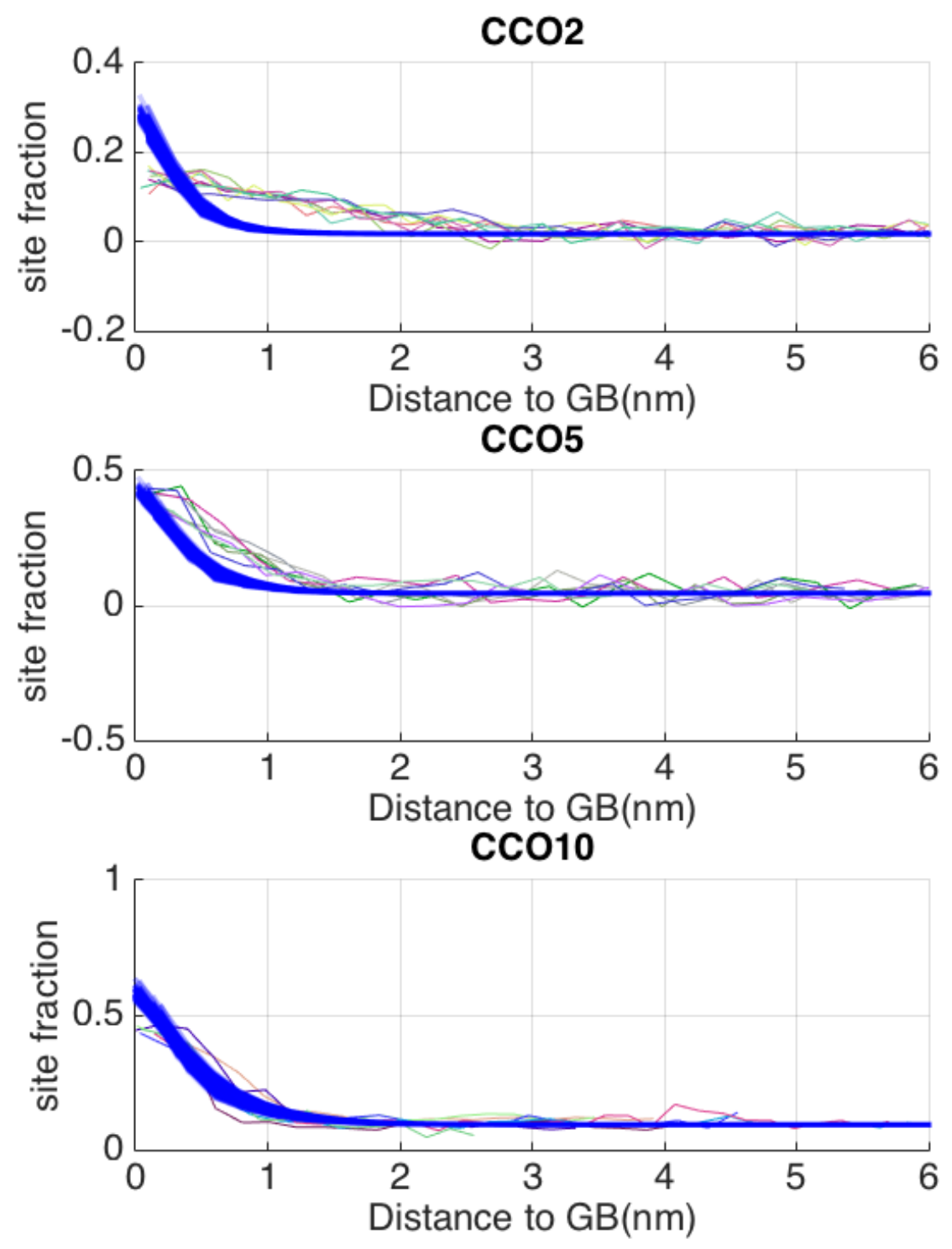

Figure 6.6: Calibration Results for Model V8

dependence for both dopant and vacancy in addition to what model V19 already incorporated. These results clearly revealed how this general framework becomes more effective after significant effects have been systematically included. A more quantified and in-depth model comparison will be done in the next section. For each model version, the posterior distributions of model parameters are summarized in Table 6.3. 

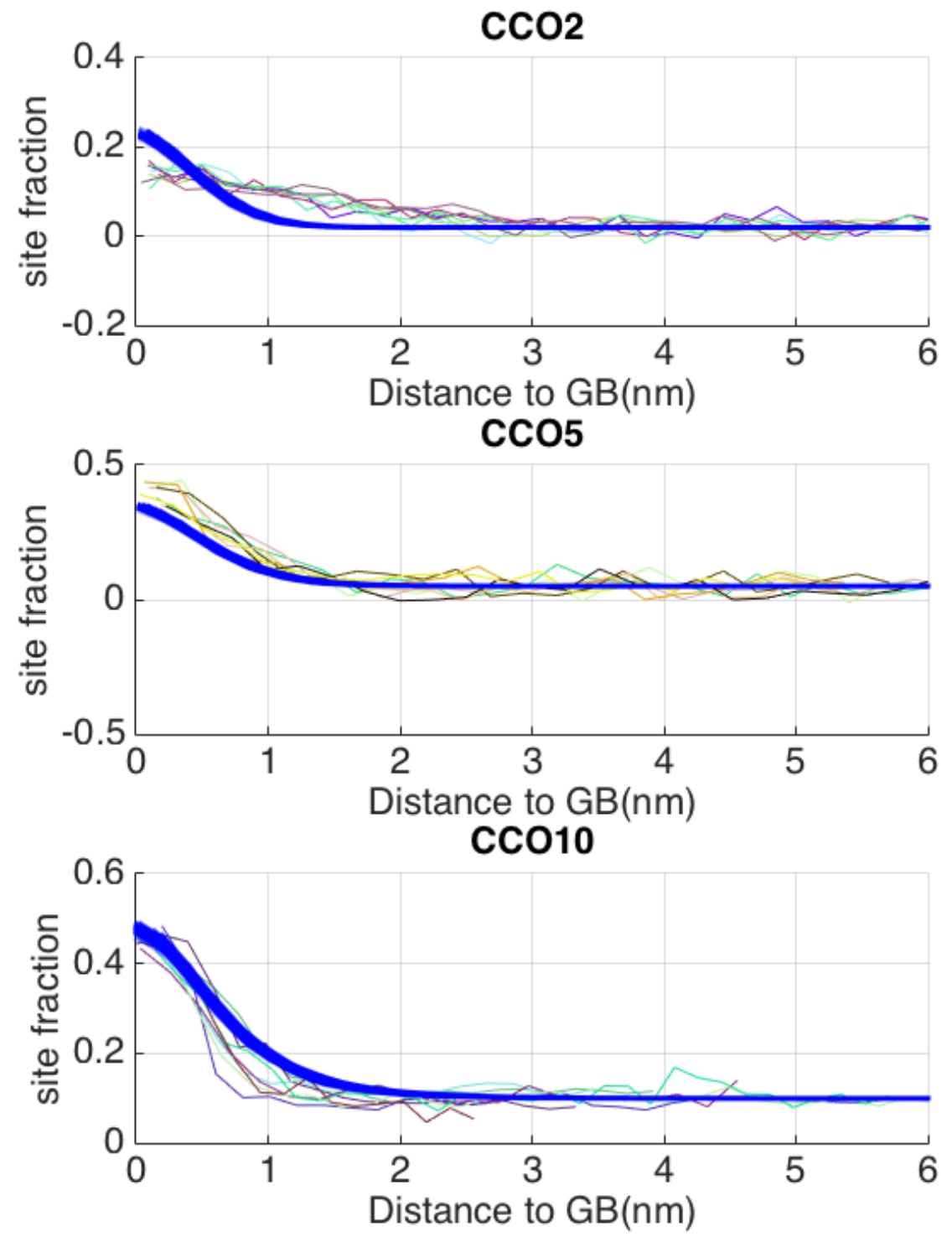

Figure 6.7: Calibration Results for Model V19 

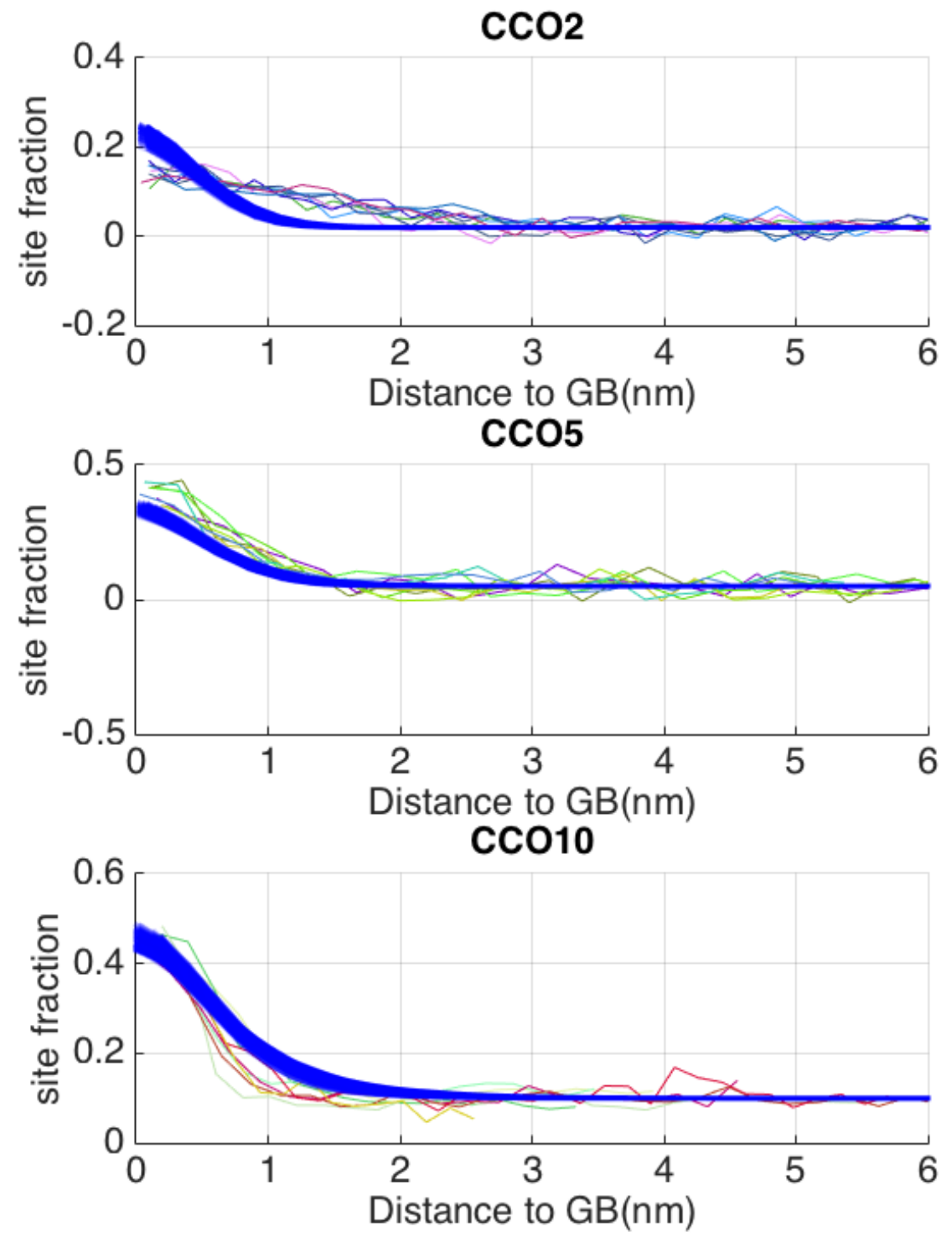

Figure 6.8: Calibration Results for Model V20 


\begin{tabular}{|c|c|c|c|c|c|c|c|c|c|c|c|c|}
\hline \multirow{2}{*}{ Param } & \multicolumn{2}{|c|}{$n_{\mathrm{o}} \times 10^{5}\left(\mathrm{~mol} / \mathrm{m}^{2}\right)$} & \multicolumn{2}{|c|}{$f_{y}(\mathrm{eV})$} & \multicolumn{2}{|c|}{$f_{y v}(\mathrm{eV})$} & \multicolumn{2}{|c|}{$f_{v}(\mathrm{eV})$} & \multicolumn{2}{|c|}{$c_{y}\left(\mathrm{eV}-\mathrm{nm}^{2}\right)$} & \multicolumn{2}{|c|}{$c_{v}\left(\mathrm{eV}-\mathrm{nm}^{2}\right)$} \\
\hline & $\mathrm{m}$ & $\mathrm{s}$ & $\mathrm{m}$ & $\mathrm{s}$ & $\mathrm{m}$ & $\mathrm{s}$ & $\mathrm{m}$ & $\mathrm{s}$ & $\mathrm{m}$ & s & $\mathrm{m}$ & s \\
\hline V0 & 0.43 & 0.02 & & & & & & & & & & \\
\hline V1 & 0.37 & 0.03 & 0.54 & 0.27 & & & & & & & & \\
\hline V2 & 0.37 & 0.03 & & & & & 0.56 & 0.26 & & & & \\
\hline V3 & 0.35 & 0.02 & & & -0.43 & 0.27 & & & & & & \\
\hline $\mathrm{V} 4$ & 0.35 & 0.02 & 0.53 & 0.25 & & & 0.54 & 0.25 & & & & \\
\hline V5 & 0.38 & 0.02 & & & -0.47 & 0.26 & 0.56 & 0.25 & & & & \\
\hline V6 & 0.37 & 0.03 & 0.54 & 0.24 & -0.48 & 0.27 & & & & & & \\
\hline V7 & 1.24 & 0.04 & 0.55 & 0.25 & & & & & 1.18 & 0.12 & & \\
\hline V8 & 1.83 & 0.06 & & & & & 0.55 & 0.25 & & & 0.96 & 0.03 \\
\hline V9 & 0.36 & 0.01 & 0.55 & 0.25 & -0.46 & 0.26 & 0.56 & 0.25 & & & & \\
\hline V10 & 0.38 & 0.13 & 0.55 & 0.24 & & & 0.55 & 0.25 & 0.28 & 0.12 & & \\
\hline V11 & 1.77 & 0.02 & 0.54 & 0.25 & & & 0.54 & 0.24 & & & 0.98 & 0.01 \\
\hline V12 & 0.39 & 0.13 & 0.54 & 0.25 & -0.53 & 0.26 & & & 0.28 & 0.13 & & \\
\hline V13 & 1.74 & 0.03 & & & -0.53 & 0.27 & 0.56 & 0.24 & & & 0.98 & 0.01 \\
\hline V14 & 0.80 & 0.11 & 0.53 & 0.24 & & & 0.54 & 0.23 & 0.10 & 0.003 & 0.12 & 0.01 \\
\hline V15 & 1.36 & 0.03 & 0.55 & 0.25 & -0.54 & 0.27 & 0.55 & 0.25 & 1.40 & 0.12 & & \\
\hline V16 & 2.04 & 0.03 & 0.55 & 0.25 & -0.52 & 0.27 & 0.55 & 0.24 & & & 0.99 & 0.01 \\
\hline V17 & 1.99 & 0.05 & 0.55 & 0.25 & -0.44 & 0.26 & 0.53 & 0.24 & 0.46 & 0.10 & 0.57 & 0.16 \\
\hline \multirow{2}{*}{ V18 } & \multirow{2}{*}{1.36} & \multirow{2}{*}{0.03} & 0.54 & 0.25 & \multirow{2}{*}{-0.54} & \multirow{2}{*}{0.27} & & & \multirow{2}{*}{1.41} & \multirow{2}{*}{0.11} & & \\
\hline & & & 0.46 & 0.23 & & & & & & & & \\
\hline \multirow{2}{*}{ V19 } & \multirow{2}{*}{2.02} & \multirow{2}{*}{0.05} & 0.55 & 0.25 & \multirow{2}{*}{-0.44} & \multirow{2}{*}{0.27} & 0.55 & 0.25 & \multirow{2}{*}{0.46} & \multirow{2}{*}{0.10} & \multirow{2}{*}{0.58} & \multirow{2}{*}{0.15} \\
\hline & & & 0.63 & 0.24 & & & 0.59 & 0.25 & & & & \\
\hline \multirow{2}{*}{ V20 } & \multirow{2}{*}{2.29} & \multirow{2}{*}{0.16} & 0.53 & 0.25 & \multirow{2}{*}{-0.52} & \multirow{2}{*}{0.27} & 0.54 & 0.25 & 0.63 & 0.19 & 0.70 & 0.19 \\
\hline & & & 0.55 & 0.25 & & & 0.53 & 0.25 & 0.44 & 0.32 & 0.28 & 0.21 \\
\hline \multirow{2}{*}{ V21 } & ית 2 & A 18 & 0.56 & 0.25 & -0.48 & 0.26 & 0.61 & 0.24 & 0.57 & 0.15 & 0.72 & 0.16 \\
\hline & 2.20 & 0.10 & 0.63 & 0.24 & -0.42 & 0.26 & 0.53 & 0.24 & 0.33 & 0.24 & 0.24 & 0.18 \\
\hline
\end{tabular}

Table 6.3: Statistics of Posterior Distributions 
Where $\mathrm{m}$ and $\mathrm{s}$ in the table header stands for mean and standard deviation, respectively. $f_{y}, f_{v}$ indicate self-interaction energy of dopant and vacancy respectively, with a unit of $\mathrm{eV}, f_{y v}$ indicates dopant-vacancy interaction in $\mathrm{eV} . c_{y}, c_{v}$ represent the gradient energy coefficient of dopant and vacancy respectively. Multiple rows in one parameter field (for example, the field of $f_{y}$ and $f_{v}$ for model V19) represent multiple orders of the corresponding discrepancy functions in the formulation (row 1 corresponds to the first order, row 2 corresponds to the second order). From the statistics of these posterior distributions summarized in the table, consistency of distributions for these models and changes in distributions with the incorporation of gradient energy coefficients are clearly shown. The posterior distributions of $f_{y}$ for all model versions unified at a distribution with a mean value around $0.54 \mathrm{eV}$ and a standard deviation around $0.25 \mathrm{eV}$. The posterior distributions of $f_{v}$ for all model versions unified at a distribution with a mean value around $0.55 \mathrm{eV}$ and a standard deviation

around $0.25 \mathrm{eV}$. These provide strong evidences for the reference values of $f_{y}$ and $f_{v}$. The posterior distributions of $n_{o}$ across all model versions differ depending on the incorporation of gradient energy distributions in the model structure. With no inclusion of gradient energy contributions, the mean value of its posterior falls on the lower range of around $0.37 \times 10^{-5}$, as for model V0-V6 and model V9. Once any gradient energy contributions are incorporated, the mean value of its posterior falls on the higher range of $>1.24 \times 10^{-5}$, as for model V7,V8, V11, V13-V20. Model V10 and V12, however, didn't conform to this observation.

\subsection{Model Comparison Using the Bayes Factor}

Bayes factor is a method of hypothesis testing using likelihood probabilities of two competing models. It quantifies the support of one model M1 over M2 given data y by evaluating the probability of each model fitting data y. The formulation of Bayes factor for comparing model M1 and M2 is represented in Equation 6.35[123].

$$
B_{12}=\frac{\int p_{1}(y \mid \beta) \pi_{1}(\beta) d \beta}{\int p_{2}(y \mid \theta) \pi_{2}(\theta) d \theta}
$$


Where $\beta$ and $\theta$ are model parameters, $\pi_{1}$ and $\pi_{2}$ are the priors of model M1 and M2, respectively. As can be seen, the Bayes factor doesn't depend on any single set of parameters as it integrates over the space of all model parameters. Additionally, this enables the consideration of including penalty for too complicated model structures, preventing overfitting. As a robust and effective way for model comparison, the Bayes factor approach is adopted to select the optimal model given data in this study. The estimation of the nominator and denominator is done via posterior simulation using the Laplace method. At every point $\theta_{0}$, an estimate of the posterior density can be obtained. Thus, $\left.\int p_{(} y \mid \theta\right) \pi(\theta) d \theta$ can be estimated with $\int L(\theta) \pi(\theta) d \theta$, the likelihood $L$ equals $\left.p_{(} y \mid \theta\right)$. The Laplace method uses a normal estimate of the posterior density with an adaptive kernel estimate.

The natural logarithms of the Bayes factor values of the afore-mentioned models relative to the baseline model V0 are represented in Figure 6.9.

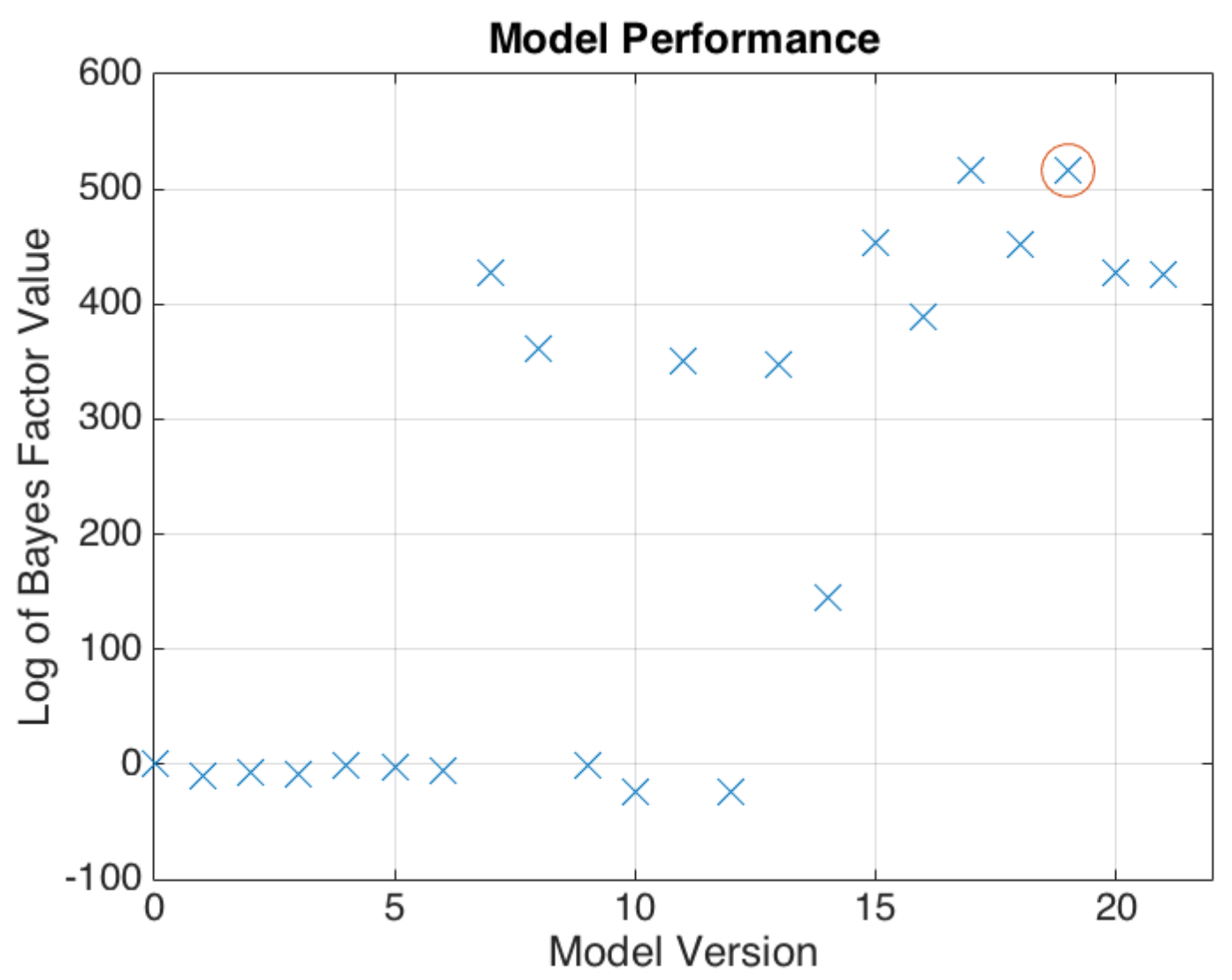

Figure 6.9: Model Performance Quantified by the Bayes Factor 
The Bayes factor in the figure provided a rigorous and reliable evaluation of the effectiveness of these models. In terms of the Bayes factor, model V1-V6 and model V9 showed lower values close to the baseline model V0, indicating that the sole consideration of defect interactions failed to represent the grain boundary effect. The performances of these models are even worse than the traditional Gouy-Chapman model when reproducing the experimental data. The Bayes factor of model V4-V6 and model V9 are higher than that of model V1-V3, which is reasonable as including multiple interaction energies are more effective than including just one type of defect interaction.

With the incorporation of gradient energy distribution pertaining to one type of defect (dopant or vacancy), the model performance significantly improves, proving the necessity of considering this effect in treating non-dilute systems. These models are model V7,V8,V11, and V13. The fact that model V7 overperformed model V8 indicates that the dopant gradient energy is more significant than the vacancy gradient energy.

There are two exceptions for model versions with dopant gradient energy considered: model V10 and V12. Model V10 included $f_{v}$ while Model V12 included $f_{y v}$ other than the terms of $f_{y}$ and $c_{y}$. This dictates that incorporating just one type of defect interaction other than $f_{y}$ is far less effective compared with models without this defect interaction as far as $c_{y}$ is considered. This conclusion is drawn as the Bayes factor of model V16 fell at a much higher value compared with that of the model V10 and V12.

The similar observation is not present for models incorporating $f_{v}$ amd $c_{v}$. Adding just one type of defect interaction other than $f_{v}$ doesn't make the model perform worse, as model V11 and V13 performed reasonably well comparing with model V8. However, the Bayes factor of model V8 is indeed higher than that of model V11 and V13, indicating that adding just one type of defect interaction other than $f_{v}$ is not effective as far as $c_{v}$ is considered. Since the Bayes factor of model V14 is lower than that of model V11, it can be concluded that adding more terms to a model is not guaranteed to improve model performance. As one term of $c_{y}$ is added, if the corresponding interaction between dopant 
ions and oxygen vacancies is not incorporated, the model actually performs worse compared with a version without $c_{y}$.

The model performance of model V15 is better than model V7, and that of model V16 is better than model V8. This verifies the expectation that when multiple defect interactions other than the one related with the gradient energy source are incorporated, the corresponding model performs better.

The fact that model V7 and V15 performs better than model V8 and model V16 respectively, dictates that the effect of dopant gradient energy contribution is more significant than that of the vacancy.

Among all the calibrated models, model V19 (as highlighted in Figure 6.9) yields the highest Bayes factor with 2 orders of discrepancy functions for self-interaction of dopant and vacancy, 1 order of discrepancy function for dopant-vacancy interaction, and constant coefficient for gradient energies of dopant and vacancies. This structure reveals the significance of all interactions and gradient energies. The posterior distributions of all corresponding parameters are within physically reasonable ranges. This makes model V19 the optimal option for modeling $\mathrm{CCO}$ of different concentrations.

Taking Model V19 as an example, setting parameters to the mean value of the posterior distributions, the defect interaction energies with respect to defect composition are shown in the figures below. For both $f_{y}$ and $f_{v}$, there are two orders of function components as shown in Figure 6.10, as a result of the BSS-ANOVA basis functions being multiplied by the corresponding coefficients. The total function form of these two are displayed in Figure 6.11. the function forms of $f_{y}$ and $f_{v}$ are very similar as the posteriors of the corresponding parameters have similar mean values. Figure 6.12 displays the functional form of $f_{y v}$ with only a first order component for model V19. All these function forms of the defect interaction energies are revealed through calibrating the model with the experimental data. The defect interaction energies are shifted based on the Const term in Equation 6.2 in order to maintain zero defect interaction energies given zero defect concentrations. 

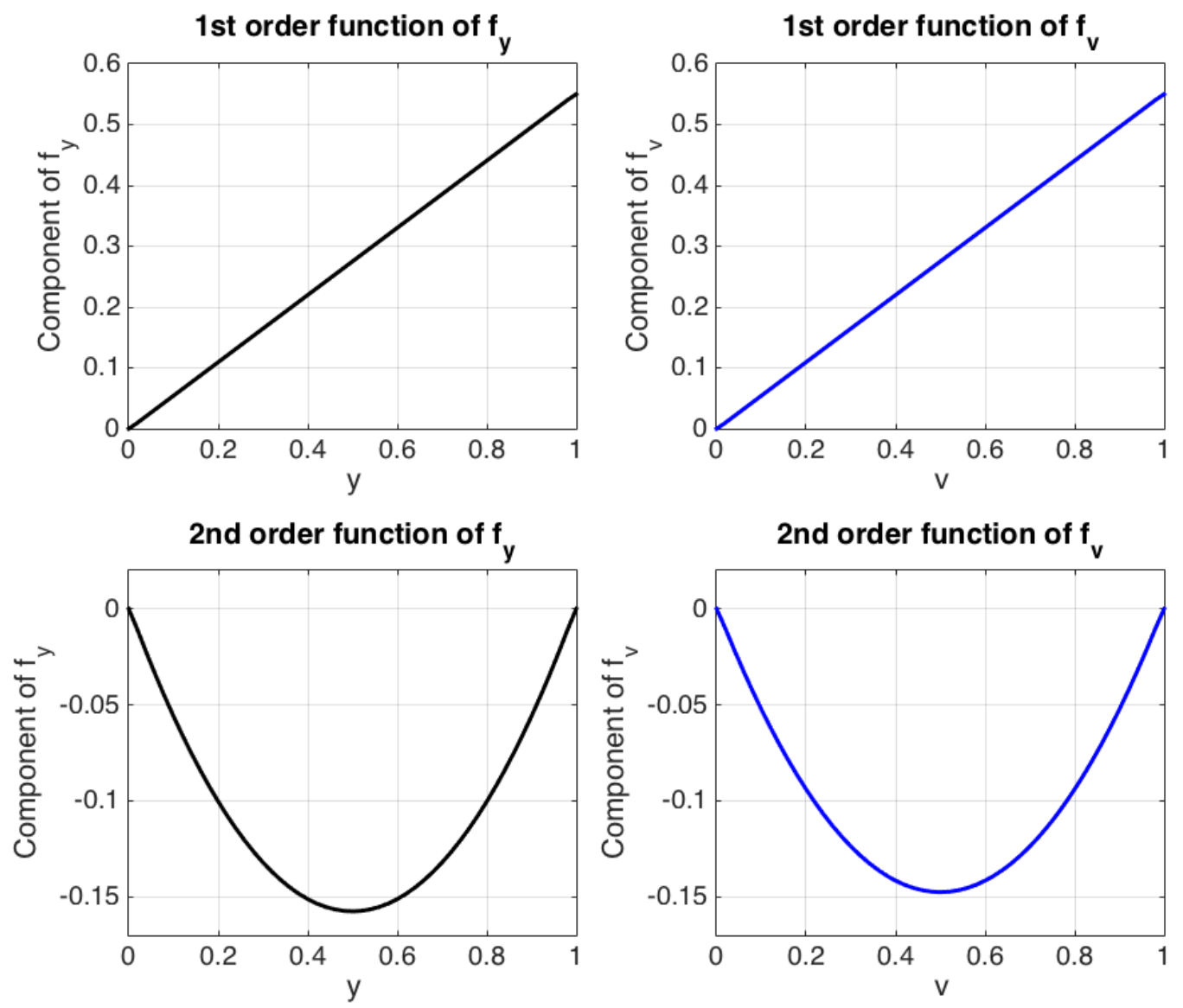

Figure 6.10: Function Components of $f_{y}$ and $f_{v}$ 


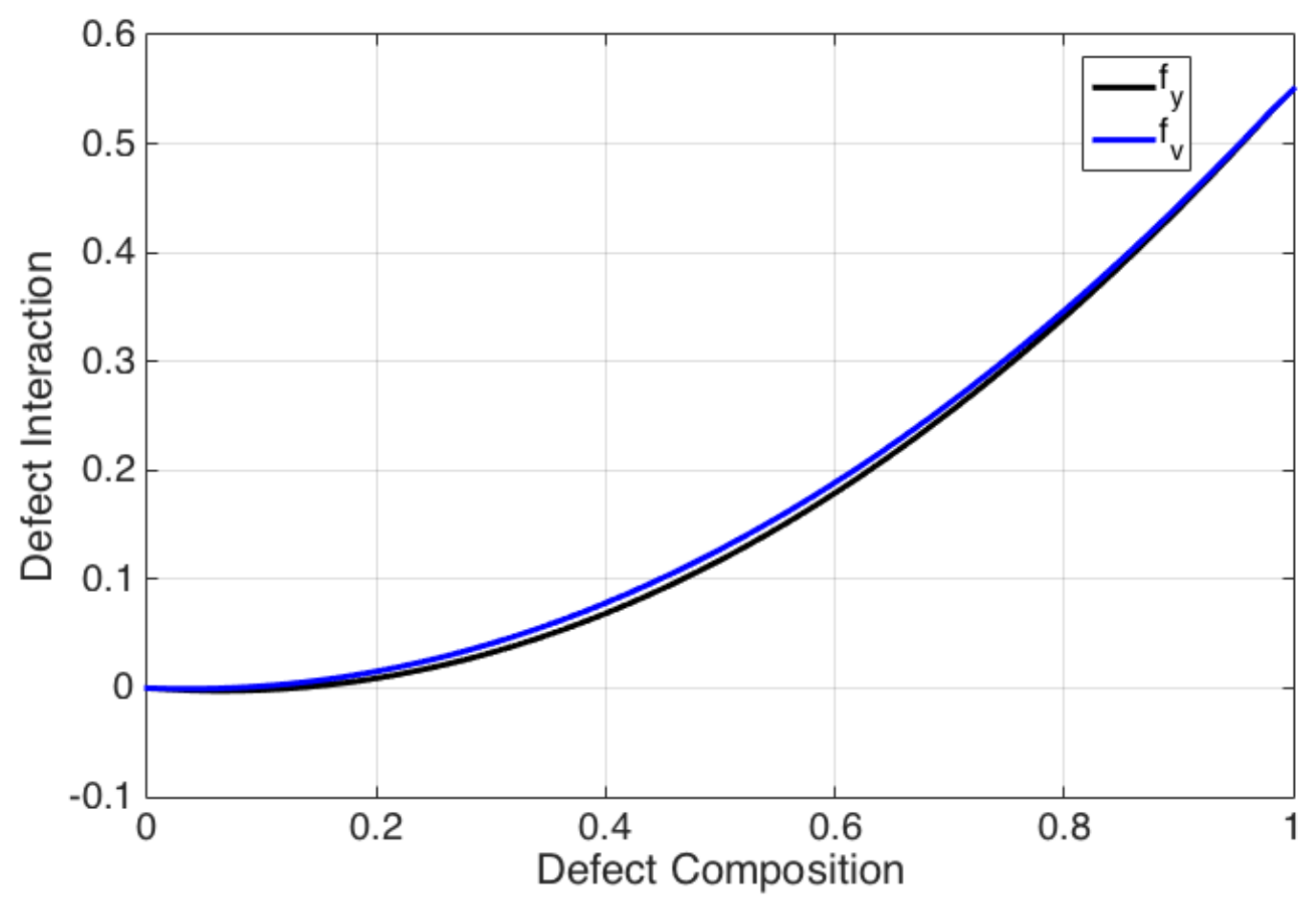

Figure 6.11: Function form of $f_{y}$ and $f_{v}$

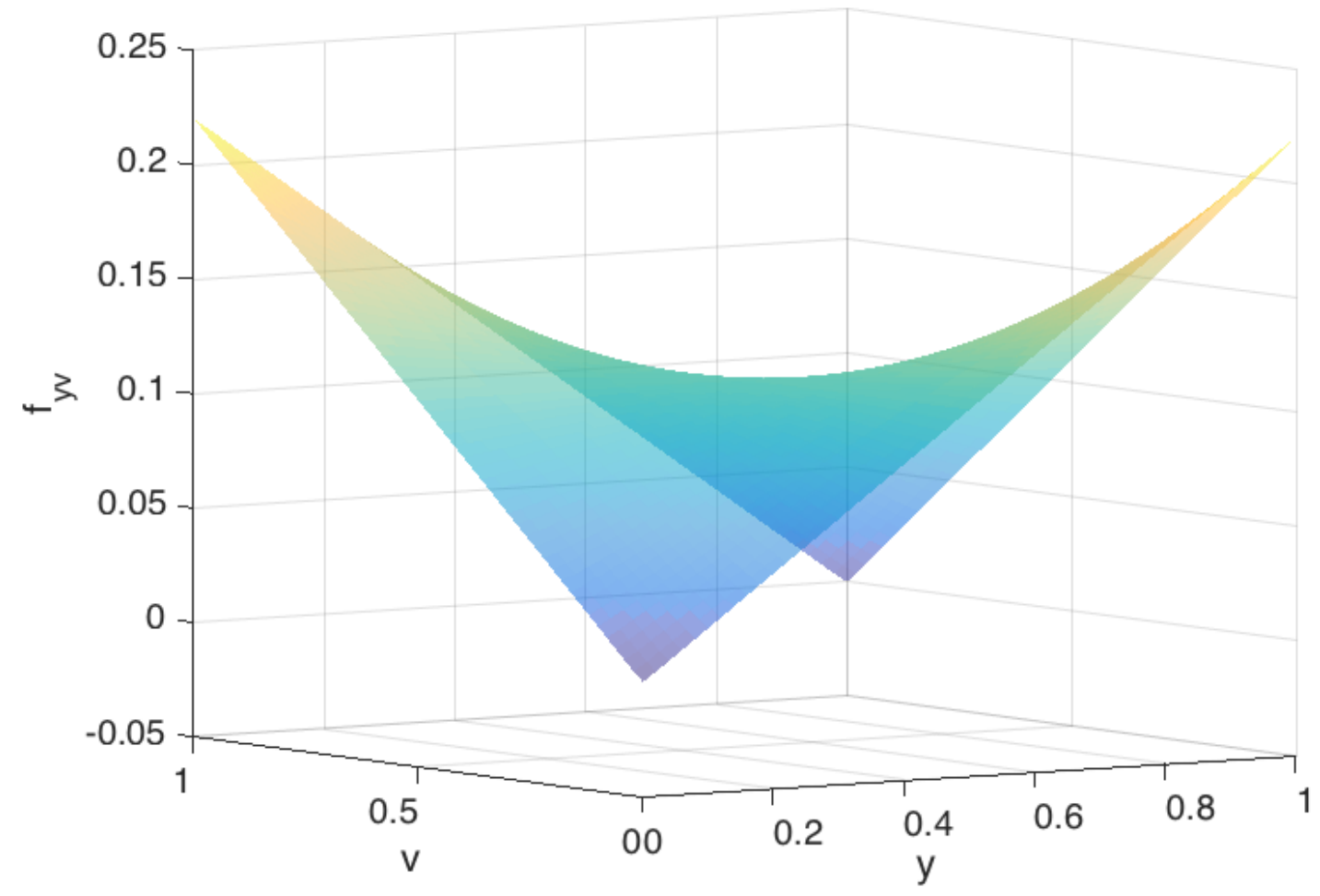

Figure 6.12: Function form of $f_{y v}$ 
In Figure 6.13, the vacancy profiles of $\mathrm{CCO} 2, \mathrm{CCO} 5$ and $\mathrm{CCO} 10$ calculated with model V19 show accumulation near the GB. The presented figure includes 50 vacancy profiles for each concentration, calculated based on randomly selected parameter sets from the posteriors. This confirms the capability of the model in reproducing co-accumulation of vacancies and dopants as observed by APT.
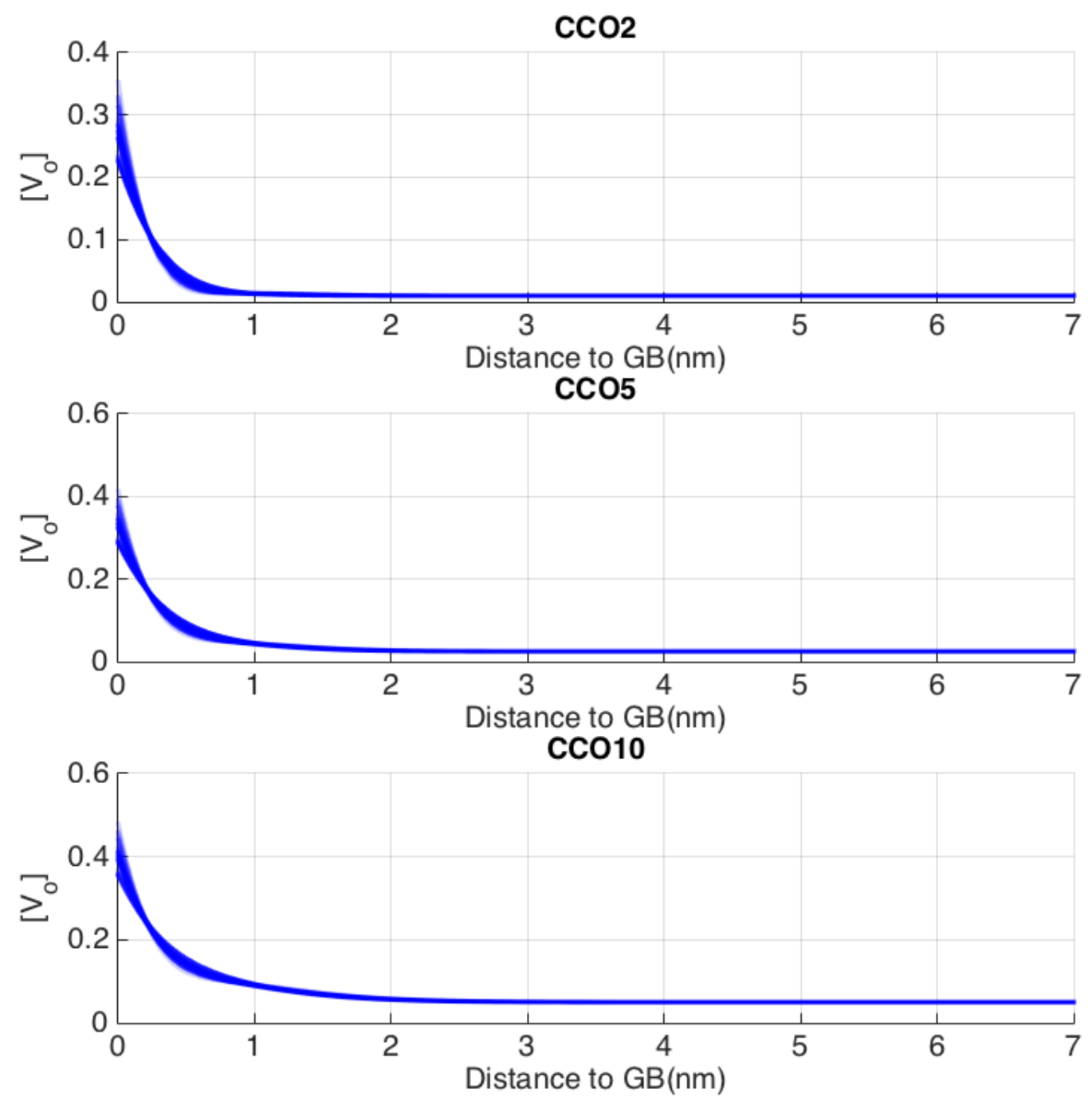

Figure 6.13: Vacancy Profiles near the GB as Evaluated with Model V19 


\section{CHAPTER 7}

\section{CONCLUSIONS}

A kinetic model was developed, based on the generalized Poisson-Cahn theory, to simulate defect profile development upon thermal annealing of oxygen-conducting electrolytes. GDC with dopant levels of $20 \%, 1 \%$ and $0.1 \%$ were evaluated. At $1300^{\circ} \mathrm{C}$, model results were able to predict the complex behavior of dopant profile development, from the initial segregation accompanied by depletion near the GB, followed by broadening of the segregation and depletion zones - which produced long-range effect reaching more then $10 \mathrm{~nm}$ into the bulk - to the final equilibrium profile of dopant segregation in a region of only a few nanometers. The kinetic process differed in the details for GDC of different dopant levels, as did the time to approach equilibrium. Simulations were performed for multiple temperature levels below $1300^{\circ} \mathrm{C}$; with slower cation diffusivity, time to equilibrium was significantly longer, reaching 59 hrs. at $850^{\circ} \mathrm{C}$ for GDC with $20 \%$ dopant concentration. The quench temperature, at which the dopant profile seems to be frozen on the timescale of typical annealing processes, was found to be around $900{ }^{\circ} \mathrm{C}$.

Using the frozen-in temperature concluded from the kinetic model, a Poisson-Cahn model treating the realistic case of a restricted equilibrium situation (with dopant profile frozen at a high temperature and vacancies equilibrated at the measurement temperature) is constructed. With model results of defect distributions near the GB, the corresponding bulk and GB conductivities are calculated using the Nakayama-Martin conductivity model. Particle swarm optimization is used to find a good fit between the model calculated conduc-

tivity data and the experimental data reported in literature. With the same set of physically reasonable model parameters, conductivity predictions across a broad dopant concentration range conformed well with experimental data. The drop in total conductivity as dopant 
concentration increases is also reproduced by the model. Furthermore, the defect profiles predicted by the Poisson-Cahn model showed segregation layers of several nanometers especially at high dopant concentrations, a feature that can not be obtained with traditional space charge theories like Gouy-Chapman. The characteristic length from the Poisson-Cahn model matched that from the Gouy-Chapman model for dilute systems, while diverged from the GC results for concentrated systems. This provided a strong evidence for the applicability of Poisson-Cahn models for material systems of all concentrations.

A two-step equilibration model is used to calculated defect profiles near GBs of NDC10 and NDC30. Bayesian calibration is employed to explore the model parameter spaces that can reproduce defect co-accumulation The model is calibrated with experimental data and has been shown to be able to reproduce defect co-accumulation near GBs as observed by APT. The converged posteriors for NDC10 and NDC30 are obtained separately in order to achieve calibration results that can cover the measurements well, suggesting that concentration dependence of model parameters including defect interactions and gradient energies existed in this model version. These parameters of NDC10 model show higher averages in absolute value when compared with those of NDC30, indicating possible phase transformations as concentration increases from $10 \%$ to $30 \%$. The differed posterior distributions of models pertaining to NDC10 and NDC30 therefore called for the development of a model framework that can unify parameter spaces across different concentrations.

Therefore, the last part of this dissertation is dedicated to develop a unified PoissonCahn framework capable of modeling complex concentration-dependence of defect interactions and gradient energy coefficients with physically reasonable parameter spaces that are unified for solutions of different concentrations. This is realized through the adoption of discrepancy function forms of the BSS-ANOVA framework into the free energy functional of the material system under study. The basis functions spanning from the main effect to higher-order interactions provide powerful nonparametric ways to model unknown parameter dependences on defect concentrations. With this framework, a series of models are con- 
structed from the simplistic of Gouy-Chapman case to highly-complicated model structures that consider two-way interactions of defect interactions and gradient energy contributions. Bayesian calibration is employed to calibrate each model version with EELS measurements of dopant profiles near multiple GBs of CCO2, CCO5 and CCO10. This is essentially a data-driven approach to allow the experimental data dictate what are the model structure and parameter distributions that can reproduce the experimental measurements. The Bayes factor is used as a reliable guide to compare model performances. The best model fit and posterior distributions are obtained where that is a peak in the Bayes factor values as the model structure further complicates. The model structure with all defect interactions (two orders of basis functions for defect self-interactions and one order of basis function for dopant-vacancy interaction) and gradient energy contributions (constant gradient energy coefficients for both defects) are found to the most effective among all for the material system of CCO with 3 different concentrations. This process also helped to reveal the significance of each factor incorporated into the model. Gradient energy coefficients are found to play a key role in reproducing defect profiles near GBs, the effect of which is enhanced with the incorporation of multiple defect interactions.

The Poisson-Cahn methodology developed in this dissertation, through demonstrations with different material systems, has proven its broad applicability in solid ionic materials with any dopant concentration, especially in concentrated systems. This methodology revolutionized the application of space charge theories in solid state ionics community by replacing traditional theories in the regime of concentrated material systems. Key experimental observations such as extended defect segregation zone and defect co-accumulation in concentrated systems were predicted correctly by this theory, which are impossible for traditional theories. The final development of the unified Poisson-Cahn framework, has quantitively demonstrated the improvement in model capability when progressing from traditional Gouy-Chapman theory to Poisson-Cahn formulations. The parameter spaces identified with Bayesian calibration unify well with ranges found in past literature. This framework also 
serve as the solid ground for further model development to include more species, incorporate more complex interaction and gradient effects, and to predict properties of more material systems once more data is available for model building. 


\section{BIBLIOGRAPHY}

[1] Curtis B Storlie, William A Lane, Emily M Ryan, James R Gattiker, and David M Higdon. Calibration of computational models with categorical parameters and correlated outputs via bayesian smoothing spline anova. Journal of the American Statistical Association, 110(509):68-82, 2015.

[2] William J Bowman, Madeleine N Kelly, Gregory S Rohrer, Cruz A Hernandez, and Peter A Crozier. Enhanced ionic conductivity in electroceramics by nanoscale enrichment of grain boundaries with high solute concentration. Nanoscale, 9(44):17293-17302, 2017.

[3] A.Boudghene Stambouli and E Traversa. Solid oxide fuel cells (sofcs): a review of an environmentally clean and efficient source of energy. Renewable and Sustainable Energy Reviews, 6(5):433 - 455, 2002.

[4] James Hansen, Reto Ruedy, Mki Sato, and Ken Lo. Global surface temperature change. Reviews of Geophysics, 48(4), 2010.

[5] S.P.S. Badwal. Grain boundary resistivity in zirconia-based materials: effect of sintering temperatures and impurities. Solid State Ionics, 76(1):67 - 80, 1995.

[6] Michael C Martin and Martha L Mecartney. Grain boundary ionic conductivity of yttrium stabilized zirconia as a function of silica content and grain size. Solid State Ionics, 161(12):67 - 79, 2003.

[7] Sangtae Kim and Joachim Maier. On the conductivity mechanism of nanocrystalline ceria. Journal of the Electrochemical society, 149(10):J73-J83, 2002.

[8] R.A. De Souza. The formation of equilibrium space-charge zones at grain boundaries in 
the perovskite oxide srtio3. Physical Chemistry Chemical Physics, 11(43):9939-9969, 2009.

[9] Makoto Aoki, Yet-Ming Chiang, Igor Kosacki, L. Jong-Ren Lee, Harry Tuller, and Yaping Liu. Solute segregation and grain-boundary impedance in high-purity stabilized zirconia. Journal of the American Ceramic Society, 79(5):1169-1180, 1996.

[10] Xin Guo and Rainer Waser. Electrical properties of the grain boundaries of oxygen ion conductors: acceptor-doped zirconia and ceria. Progress in Materials Science, 51(2):151-210, 2006.

[11] Naoya Shibata, Yuichi Ikuhara, Fumiyasu Oba, Takahisa Yamamoto, and Taketo Sakuma. Atomic structure and solute segregation of a $=3,[110] / 111$ grain boundary in an yttria-stabilized cubic zirconia bicrystal. Philosophical Magazine Letters, 82(7):393-400, 2002.

[12] David S. Mebane and Roger A. De Souza. A generalised space-charge theory for extended defects in oxygen-ion conducting electrolytes: from dilute to concentrated solid solutions. Energy Environ. Sci., 8:2935-2940, 2015.

[13] David S. Mebane. A variational approach to surface cation segregation in mixed conducting perovskites. Computational Materials Science, 103:231 - 236, 2015.

[14] P.J Scanlon, R.A.M Bink, F.P.F van Berkel, G.M Christie, L.J van IJzendoorn, H.H Brongersma, and R.G van Welzenis. Surface composition of ceramic cegdoxide. Solid State Ionics, 112(12):123 - 130, 1998.

[15] William J Bowman, Jiangtao Zhu, Renu Sharma, and Peter A Crozier. Electrical conductivity and grain boundary composition of gd-doped and gd/pr co-doped ceria. Solid State Ionics, 272:9-17, 2015. 
[16] Yuanyuan Lei, Yasuo Ito, Nigel D Browning, and Terry J Mazanec. Segregation effects at grain boundaries in fluorite-structured ceramics. Journal of the American Ceramic Society, 85(9):2359-2363, 2002.

[17] Hajime Hojo, Teruyasu Mizoguchi, Hiromichi Ohta, Scott D Findlay, Naoya Shibata, Takahisa Yamamoto, and Yuichi Ikuhara. Atomic structure of a ceo2 grain boundary: the role of oxygen vacancies. Nano letters, 10(11):4668-4672, 2010.

[18] Wonyoung Lee, Hee Joon Jung, Min Hwan Lee, Young-Beom Kim, Joong Sun Park, Robert Sinclair, and Fritz B Prinz. Oxygen surface exchange at grain boundaries of oxide ion conductors. Advanced Functional Materials, 22(5):965-971, 2012.

[19] Francesco Giannici, Giuliano Gregori, Chiara Aliotta, Alessandro Longo, Joachim Maier, and Antonino Martorana. Structure and oxide ion conductivity: Local order, defect interactions and grain boundary effects in acceptor-doped ceria. Chemistry of Materials, 26(20):5994-6006, 2014.

[20] Kepeng Song, Herbert Schmid, Vesna Srot, Elisa Gilardi, Giuliano Gregori, Kui Du, Joachim Maier, and Peter A van Aken. Cerium reduction at the interface between ceria and yttria-stabilised zirconia and implications for interfacial oxygen non-stoichiometry. APL Materials, 2(3):032104, 2014.

[21] David R Diercks, Jianhua Tong, Huayang Zhu, Robert Kee, George Baure, Juan C Nino, Ryan O'Hayre, and Brian P Gorman. Three-dimensional quantification of composition and electrostatic potential at individual grain boundaries in doped ceria. Journal of Materials Chemistry A, 4(14):5167-5175, 2016.

[22] A Tschöpe, S Kilassonia, and R Birringer. The grain boundary effect in heavily doped cerium oxide. Solid State Ionics, 173(1):57-61, 2004.

[23] D Bingham, PW Tasker, and AN Cormack. Simulated grain-boundary structures and ionic conductivity in tetragonal zirconia. Philosophical Magazine A, 60(1):1-14, 1989. 
[24] Hark B Lee, Friedrich B Prinz, and Wei Cai. Atomistic simulations of grain boundary segregation in nanocrystalline yttria-stabilized zirconia and gadolinia-doped ceria solid oxide electrolytes. Acta Materialia, 61(10):3872-3887, 2013.

[25] Xiao-Dong Zhou, Wayne Huebner, Igor Kosacki, and Harlan U Anderson. Microstructure and grain-boundary effect on electrical properties of gadolinium-doped ceria. Journal of the American Ceramic Society, 85(7):1757-1762, 2002.

[26] Jörg Waldhäusl, Wolfgang Preis, and Werner Sitte. Electrochemical characterization of gadolinia-doped ceria using impedance spectroscopy and dc-polarization. Solid State Ionics, 225:453-456, 2012.

[27] Kurt Lehovec. Space-charge layer and distribution of lattice defects at the surface of ionic crystals. The Journal of Chemical Physics, 21(7):1123-1128, 1953.

[28] Joachim Maier. Physical chemistry of ionic materials: ions and electrons in solids. John Wiley \& Sons, 2004.

[29] Harry L Tuller, Scott J Litzelman, and WooChul Jung. Micro-ionics: next generation power sources. Physical Chemistry Chemical Physics, 11(17):3023-3034, 2009.

[30] Weida Shen, Jun Jiang, Chaoying Ni, Zachary Voras, Thomas P Beebe, and Joshua L Hertz. Two-dimensional vacancy trapping in yttria doped ceria. Solid State Ionics, $255: 13-20,2014$.

[31] Giuliano Gregori, Mona Shirpour, and Joachim Maier. Proton conduction in dense and porous nanocrystalline ceria thin films. Advanced Functional Materials, 23(47):58615867, 2013.

[32] Till Frömling, Herbert Hutter, and Jürgen Fleig. Oxide ion transport in donor-doped pb (zrxti1- x) o3: Near-surface diffusion properties. Journal of the American Ceramic Society, 95(5):1692-1700, 2012. 
[33] Xiangxin Guo and Joachim Maier. Ionically conducting two-dimensional heterostructures. Advanced Materials, 21(25-26):2619-2631, 2009.

[34] Sangtae Kim and Joachim Maier. On the conductivity mechanism of nanocrystalline ceria. Journal of the Electrochemical society, 149(10):J73-J83, 2002.

[35] Sangtae Kim and Joachim Maier. Partial electronic and ionic conduction in nanocrystalline ceria: role of space charge. Journal of the European Ceramic Society, 24(6):1919-1923, 2004.

[36] Mona Shirpour, Behnaz Rahmati, Wilfried Sigle, Peter A van Aken, Rotraut Merkle, and Joachim Maier. Dopant segregation and space charge effects in proton-conducting bazro3 perovskites. The Journal of Physical Chemistry C, 116(3):2453-2461, 2012.

[37] Mona Shirpour, Behnaz Rahmati, Wilfried Sigle, Peter A van Aken, Rotraut Merkle, and Joachim Maier. Dopant segregation and space charge effects in proton-conducting bazro3 perovskites. The Journal of Physical Chemistry C, 116(3):2453-2461, 2012.

[38] William J Bowman, Jiangtao Zhu, Renu Sharma, and Peter A Crozier. Electrical conductivity and grain boundary composition of gd-doped and gd/pr co-doped ceria. Solid State Ionics, 272:9-17, 2015.

[39] Yuanyuan Lei, Yasuo Ito, Nigel D Browning, and Terry J Mazanec. Segregation effects at grain boundaries in fluorite-structured ceramics. Journal of the American Ceramic Society, 85(9):2359-2363, 2002.

[40] Wonyoung Lee, Hee Joon Jung, Min Hwan Lee, Young-Beom Kim, Joong Sun Park, Robert Sinclair, and Fritz B Prinz. Oxygen surface exchange at grain boundaries of oxide ion conductors. Advanced Functional Materials, 22(5):965-971, 2012.

[41] Jihwan An, Joong Sun Park, Ai Leen Koh, Hark B Lee, Hee Joon Jung, Joop Schoonman, Robert Sinclair, Turgut M Gür, and Fritz B Prinz. Atomic scale verification of 
oxide-ion vacancy distribution near a single grain boundary in ysz. Scientific reports, $3: 2680,2013$.

[42] Hark B Lee, Friedrich B Prinz, and Wei Cai. Atomistic simulations of surface segregation of defects in solid oxide electrolytes. Acta Materialia, 58(6):2197-2206, 2010.

[43] David R Diercks, Jianhua Tong, Huayang Zhu, Robert Kee, George Baure, Juan C Nino, Ryan O'Hayre, and Brian P Gorman. Three-dimensional quantification of composition and electrostatic potential at individual grain boundaries in doped ceria. Journal of Materials Chemistry A, 4(14):5167-5175, 2016.

[44] Nele Moelans, Bart Blanpain, and Patrick Wollants. An introduction to phase-field modeling of microstructure evolution. Calphad, 32(2):268-294, 2008.

[45] T Miyazaki, A Takeuchi, T Koyama, and T Kozakai. Computer simulation of phase decomposition in the regular solid solution based upon the cahn-hilliard's non-linear diffusion equation. Materials Transactions, JIM, 32(10):915-920, 1991.

[46] T Miyazaki, A Takeuchi, and T Koyama. Computer simulations of the phase decomposition on cu-co binary alloys based on the non-linear diffusion equation. Journal of materials science, 27(9):2444-2448, 1992.

[47] Hiraku Nishimori and Akira Onuki. Pattern formation in phase-separating alloys with cubic symmetry. Physical Review B, 42(1):980, 1990.

[48] Akira Onuki. Ginzburg-landau approach to elastic effects in the phase separation of solids. Journal of the Physical Society of Japan, 58(9):3065-3068, 1989.

[49] Jingzhi Zhu, Long-Qing Chen, and Jie Shen. Morphological evolution during phase separation and coarsening with strong inhomogeneous elasticity. Modelling and Simulation in Materials Science and Engineering, 9(6):499, 2001. 
[50] Daniel Orlikowski, Celeste Sagui, Andrés Somoza, and Christopher Roland. Large-scale simulations of phase separation of elastically coherent binary alloy systems. Physical Review B, 59(13):8646, 1999.

[51] John W Cahn and John E Hilliard. Free energy of a nonuniform system. iii. nucleation in a two-component incompressible fluid. The Journal of Chemical Physics, 31(3):688699, 1959.

[52] SY Hu and LQ Chen. A phase-field model for evolving microstructures with strong elastic inhomogeneity. Acta materialia, 49(11):1879-1890, 2001.

[53] Y Wang, D Banerjee, CC Su, and AG Khachaturyan. Field kinetic model and computer simulation of precipitation of 112 ordered intermetallics from fcc solid solution. Acta materialia, 46(9):2983-3001, 1998.

[54] DY Li and LQ Chen. Shape evolution and splitting of coherent particles under applied stresses. Acta materialia, 47(1):247-257, 1998.

[55] Yunzhi Wang and Armen Khachaturyan. Effect of antiphase domains on shape and spatial arrangement of coherent ordered intermetallics. Scripta metallurgica et materialia, 31(10):1425-1430, 1994.

[56] V Vaithyanathan and LQ Chen. Coarsening of ordered intermetallic precipitates with coherency stress. Acta Materialia, 50(16):4061-4073, 2002.

[57] Long-Qing Chen. A novel computer simulation technique for modeling grain growth. Scripta Metallurgica et Materialia, 32(1):115-120, 1995.

[58] CE Krill Iii and L-Q Chen. Computer simulation of 3-d grain growth using a phase-field model. Acta materialia, 50(12):3059-3075, 2002. 
[59] Nele Moelans, Bart Blanpain, and Patrick Wollants. Pinning effect of second-phase particles on grain growth in polycrystalline films studied by 3-d phase field simulations. Acta Materialia, 55(6):2173-2182, 2007.

[60] Danan Fan and Long-Qing Chen. Diffusion-controlled grain growth in two-phase solids. Acta materialia, 45(8):3297-3310, 1997.

[61] Danan Fan, Long-Qing Chen, and Shao-Ping P Chen. Numerical simulation of zener pinning with growing second-phase particles. Journal of the American Ceramic Society, 81(3):526-532, 1998.

[62] JS Langer. Models of pattern formation in first-order phase transitions. Directions in Concensed Matter Physics: Memorial Volume in Honor of Shang-Keng Ma. Edited by GRINSTEIN S ET AL. Published by World Scientific Publishing Co. Pte. Ltd., 1986. ISBN\# 9789814415309, pp. 165-186, pages 165-186, 1986.

[63] Ryo Kobayashi. Modeling and numerical simulations of dendritic crystal growth. Physica D: Nonlinear Phenomena, 63(3-4):410-423, 1993.

[64] CW Lan, YC Chang, and CJ Shih. Adaptive phase field simulation of non-isothermal free dendritic growth of a binary alloy. Acta Materialia, 51(7):1857-1869, 2003.

[65] JC Ramirez and C Beckermann. Examination of binary alloy free dendritic growth theories with a phase-field model. Acta Materialia, 53(6):1721-1736, 2005.

[66] CW Lan and YC Chang. Efficient adaptive phase field simulation of directional solidification of a binary alloy. Journal of Crystal Growth, 250(3):525-537, 2003.

[67] Blas Echebarria, Roger Folch, Alain Karma, and Mathis Plapp. Quantitative phasefield model of alloy solidification. Physical Review E, 70(6):061604, 2004.

[68] Adam A Wheeler, GB McFadden, and WJ Boettinger. Phase-field model for solidification of a eutectic alloy. In Proceedings of the Royal Society of London A: Math- 
ematical, Physical and Engineering Sciences, volume 452, pages 495-525. The Royal Society, 1996.

[69] François Drolet, KR Elder, Martin Grant, and JM Kosterlitz. Phase-field modeling of eutectic growth. Physical Review E, 61(6):6705, 2000.

[70] Ingo Steinbach, Franco Pezzolla, Britta Nestler, Markus Seeßelberg, Robert Prieler, Georg J Schmitz, and Joao LL Rezende. A phase field concept for multiphase systems. Physica D: Nonlinear Phenomena, 94(3):135-147, 1996.

[71] Britta Nestler and Adam A Wheeler. Phase-field modeling of multi-phase solidification. Computer Physics Communications, 147(1-2):230-233, 2002.

[72] M Apel, B Boettger, H-J Diepers, and I Steinbach. 2d and 3d phase-field simulations of lamella and fibrous eutectic growth. Journal of crystal growth, 237:154-158, 2002.

[73] Seong Gyoon Kim, Won Tae Kim, Toshio Suzuki, and Machiko Ode. Phase-field modeling of eutectic solidification. Journal of crystal growth, 261(1):135-158, 2004.

[74] John W Cahn and John E Hilliard. Free energy of a nonuniform system. i. interfacial free energy. The Journal of chemical physics, 28(2):258-267, 1958.

[75] Catherine M Bishop, R Edwin García, and W Craig Carter. Effect of charge separation on the stability of large wavelength fluctuations during spinodal decomposition. Acta materialia, 51(6):1517-1524, 2003.

[76] R Edwin García, Catherine M Bishop, and W Craig Carter. Thermodynamically consistent variational principles with applications to electrically and magnetically active systems. Acta Materialia, 52(1):11-21, 2004.

[77] BC Han, A Van der Ven, D Morgan, and G Ceder. Electrochemical modeling of intercalation processes with phase field models. Electrochimica Acta, 49(26):46914699, 2004. 
[78] JE Guyer, WJ Boettinger, JA Warren, and GB McFadden. Phase field modeling of electrochemistry. i. equilibrium. Physical Review E, 69(2):021603, 2004.

[79] JE Guyer, WJ Boettinger, JA Warren, and GB McFadden. Phase field modeling of electrochemistry. ii. kinetics. Physical Review E, 69(2):021604, 2004.

[80] Linyun Liang, Yue Qi, Fei Xue, Saswata Bhattacharya, Stephen J Harris, and LongQing Chen. Nonlinear phase-field model for electrode-electrolyte interface evolution. Physical Review E, 86(5):051609, 2012.

[81] Gogi K Singh, Gerbrand Ceder, and Martin Z Bazant. Intercalation dynamics in rechargeable battery materials: general theory and phase-transformation waves in lifepo 4. Electrochimica Acta, 53(26):7599-7613, 2008.

[82] Damian Burch and Martin Z Bazant. Size-dependent spinodal and miscibility gaps for intercalation in nanoparticles. Nano letters, 9(11):3795-3800, 2009.

[83] Manfred Martin. On the ionic conductivity of strongly acceptor doped, fluorite-type oxygen ion conductors. Journal of electroceramics, 17(2):765-773, 2006.

[84] M Meyer and N Nicoloso. Mc-study of the anomalous conductivity of fluorite-type solid oxide solutions. Berichte der Bunsen-Gesellschaft, 101(9):1393-1398, 1997.

[85] Marc C Kennedy and Anthony O'Hagan. Bayesian calibration of computer models. Journal of the Royal Statistical Society: Series B (Statistical Methodology), 63(3):425464, 2001.

[86] Walter R Gilks, Sylvia Richardson, and David Spiegelhalter. Markov chain Monte Carlo in practice. CRC press, 1995.

[87] David S Mebane, K Sham Bhat, Joel D Kress, Daniel J Fauth, McMahan L Gray, Andrew Lee, and David C Miller. Bayesian calibration of thermodynamic models 
for the uptake of co 2 in supported amine sorbents using ab initio priors. Physical Chemistry Chemical Physics, 15(12):4355-4366, 2013.

[88] Chong Gu and Grace Wahba. Smoothing spline anova with component-wise bayesian confidence intervals. Journal of Computational and Graphical Statistics, 2(1):97-117, 1993.

[89] Chong Gu. Smoothing spline ANOVA models, volume 297. Springer Science \& Business Media, 2013.

[90] Curtis B Storlie, Michael L Fugate, David M Higdon, Aparna V Huzurbazar, Elizabeth G Francois, and Douglas C McHugh. Methods for characterizing and comparing populations of shock wave curves. Technometrics, 55(4):436-449, 2013.

[91] K Sham Bhat, David S Mebane, Priyadarshi Mahapatra, and Curtis B Storlie. Upscaling uncertainty with dynamic discrepancy for a multi-scale carbon capture system. Journal of the American Statistical Association, 112(520):1453-1467, 2017.

[92] Brian J Reich, Curtis B Storlie, and Howard D Bondell. Variable selection in bayesian smoothing spline anova models: Application to deterministic computer codes. Technometrics, 51(2):110-120, 2009.

[93] Hidenori Yahiro, Yukari Eguchi, Koichi Eguchi, and Hiromichi Arai. Oxygen ion conductivity of the ceria-samarium oxide system with fluorite structure. Journal of applied electrochemistry, 18(4):527-531, 1988.

[94] R Gerhardt-Anderson and AS Nowick. Ionic conductivity of ceo2 with trivalent dopants of different ionic radii. Solid State Ionics, 5:547-550, 1981.

[95] Ding Rong Ou, Fei Ye, and Toshiyuki Mori. Defect clustering and local ordering in rare earth co-doped ceria. Physical Chemistry Chemical Physics, 13(20):9554-9560, 2011. 
[96] Masanobu Nakayama and Manfred Martin. First-principles study on defect chemistry and migration of oxide ions in ceria doped with rare-earth cations. Physical Chemistry Chemical Physics, 11(17):3241-3249, 2009.

[97] Fuyuki Shimojo, Tsuneyasu Okabe, Fumio Tachibana, Michisuke Kobayashi, and Hideo Okazaki. Molecular dynamics studies of yttria stabilized zirconia. i. structure and oxygen diffusion. Journal of the Physical Society of Japan, 61(8):2848-2857, 1992.

[98] Fuyuki Shimojo and Hideo Okazaki. Molecular dynamics studies of yttria stabilized zirconia. ii. microscopic mechanism of oxygen diffusion. Journal of the Physical Society of Japan, 61(11):4106-4118, 1992.

[99] BOH Grope, T Zacherle, M Nakayama, and M Martin. Oxygen ion conductivity of doped ceria: A kinetic monte carlo study. Solid State Ionics, 225:476-483, 2012.

[100] DS Park, J Griffith, AS Nowick, et al. Oxygen-ion conductivity and defect interactions in yttria-doped ceria. Solid State Ionics, 2(2):95-105, 1981.

[101] Steffen Grieshammer, Benjamin OH Grope, Julius Koettgen, and Manfred Martin. A combined dft+ $\mathrm{u}$ and monte carlo study on rare earth doped ceria. Physical Chemistry Chemical Physics, 16(21):9974-9986, 2014.

[102] Arif Ismail, Javier B Giorgi, and Tom K Woo. On the atomistic interactions that direct ion conductivity and defect segregation in the bulk and surface of samarium-doped ceria: A genetic algorithm study. The Journal of Physical Chemistry C, 116(1):704$713,2011$.

[103] K Binder and HL Frisch. Dynamics of surface enrichment: a theory based on the kawasaki spin-exchange model in the presence of a wall. Zeitschrift für Physik B Condensed Matter, 84(3):403-418, 1991. 
[104] Kurt Binder, Sanjay Puri, Subir K Das, and Jürgen Horbach. Phase separation in confined geometries. Journal of Statistical Physics, 138(1-3):51-84, 2010.

[105] Sanjay Puri and Kurt Binder. Surface-directed spinodal decomposition: phenomenology and numerical results. Physical Review A, 46(8):R4487, 1992.

[106] Sanjay Puri and Kurt Binder. Surface effects on spinodal decomposition in binary mixtures and the interplay with wetting phenomena. Physical Review E, 49(6):5359, 1994.

[107] Subir K Das, Jürgen Horbach, and Kurt Binder. Kinetics of phase separation in thin films: Lattice versus continuum models for solid binary mixtures. Physical Review E, 79(2):021602, 2009.

[108] BC Han, A Van der Ven, D Morgan, and G Ceder. Electrochemical modeling of intercalation processes with phase field models. Electrochimica Acta, 49(26):46914699, 2004.

[109] Eric A Lass, William C Johnson, and Gary J Shiflet. Correlation between calphad data and the cahn-hilliard gradient energy coefficient $\kappa$ and exploration into its composition dependence. Calphad, 30(1):42-52, 2006.

[110] KB Rundman and JE Hilliard. Early stages of spinodal decomposition in an aluminumzinc alloy. Acta metallurgica, 15(6):1025-1033, 1967.

[111] M Asta and JJ Hoyt. Thermodynamic properties of coherent interfaces in fcc-based ag-al alloys: a first-principles study. Acta materialia, 48(5):1089-1096, 2000.

[112] JJ Hoyt. Molecular dynamics study of equilibrium concentration profiles and the gradient energy coefficient in cu-pb nanodroplets. Physical Review B, 76(9):094102, 2007. 
[113] Donald R Franceschetti. Local thermodynamic formalism for space charge in ionic crystals. Solid State Ionics, 2(1):39-42, 1981.

[114] Natsuko Sakai, Haruo Kishimoto, Katsuhiko Yamaji, Teruhisa Horita, Manuel E Brito, and Harumi Yokokawa. Interface stability of perovskite cathodes and rare-earth doped ceria interlayer in sofcs. Journal of The Electrochemical Society, 154(12):B1331-B1337, 2007.

[115] Pei-Lin Chen and I-Wei Chen. Role of defect interaction in boundary mobility and cation diffusivity of ceo2. Journal of the American Ceramic Society, 77(9):2289-2297, 1994.

[116] Stefan Beschnitt, Tobias Zacherle, and Roger A. De Souza. Computational study of cation diffusion in ceria. The Journal of Physical Chemistry C, 119(49):27307-27315, 2015 .

[117] Steffen Grieshammer, Benjamin O. H. Grope, Julius Koettgen, and Manfred Martin. A combined $\mathrm{dft}+\mathrm{u}$ and monte carlo study on rare earth doped ceria. Phys. Chem. Chem. Phys., 16:9974-9986, 2014.

[118] Zhang Tianshu, Peter Hing, Haitao Huang, and J Kilner. Ionic conductivity in the $\mathrm{CeO}_{2}--\mathrm{Gd}_{2} \mathrm{O}_{3}$ system $(0.05 \leq \mathrm{gd} / \mathrm{ce} \leq 0.4)$ prepared by oxalate coprecipitation. Solid State Ionics, 148(3):567-573, 2002.

[119] JM Ralph, JA Kilner, and BCH Steele. Improving gd-doped ceria electrolytes for low temperature solid oxide fuel cells. In MRS Proceedings, volume 575, page 309. Cambridge Univ Press, 1999.

[120] Hugo J Avila-Paredes, Kwanghoon Choi, Chien-Ting Chen, and Sangtae Kim. Dopantconcentration dependence of grain-boundary conductivity in ceria: A space-charge analysis. Journal of Materials Chemistry, 19(27):4837-4842, 2009. 
[121] T Kudo and H Obayashi. Mixed electrical conduction in the fluorite-type ce1- x gd x o 2- x/2. Journal of The Electrochemical Society, 123(3):415-419, 1976.

[122] VV Kharton, FM Figueiredo, L Navarro, EN Naumovich, AV Kovalevsky, AA Yaremchenko, Ap P Viskup, AMFMB Carneiro, FMB Marques, and JR Frade. Ceria-based materials for solid oxide fuel cells. Journal of Materials Science, 36(5):1105-1117, 2001.

[123] Thomas J DiCiccio, Robert E Kass, Adrian Raftery, and Larry Wasserman. Computing bayes factors by combining simulation and asymptotic approximations. Journal of the American Statistical Association, 92(439):903-915, 1997. 